\title{
Nonlinear dynamics and breakup of free-surface flows
}

\author{
Jens Eggers \\ Universität Gesamthochschule Essen, Fachbereich Physik, 45117 Essen, Germany
}

\begin{abstract}
Surface-tension-driven flows and, in particular, their tendency to decay spontaneously into drops have long fascinated naturalists, the earliest systematic experiments dating back to the beginning of the 19th century. Linear stability theory governs the onset of breakup and was developed by Rayleigh, Plateau, and Maxwell. However, only recently has attention turned to the nonlinear behavior in the vicinity of the singular point where a drop separates. The increased attention is due to a number of recent and increasingly refined experiments, as well as to a host of technological applications, ranging from printing to mixing and fiber spinning. The description of drop separation becomes possible because jet motion turns out to be effectively governed by one-dimensional equations, which still contain most of the richness of the original dynamics. In addition, an attraction for physicists lies in the fact that the separation singularity is governed by universal scaling laws, which constitute an asymptotic solution of the Navier-Stokes equation before and after breakup. The Navier-Stokes equation is thus continued uniquely through the singularity. At high viscosities, a series of noise-driven instabilities has been observed, which are a nested superposition of singularities of the same universal form. At low viscosities, there is rich scaling behavior in addition to aesthetically pleasing breakup patterns driven by capillary waves. The author reviews the theoretical development of this field alongside recent experimental work, and outlines unsolved problems. [S0034-6861(97)00303-6]
\end{abstract}

\section{CONTENTS}

I. Introduction

II. Experiments
A. Jet
B. Dripping faucet
C. Liquid bridge

III. Simulations
A. Inviscid, irrotational flow
B. Stokes flow
C. Navier-Stokes simulations

IV. Small Perturbations
A. Linear stability
B. Spatial instability
C. Higher-order perturbative analysis

V. One-Dimensional Approximations
A. Radial expansion method
B. Averaging method: Cosserat equations
C. Basic properties and simulations
D. Inviscid theory and conservation laws

VI. Similarity Solutions and Breakup
A. Local similarity form
B. Before breakup
C. Stability and the influence of noise
D. After breakup

VII. Away From Breakup
A. High viscosity—threads
B. Low viscosity-cones
C. Satellite drops

VIII. Related Problems
A. Two-fluid systems
1. Stationary shapes
2. Breakup
B. Electrically driven jets
C. Polymeric liquids

IX. Outlook

Acknowledgments

References

865

869

869

872

873

874

875

876

877

878

878

882

883

885

886

888

889

892

894

894

895

897

900

904

904

907

911

914

914

915

917

919

922

924

925

926

\section{INTRODUCTION}

The formation of drops is a phenomenon ubiquitous in daily life, science, and technology. But although it is plain that drops generically result from the motion of free surfaces, it is not easy to predict the distribution of their sizes or to observe the intricate dynamics involved; see Fig. 1. Only the extremely short flash used by the photographer, Harold Edgerton, clearly reveals the formation of individual drops. Thus the subject has been far from exhausted after more than 300 years of scientific research, which in fact has gained considerable momentum only recently. On the one hand, the reason for this interest lies in the tremendous technological importance of drop formation in mixing, spraying, and chemical processing, which leads to applications such as ink-jet printing, fiber spinning, and silicon chip technology. On the other hand, the modern theory of nonlinear phenomena has created a new paradigm of self-similarity and scaling, which opened a new perspective on this classical problem.

The first mention of drop formation in the scientific literature is in a book by Mariotte (1686) on the motion of fluids. He notes that a stream of water flowing from a hole in the bottom of a container decays into drops. Like many authors after him, he assumes that gravity, or other external forces, are responsible for the process. A simple estimate shows, however, that uniform forces cannot lead to drop formation and that another force, which of course is surface tension, is responsible for the eventual breakoff of drops. For reasons of mass conservation, the rate at which the minimal cross section of a fluid filament decreases is proportional to the cross section itself, multiplied by an axial velocity gradient. As long as this gradient is finite, as is to be expected when only uniform forces are acting, the decrease of the minimum thickness will be at most exponential, leading to separation only in infinite time.

The basis for a more thorough understanding of drop formation was laid by Savart (1833), who very carefully investigated the decay of fluid jets. By illuminating the jet with sheets of light, he observed tiny undulations growing on a jet of water, as shown in Fig. 2. These 


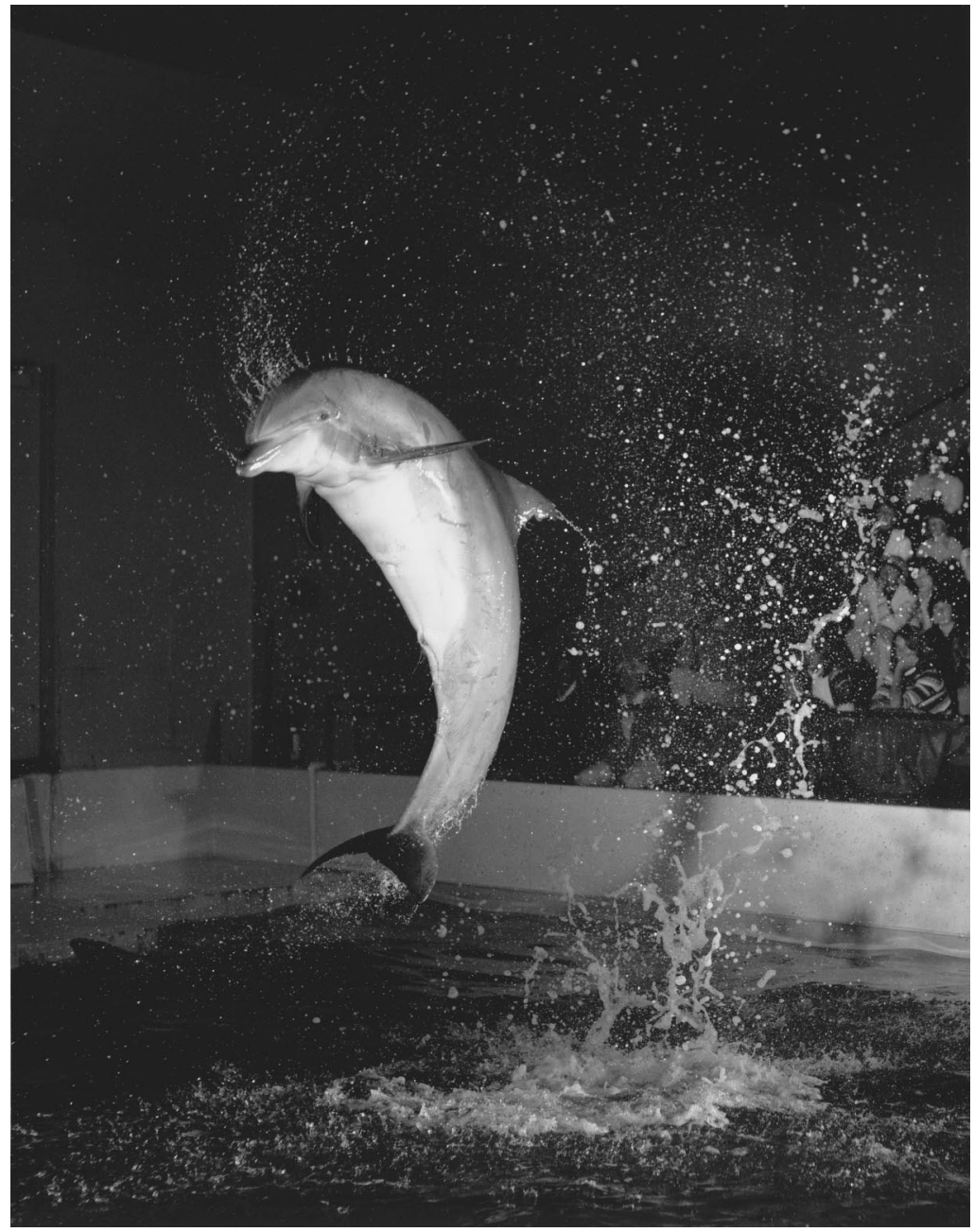

FIG. 1. A dolphin in the New England Aquarium in Boston, Massachusetts; Edgerton (1977). (C) The Harold E. Edgerton 1992 Trust, courtesy of Palm Press, Inc. undulations later grow large enough to break the jet. Savart's research showed that (i) breakup always occurs independent of the direction of gravity, the type of fluid, or the jet velocity and radius, and thus must be an intrinsic property of the fluid motion; (ii) the instability of the jet originates from tiny perturbations applied to the jet at the opening of the nozzle.

In spite of his fundamental insights, Savart did not recognize surface tension, which had been discovered some years earlier (de Laplace, 1805; Young, 1805) as the source of the instability. This discovery was left to (Plateau 1849), who showed that perturbations of long wavelength reduce the surface area and are thus favored by surface tension. On a level of quasistatic motion it would thus be desirable to collect all the fluid into one sphere, corresponding to the smallest surface area. Evidently, as shown in Fig. 1, this does not happen. It was Rayleigh (1879a,1879b) who noticed that surface tension has to work against inertia, which opposes fluid motion over long distances. By considering small sinusoidal perturbations on a fluid cylinder of radius $r$, Rayleigh found that there is an optimal wavelength, $\lambda_{R} \approx 9 r$, at which perturbations grow fastest, and which sets the typical size of drops. Analyzing data Savart had obtained almost 50 years earlier, Rayleigh was able to confirm his theory to within $3 \%$.
Accordingly, the time scale $t_{0}$ on which perturbations grow and eventually break the jet is given by a balance of surface tension and inertia, and thus

$$
t_{0}=\left(\frac{r^{3} \rho}{\gamma}\right)^{1 / 2},
$$

where $\rho$ is the density and $\gamma$ the coefficient of surface tension of the fluid. This tells us two important things: Substituting the values for the physical properties of water and $r=1 \mathrm{~mm}$, one finds that $t_{0}$ is $4 \mathrm{~ms}$, meaning that the last stages of pinching happen very fast, far below the time resolution of the eye. Secondly, as pinching progresses and $r$ gets smaller, the time scale becomes shorter and pinching precipitates to form a drop in finite time. At the pinch point, the radius of curvature goes to zero, and the small amount of fluid left in the pinch region is driven by increasingly strong forces. Thus the velocity goes to infinity, and the separation of a drop corresponds to a singularity of the equations of motion, in which the velocity and gradients of the local radius diverge.

Even in the case of an infinite-time singularity of the equations of hydrodynamics, the physical event of breaking may occur in finite time. That is, when the fluid thread has become sufficiently thin, it may break owing 


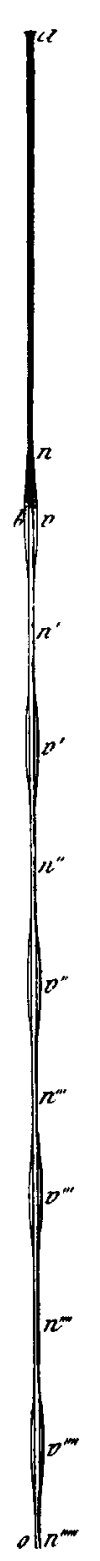

FIG. 2. Perturbations growing on a jet of water (Savart, 1833).

to microscopic effects that are outside the realm of hydrodynamics. The crucial distinction from the finite-time singularity, which results from surface tension, is that there is a chance to describe breaking in terms of continuum mechanics alone without resorting to microscopic notions. The description of such singularities will form a substantial part of this review.

For one hundred years after Rayleigh's original work, theoretical research focused on the extension of his results on linear stability. For example, Rayleigh (1892) himself considered a highly viscous fluid, but the general Navier-Stokes case was only treated in 1961 by Chandrasekhar. Tomotika (1935) took the surrounding fluid into account; Keller, Rubinow, and Tu (1973) looked at the growth of a progressive wave rather than a uniform perturbation of a cylinder. To illustrate the power of Rayleigh's ideas even in completely different fields we mention the application of linear stabilty to the breakup of nuclei (Brosa, Grossmann, and Müller, 1990), in which case the equivalent of a surface tension has to be calculated from quantum mechanics. Another example is the instability observed on pinched tubular vesicles (Bar-Ziv and Moses, 1994), where entropic forces drive the motion.

Meanwhile, experimental results had accumulated that probed the dynamics of free surfaces beyond the validity of linear theory. Early examples include Rayleigh's photographs of jets (1891), Worthington's study of splashes (1908), and Edgerton, Hauser, and Tucker's (1937) photographic sequences of dripping faucets. Experimental techniques have also become available more recently with sufficient resolution in space and time to look at the immediate vicinity of the point of breakup. Notable examples include the jet experiments of Rutland and Jameson (1970) as well as those of Goedde and Yuen (1970) for water jets and of Kowalewski (1996) for jets of high-viscosity fluids. A momentous paper by Peregrine, Shoker, and Symon (1990) not only helped to crystallize some of the theoretical ideas, but also contained the first high-resolution pictures of water falling from a faucet. For higher viscosities, corresponding pictures were taken by Shi, Brenner, and Nagel (1994).

By comparison, the development of computer codes that would permit the calculation of free-surface flows from first principles has been slow. Owing to the difficulties involved in implementing both moving boundaries and surface tension, resolution has not been possible anywhere near the experimentally attainable limit, even with present-day computers. An important exception is the highly damped case of the breakup of a viscous fluid in another, which recently led to a detailed comparison between experiment and numerical simulation (Tjahjadi, Stone, and Ottino, 1992).

Only gradually did the theoretical tools evolve that allowed for an analytical description of the nonlinear dynamics close to breakup. The first was developed in the theory of waves and often goes by the name of "lubrication theory" or "the shallow-water approximation" (Peregrine, 1972). It captures nonlinear effects in the limit of small depths compared with a typical wavelength. During the 1970s, lubrication approximations were developed for the corresponding axisymmetric problem, to study drop formation in ink-jet printers. This is of particular relevance since a jet does not break up uniformly, as predicted by linear theory, but rather into main drops and much smaller "satellite" drops. The satellite drops fundamentally limit the print quality attainable with this technology, as drops of different sizes are deflected differently by an electric field, which should direct the stream of droplets to a given position on the paper. Thus a fully nonlinear theory is needed to understand and to control satellite formation. The first dynamical equation, based on lubrication ideas, was introduced by Lee (1974) for the inviscid case. His nonlinear simulations indeed showed the formation of satellite drops. But it took two decades until systematic approximations of the Navier-Stokes equation were found that included viscosity (Bechtel, Forest, and Lin, 1992; Eggers and Dupont, 1994). 
Another important concept, which allows for the description of nonlinear effects, is that of self-similarity (Barenblatt, 1996), which arises naturally in problems that lack a typical length scale. In the case of a singularity, the length scale of the solution will depend on time, reaching arbitrarily small values in the process. Thus self-similarity here means that the solution, observed at different times, can be mapped onto itself by a rescaling of the axes. In the context of flows with surface tension, self-similarity was introduced by Keller and Miksis (1983). Kadanoff and his collaborators (Constantin et al., 1993; Bertozzi et al.., 1994) have looked at singularities in a Hele-Shaw cell, which is the twodimensional analogue of the present problem, as a simple model for singularity formation. They ingeniously combined lubrication ideas and self-similarity to arrive at a detailed description of the pinchoff of a bubble of fluid.

In the wake of this success, Eggers (1993) and Eggers and Dupont (1994) applied the same idea to the threedimensional case. As spelled out first by Peregrine et al. (1990), the dynamics near breakup are independent of the particular setup such as jet decay, a dripping faucet, or even the complicated spraying shown in Fig. 1, but rather are characteristic of the nonlinear properties of the equations of motion. As the motion near a point of breakup gets faster, only fluid very close to that point is able to follow, making the breakup localized both in space and time. Thus one expects the motion to become independent of initial conditions, and the type of experiment becomes irrelevant to the study of the singular motion. This brings about two crucial simplifications: (i) in a local description around the point of breakup, the motion becomes "universal," thus reducing the number of relevant parameters. The only parameter upon which the motion near the singularity still depends is the length

$$
\ell_{\nu}=\frac{\nu^{2} \rho}{\gamma}
$$

which characterizes the internal properties of the fluid (Peregrine et al., 1990; Eggers and Dupont, 1994); (ii) an asymptotic analysis of the equations of motion reveals that the motion close to the singularity is self-similar, with the radius shrinking at a faster rate than the longitudinal extension of the singularity. Near the pinch point, almost cylindrical necks develop, making the motion effectively one-dimensional close to the singularity.

Using these ideas, a local solution of the NavierStokes equation was found, which contained no free parameters (Eggers, 1993). To select a specific prediction of this theory, the minimum radius of a fluid thread at a given time $\Delta t$ away from breakup found to be

$$
h_{\min }=0.03 \frac{\gamma}{\rho \nu} \Delta t
$$

The surface profiles calculated from theory have been compared quantitatively and confirmed by experiment (Kowalewski, 1996). The columnar structure of the fluid neck allows for a stability analysis of the flow close to the breaking point, and is modeled closely on Rayleigh's analysis of a liquid cylinder (Brenner, Shi, and Nagel, 1994; see also Brenner, Lister, and Stone, 1996). As the neck becomes sufficiently thin, it is prone to a finiteamplitude instability, which may be driven by thermal noise. This causes secondary necks to grow on the primary neck, which again have a self-similar form. The corresponding complicated structure of nested singularities has also been observed experimentally (Shi, Brenner, and Nagel, 1994).

At the same time stability analysis indicates that cylindrical symmetry is not just a matter of convenience, but rather a generic property near breakup. Rayleigh's analysis tells us that any azimuthal variation results in only a relative increase in surface area and is thus unfavorable. The universality and stability of the solution near breakup therefore lead to answers of a much greater generality than could be hoped for by investigating individual geometries and initial conditions. At the same time, the singular motion is the natural starting point for the calculation of nonlinear properties away from breakup, which controls phenomena such as satellite formation. Another advantage of universality is that only one particular initial condition needs to be investigated to construct a unique continuation of the NavierStokes equation to times after the singularity (Eggers, 1995a). This establishes that breaking is described by continuum mechanics alone, without resorting to a microscopic description, as long as observations are restricted to macroscopic scales.

The scope of this review is limited mostly to the dynamics in the immediate vicinity of the point of breakup. This is motivated by the expectation that pinching is universal under quite general circumstances, even if the motion farther away from the singularity is more complicated. In the nonasymptotic regime, our focus is on the axisymmetric case of a jet with or without gravity. This excludes many important examples of nonlinear free-surface motion, such as drop oscillations, the dynamics of fluid sheets, and in particular the vast field of surface waves.

We begin with an overview of the experimental basis of the subject. Here and in the rest of this review, we confine ourselves to cylindrical symmetry. In the case of free surfaces, this is representative of the majority of experimental work in the physics literature. But it excludes important effects like bending (Entov and Yarin, 1984; Yarin, 1993), branching (Lin and Webb, 1994), and spraying (Yang, 1992) of jets. There is also substantial work on splashes, i.e., the impact of drops on liquid (O $\widetilde{g}$ uz and Prosperetti, 1990) or on solid surfaces (Yarin and Weiss, 1995) surfaces. Mixing processes can also not be expected to respect cylindrical symmetry. The outline of experimental work in the second section is complemented by a review of numerical work in the third section. As indicated above, numerical simulations of the full hydrodynamic equations are only slowly catching up with the resolution possible in experiments. On the other hand, important information on the velocity field is not available experimentally. This and the superior variability of simulations, for example, in the choice of 
fluid parameters and of initial conditions, is bound to make simulations an important source of information.

In the fourth section we give a detailed account of linear stability theory, which is the classical approach to the problem, but which remains an area of research to the present day. Some nonlinear effects can be included in perturbation theory, but the expansion quickly breaks down near pinching.

The groundwork for the description of nonlinear effects is laid by the development of one-dimensional models. We spell out two different approaches to the problem and explain some of the properties of the resulting models in Sec. V. In Sec. VI we study in detail the universal self-similar solution leading up to breakup. The solution can be continued uniquely to a new solution valid after breakup, which now consists of two parts. The nonlinear stability theory of the asymptotic solution explains the complicated structure seen in the presence of noise.

Section VII explores the dynamics away from the asymptotic regime, but where nonlinear effects are still dominant. The area best understood is the case of highly viscous jets, in which the pinching has not yet become sufficiently fast for inertial effects to become important. In the opposite limit of very low viscosity, the smoothing effect of viscosity is missing, and gradients of the flow field become large at a finite time away from breakup. This makes the problem a hard one, and the understanding of this regime is only in its early stages. However, this subject is bound to remain an interesting and frequently studied topic for the years to come, since low viscosity fluids like water are the most common. From a theoretical point of view, the understanding of the singularities of the Euler equation is one of the major unsolved problems in hydrodynamics, and fluid pinching serves as a particularly simple model system. To conclude Sec. VII, we describe some research on satellite formation and present a numerical simulation of the stationary state of a decaying jet.

So far we have dealt only with free surfaces, with surface tension being the only driving force. We relieve this restriction in the final section, where we explore some examples of related topics. First we look at two-fluid systems, which are particularly important for the theory of mixing and the hydrodynamics of emulsions. An asymptotic theory for breakup in the presence of an outer fluid has not yet been developed. Electric or magnetic fields represent another possible external driving force. They force the fluid into sharp tips, where the fields are strong, out of which tiny jets are ejected. This allows for the production of very fine sprays. In chemical processing, macromolecules are often present in solution. They result in non-Newtonian properties of the fluid, to which we give a brief introduction in the context of free-surface flows.

\section{EXPERIMENTS}

Historically, research on drop formation was motivated mostly by engineering applications, hence the three most common experimental setups, which are described in more detail below: (1) Jets are produced when a fluid leaves a nozzle at high speeds; (2) slow dripping under gravity has been used for the measurement of surface tension; and (3) liquid bridges are used to suspend fluid in the absence of gravity. For a review on drop formation in the context of engineering applications of spraying, see Walzel (1988). Early work focused either on the early stages of drop formation, characterized by the growth of linear disturbances, or on the size and number of the resulting drops. Either aspect of drop formation is relatively easy to observe, but is highly dependent on the experimental setup and on parameters like nozzle diameter or jet speed.

Only slowly, as experimental techniques became available for observing the actual evolution of the flow during drop formation, did common features emerge from the seemingly disparate results of individual experiments. The last stages of the evolution are dominated by the properties of the pinch singularity, which is the same for all cases. This idea was first enunciated clearly by Peregrine et al. (1990). The appearance of the motion depends only on the scale of observation $\ell_{\text {obs }}$ relative to the size of the internal length $\ell_{\nu}$ [see Eq. (2)] of the fluid. If $\ell_{\text {obs }} / \ell_{\nu}$ is large, which is typical for flows of low viscosity like water, the shapes near the pinch point are cones attached to a spherical shell. After breakup, as the fluid neck recoils, capillary waves are excited. If on the other hand $\ell_{\text {obs }} / \ell_{\nu}$ is small, as for fluids of high viscosity like glycerol, long and skinny threads are observed, which rapidly contract into tiny drops after breakup. At the highest viscosities, the threads tend to break at random places. It is from this universality that much of the physical interest of the subject of drop formation derives, and we try to emphasize common features in discussing different kinds of experiments.

\section{A. Jet}

By far the most widely used experimental setup in the study of drop formation is that of a jet of fluid leaving an orifice at high speeds. The earliest jet experiments were performed with fluid being driven out of holes near the bottom of a container (Bidone, 1823). The focus of the early research was on the shape of jets produced by orifices of different forms. It was Savart (1833) who distinctly noticed the inevitability of a decay into drops and carefully investigated the laws governing it. By deliberately disturbing the jet periodically at the nozzle, he produced disturbances on the surface of the jet with the same frequency. Many other 19th-century researchers repeated these experiments, notably Hagen (1849), Magnus (1855), and Rayleigh (1879b,1882). Both Plateau (1873) and Rayleigh were able to perform some quantitative tests of their theories, but without photography it was impossible to record the shapes of jets in detail. Photographic methods were introduced by Rayleigh (1891), but these observations were only qualitative in nature. The first quantitative experiments were 


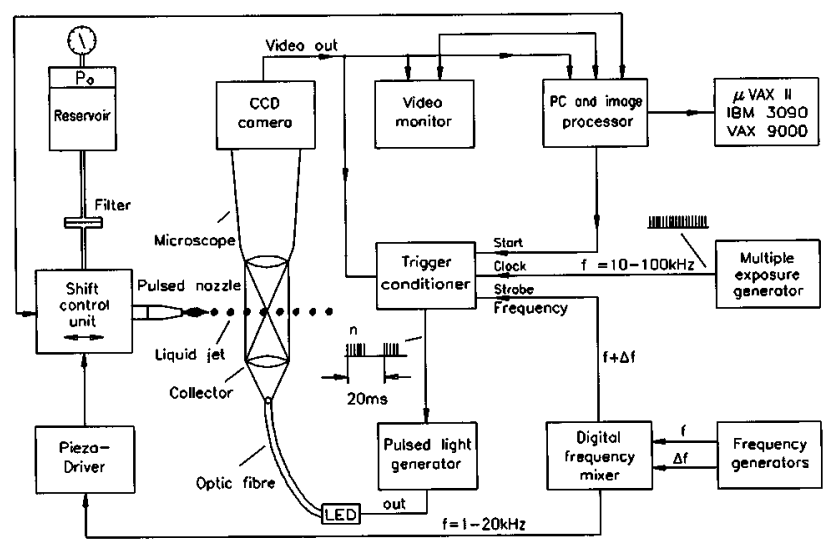

FIG. 3. Experimental setup for the stroboscopical observation of a decaying jet as developed by Becker, Hiller, and Kowalewski (1991).

those of Haenlein (1931), Donelly and Glaberson (1966), and Goedde and Yuen (1970). Their main concern was to test the linear theories of Rayleigh (1879a, 1892) and Chandrasekhar (1961) for different viscosities. Goedde and Yuen also recorded shapes characterizing the nonlinear behavior near breakup. Experiments focusing on the measurement of satellites were performed by Rutland and Jameson (1970), Chaudhary and Maxworthy (1980a,1980b), and Vasallo and Ashgriz (1991). Becker, Hiller, and Kowalewski $(1991,1994)$ studied the nonlinear oscillations of drops produced in the breakup process. Their experimental setup was used by Kowalewski (1996) to record surface shapes near breakup with a spatial and temporal resolution far superior to previous work. We shall describe this experiment in some detail, since it demonstrates the degree of sophistication the experimental technique has acquired over the last few decades.

Figure 3 gives an overall flow chart of the apparatus (Becker, Hiller, and Kowalewski, 1991). Fluid is forced out of a nozzle directed vertically downward, so the axis of symmetry is preserved. Typical jet speeds are of the order of $\mathrm{m} / \mathrm{s}$, so the air drag can be neglected. On the other hand, the breakup takes place far from the nozzle (approximately 1000 jet diameters), so the entire region of interest can be regarded as being in free fall. Nozzle diameters vary between $1 / 10 \mathrm{~mm}$ and $2 \mathrm{~mm}$. In an antechamber of the nozzle a piezoceramic transducer produces pressure oscillations, which translate into sinusoidal disturbances of the jet speed at the nozzle exit. As a result, the jet breaks into drops in a perfectly periodic fashion. This is an advantage of this experimental setup, as it is amenable to observations by the stroboscopic method pioneered by Rayleigh (1882).

To this end the region of interest is illuminated with the light of a pulsed light-emitting diode (LED), the typical duration of the flash being $0.2 \mu$ s. The jet is then viewed through a microscope and images are recorded with a charge-couple device (CCD) camera. The optics is set up in bright field illumination, exposing parallel light to the camera, so that the image is bright. If a piece of fluid is in the path of the light, the light is diffracted away from the camera's view, so it appears black, apart from a bright center, where light passes straight through the fluid. This allows for spatial resolution of about 1 $\mu \mathrm{m}$. To make observations of a given stage in jet decay, one arranges for the flash to illuminate the jet with the same frequency with which it is excited. If the light pulse is ensured to be in a fixed phase with the current driving the jet modulator, a stationary image is seen. By changing the phase, a different time within the evolution of one period is illuminated. The whole process is so stable and reproducible that a time resolution of $10 \mu \mathrm{s}$ is reached. By scanning an entire range of phases, movies of the breakup process with this time resolution can be produced, some examples of which are presented in Sec. VI.

We now discuss the dimensionless parameters controlling jet decay. We shall assume that the driving is purely sinusoidal, so that the speed at the nozzle is

$$
v_{\text {nozzle }}=v_{j}+\epsilon\left(\frac{\gamma}{\rho r_{0}}\right)^{1 / 2} \sin (2 \pi f t) \text {. }
$$

Here $v_{j}$ is the speed of the jet and $r_{0}$ its unperturbed radius. The dimensionless perturbation amplitude $\epsilon$ is multiplied by the capillary velocity $u_{0}=\left(\gamma /\left(\rho r_{0}\right)\right)^{1 / 2}$. By properly adjusting the driving frequency $f$, we can choose a wavelength $\lambda=v_{j} / f$. In typical experimental situations the frequency is several $\mathrm{kHz}$.

The parameter most significant to jet decay is the reduced wave number

$$
x=2 \pi r_{0} / \lambda \text {. }
$$

If $x<1$, the jet is unstable to the corresponding perturbations, and at small viscosities, $x_{R}=0.697$ is the most "dangerous"- or the Rayleigh mode for which perturbations grow fastest. For $x>1$ irregular breakup is observed, as the jet responds to tiny random perturbations with components $x<1$. The temporal perturbation is translated into space by convection with velocity $v_{j}$. The time scale on which surface perturbations grow is $t_{0}=\left(\rho r_{0}^{3} / \gamma\right)^{1 / 2}$, since surface-tension forces will be balanced by inertia, at least at low viscosities. Thus the Weber number

$$
\beta^{2}=\rho r_{0} v_{j}^{2} / \gamma
$$

measures how much a disturbance can grow from one swell to the next. Typical values of $\beta$ are $O(100)$. The last parameter is the Reynolds number ${ }^{1}$

$$
\operatorname{Re}=u_{0} r_{0} / \nu=\left(r_{0} / \ell_{\nu}\right)^{1 / 2} \text {, }
$$

\footnotetext{
${ }^{1}$ It should be noted that the nomenclature in Eq. (7) is open to criticism, since at high viscosities the velocity scale is no longer set by surface tension and initial radius alone, but also depends on viscosity. A better name, used for example in McCarthy and Molloy (1974), is "Ohnesorge number." An Ohnesorge number conventionally is a number that depends on geometry and fluid properties alone. However, since "Reynolds number" is so much more likely to carry a meaning for physicists, we decided to be cavalier about these subtleties.
} 

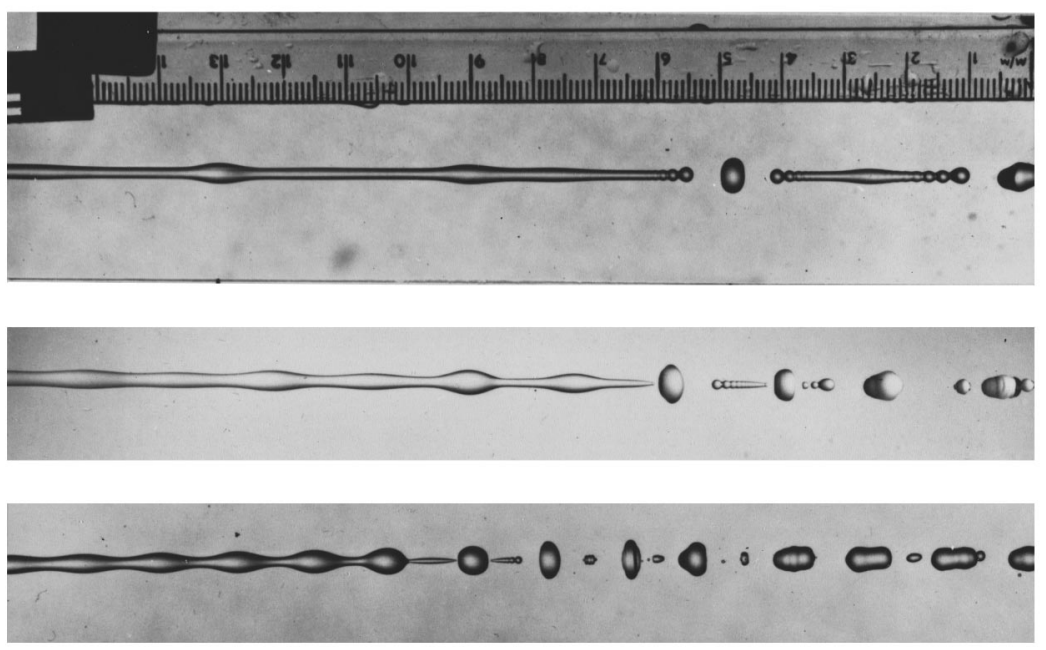

FIG. 4. Photographs of a decaying jet (Rutland and Jameson, 1971) for three different frequencies of excitation. The bottom picture corresponds to $x=0.683$, which is close to the Rayleigh mode. At longer wavelengths secondary swellings develop (middle picture, $x=0.25$ ), which cause the jet to break up at twice the frequency of excitation. At the longest wavelength (top picture, $x=0.075$ ) main and secondary swellings have become virtually indistinguishable. Reprinted with permission of Cambridge University Press. constructed from the jet radius $r_{0}$ and the capillary velocity $u_{0}$, divided by the kinematic viscosity $\nu=\eta / \rho$. It measures the damping effects of viscosity on the motion caused by surface tension. For water and a jet diameter of $1 \mathrm{~mm}, \mathrm{Re} \approx 200$, but technologically relevant fluids cover a wide range of different viscosities. For example, in the case of glycerol the Reynolds number is reduced to 0.5 , and by mixing water and glycerol a wide range of Reynolds numbers can be explored.

So, in the case of purely sinusoidal driving, there are four dimensionless parameters governing jet decay: the driving amplitude $\epsilon$, the reduced wave number $x$, the Weber number $\beta^{2}$, and the Reynolds number Re. The range of possible dynamics in this large parameter space has never been fully explored, so we shall focus on the dependence on the two most important parameters, the reduced wave number and the Reynolds number.

Figure 4 shows typical pictures of a decaying jet of water for three different wavelengths. The bottom picture is for the mode of maximum instability. It is easily found by tuning the frequency, since it makes the breakup point move closest to the nozzle. This situation is the least sensitive to noise, since all other frequencies have lower growth rates and therefore decay relative to the Rayleigh mode. Up to one wavelength from the point where a drop first separates, the disturbances look fairly sinusoidal. (There are significant higher-order corrections, though, which will be discussed in Sec. IV.) But the last neck pinches off almost simultaneously at both ends, causing significant deviations from linear, sinusoidal growth. This final, localized pinching produces characteristic forms, namely, a sharp conical tip attached to a flat cap. In recoiling, the tip excites capillary waves on its surface, which give it the appearance of a string of pearls. While the tip is still recoiling, it breaks on its rear side and starts recoiling on the other side as well. Thus a small satellite drop is produced, which is a remnant of the neck. In general it will receive momentum from the recoiling process and therefore has a velocity slightly different from the main drops. This will make it merge either with the preceding or the following drop a few wavelengths downstream.
If the wavelength is long enough, the growth rate of the second harmonic will be larger than that of the primary disturbance. Since higher harmonics are always excited at the nozzle or through the nonlinear interaction, a swell develops in the middle between the drops. If it has a chance to grow sufficiently large, the jet will break in this $\lambda / 2$ mode and produce drops and satellites at twice the fundamental frequency of excitation. This is shown in the middle picture of Fig. 4 for a driving frequency that is a factor of 0.36 below the frequency corresponding to the Rayleigh mode. The appearance of such a "double stream" of droplets thus depends sensitively on the amplitude of the second harmonic produced by the driving. Plateau (1857) used a cello to excite the jet, and always found a double stream at half the frequency of the Rayleigh mode. Later Rayleigh (1882) showed that this was due to harmonics inconveniently produced by a musical instrument. Using tuning forks instead, he still observed breakup with the principal frequency at a third of the Rayleigh frequency. For even longer wavelengths (top picture), the satellite drop becomes substantial, owing to the much longer neck. This causes the recoil patterns to be even more pronounced, since there is more time for capillary waves to develop. As a result, the satellite drop is subject to very complicated secondary breakings.

Decreasing the Reynolds number significantly, for example, by increasing the viscosity using a glycerol-water mixture, causes the breakup process to change substantially. After the initial sinusoidal growth, a region develops where almost spherical drops are connected by thin threads of almost constant thickness, which take quite a long time to break (see Fig. 5). In general, the thread will still break close to the swells. If the viscosity is increased further, the threads become so tenuous before they break at the end, that they break at several places in between, in what seems to be a random breakup process.

Jet experiments are particularly useful for studying the universal motion near breakup with extremely high precision, as we shall see in Sec. VI. On the other hand, it is hard to design an experiment to make the full evo- 


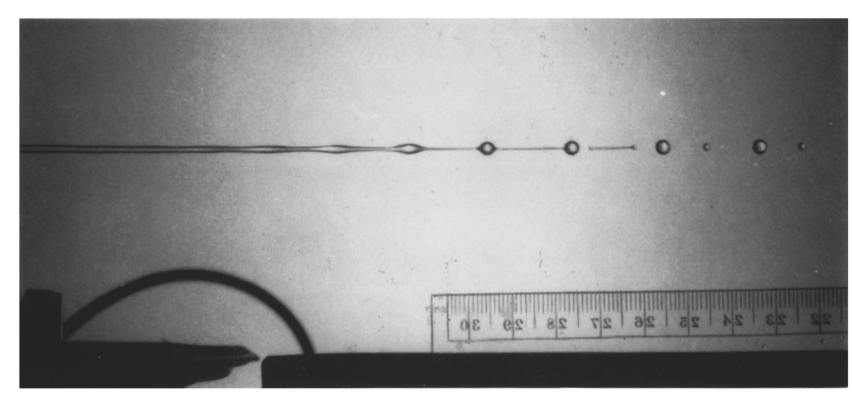

FIG. 5. A photograph of a viscous jet (Donnelly and Glaberson, 1966) for a reduced wave number of $x=0.268$ and a Reynolds number of 2 . A thread connecting two main drops has just broken at the ends and is contracting into a satellite drop. Reprinted with permission of the Royal Society.

lution of the jet leading up to breakup completely reproducible. This is because the driving is never purely sinusoidal, but contains harmonics that depend on the nozzle design and that significantly influence jet evolution. Thus it is hard to make exact quantitative comparisons with numerical simulations, comparisons that are needed to validate numerical codes. The great merit of the two other experiments to be described in the following two subsections is that they are both simple, and all of the dynamics are completely reproducible.

\section{B. Dripping faucet}

This experimental setup involves the opposite limit to that of a jet: Fluid is released very slowly from a nozzle, so that at first surface-tension forces are in balance with gravitational forces. One can easily reach a limit where the flow plays no role and the hanging drop goes through a sequence of equilibrium shapes. These sequences have been studied very carefully by Worthington (1881). The theory of their stability was begun by Plateau (1873) and Maxwell (1875) and is summarized by Michael and Williams (1976). Instability will set in sooner or later as the drop becomes heavier and gravity overcomes surface tension. All these static aspects can only depend on the dimensionless number

$$
\mathrm{Bo}=\rho g r_{0}^{2} / \gamma,
$$

which is called the Bond number and expresses the ratio of gravitational forces to surface-tension forces.

After the initial instability, the fluid begins to fall and eventually a drop separates. This familiar phenomenon has been the subject of some early work (Guthrie, 1864; Tate, 1864). Tate measured the drop weight $W$ and noticed a proportionality to the nozzle radius. Rayleigh (1899) analyzed the problem in terms of dimensionless groups and arrived at the simple empirical relation $W=3.8 \gamma r_{0}$. To produce an accurate method for the measurement of surface tension, Harkins and Brown (1919) performed a careful experimental study of the relation between the dimensionless drop weight $W /\left(\gamma r_{0}\right)$ and the Bond number. They found a complicated functional form, which deviated considerably from the proportionality proposed by Tate. In fact, it was pointed out by Hauser et al. (1936), as well as Edgerton, Hauser, and Tucker (1937), that it was unlikely that a simple theoretical derivation for such a relation existed. In fact, their high-speed motion pictures revealed a very complicated structure produced by the neck between the main drop and the nozzle. As in the jet experiments, this structure resulted in the production of one or more satellite drops. They also noticed a significant dependence on viscosity, with a long and thin neck forming at high viscosities. The beauty of the experiment lies in the fact that the only other dimensionless parameter needed to specify the entire evolution is the Reynolds number $\operatorname{Re}=\left(r_{0} \gamma / \nu^{2} \rho\right)^{1 / 2}$.

But it was only the pioneering work of Peregrine et al. (1990) that focused on the dynamics immediately before and after the bifurcation. The whole sequence of events contains no free parameters, which makes it an ideal testing ground for theory. Later, this work was extended to higher viscosities by Shi, Brenner, and Nagel (1994). Figure 6 shows a sequence of single flash photographs of a water drop. Shortly after the lower part of the hanging drop begins to fall, it produces a neck on which surface tension acts, making it thinner. At a certain stage, pinching sets in and a drop separates. Again the pinch point is very localized and the shapes remarkably resemble Fig. 4. Recoil produces capillary waves, but before the tip can completely retreat the neck breaks at the other end, the flat part now being on top of the cone. The whole sequence of pictures is completely reproducible. Qualitatively, this process is quite similar for different Bond numbers, but the size of the main drop and the length of the neck increase with Bond number. At high viscosities, a transition to long and thin necks is observed. A particular example is shown in Fig. 7, with a fluid 100 times more viscous than water. Note the emergence of a tiny thread coming out of the neck just above the drop. Just as in the jet experiments, at the highest viscosities threads form which are thin enough to be prone to random breakup.

In addition to the single flash photographs described above, Zhang and Basaran (1995) and Brenner et al. (1997) produced time-resolved motion pictures of the bifurcation. From those, the temporal dependence of neck radius and neck length could be measured. Zhang and Basaran not only varied the radius of the nozzle and the viscosity of the fluid, but also the flow rate, to obtain a detailed "phase diagram" of main drop and satellite sizes, final neck lengths, etc. The dependence of drop size on the flow rate has also been measured and compared with a simple one-dimensional theory by Wilson (1988). Singular properties like the temporal evolution of the radius of the neck close to pinchoff were found to be insensitive to the flow rate (Zhang and Basaran, 1995). On the other hand, viscosity had a profound influence, not only on the final length of the neck, but also on the time dependence of the radius. This is in accord with the expectation that singular properties depend only on the internal length $\ell_{\nu}$. To measure the neck radius well below the time scale $t_{0}=\left(r_{0}^{3} \rho / \gamma\right)^{1 / 2}$, which is 


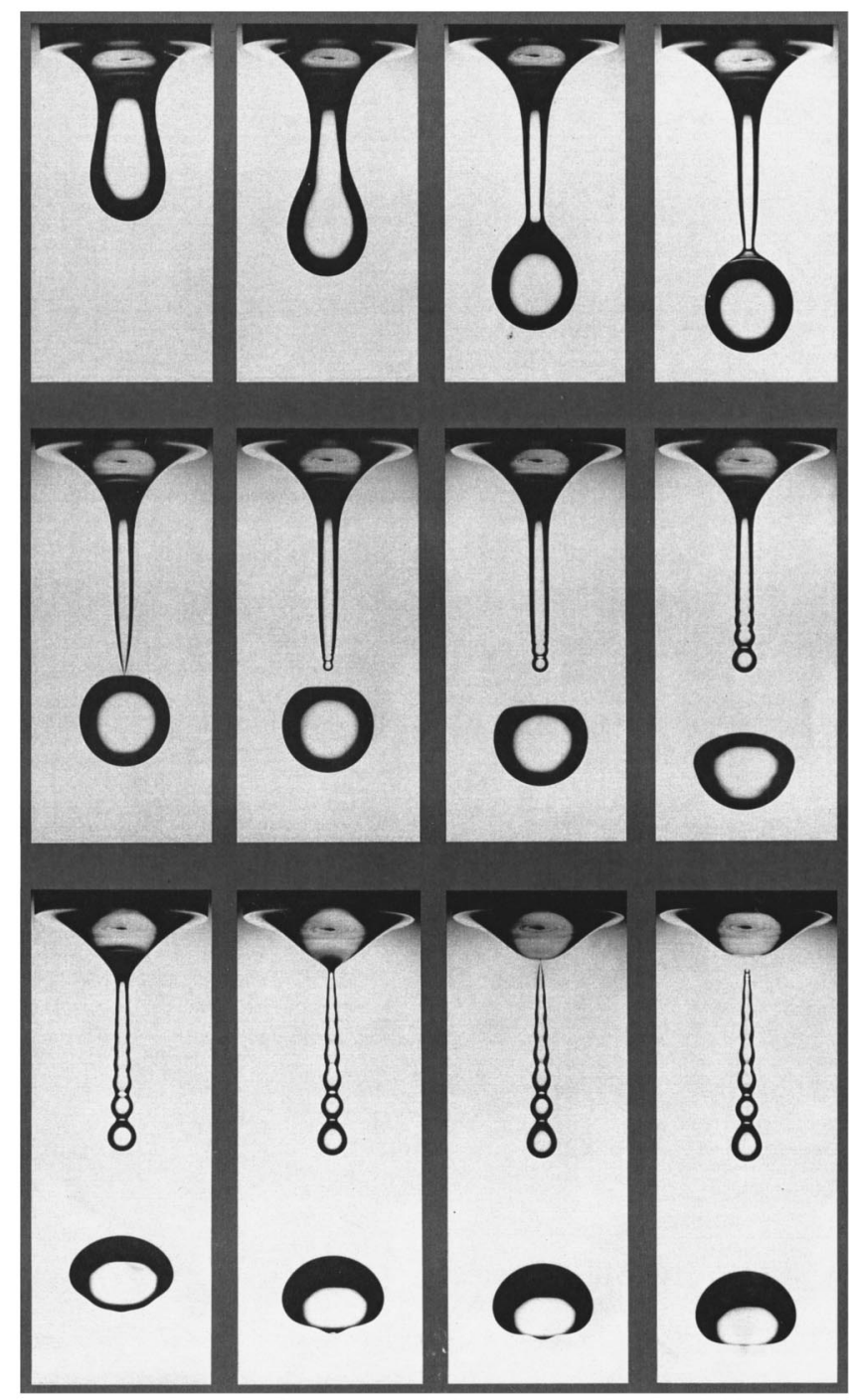

FIG. 6. A sequence of pictures of a water drop falling from a circular plate $1.25 \mathrm{~cm}$ in diameter (Shi, Brenner, and Nagel, 1994). The total time elapsed during the whole sequence is about 0.1 s. Reprinted with permission. (C) American Association for the Advancement of Science.

of the order of $10 \mathrm{~ms}$ for typical experiments with water, Brenner et al. (1997) took high-speed motion pictures with a time resolution of $20 \mu$ s. Thus a detailed analysis of scaling properties at low viscosity was possible, as explained in more detail in Sec. VII B.

\section{Liquid bridge}

The last experimental setup to be described here is that of a piece of fluid between two disks; see Fig. 8. The fluid is held in place by surface tension, which is possible only for very small bridge sizes if gravity is present. Therefore experiments are designed either to be performed in space (Meseguer, Sanz, and Lopez, 1986) or with an outer fluid of the same density in a so-called Plateau tank (Spiegelberg, Gaudet, and McKinley, 1994). It is this setup which Plateau $(1843,1849)$ used for

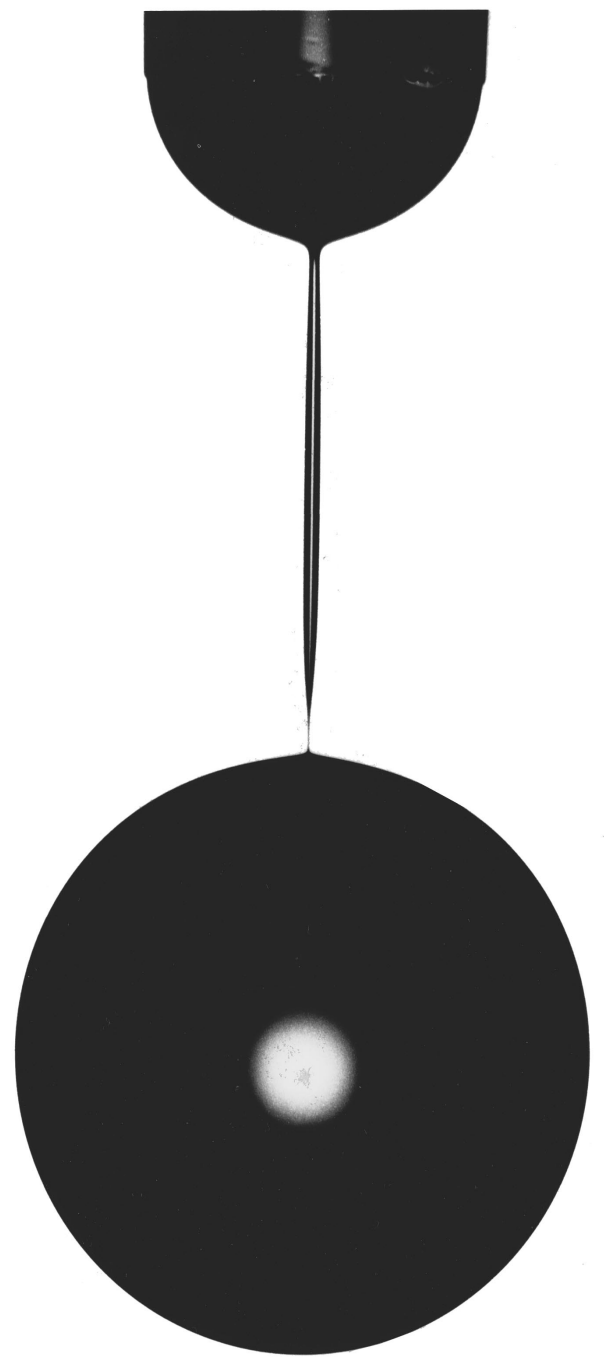

FIG. 7. A drop of a glycerol and water mixture, 100 times as viscous as water, falling from a nozzle $1.5 \mathrm{~mm}$ in diameter. As opposed to the case of water, a long neck is produced (Shi, Brenner, and Nagel, 1994). Reprinted with permission. (C) American Association for the Advancement of Science.

his detailed experimental investigation of the stability of liquid cylinders. The disadvantage of the Plateau tank is that the inner fluid can no longer be tested in isolation, and the dynamics of the outer fluid has to be taken into account as well. The effect of the outer fluid on the dynamics can be partly eliminated by working with a fluid of very high viscosity, much larger than that of the outer fluid, so that the viscosity of the outer fluid can be neglected. At the same time the motion is slowed down so as to make the inertia of the outer fluid irrelevant as well.

Most of the experimental work in the liquid-bridge configuration is motivated less by an interest in breakup, than by the desire to hold a free fluid drop in place. It is used to measure rheological properties of liquids and to investigate crystal growth in the absence of gravity. Nevertheless, this configuration is highly appealing for our purposes as well, for its simplicity and for the ease 

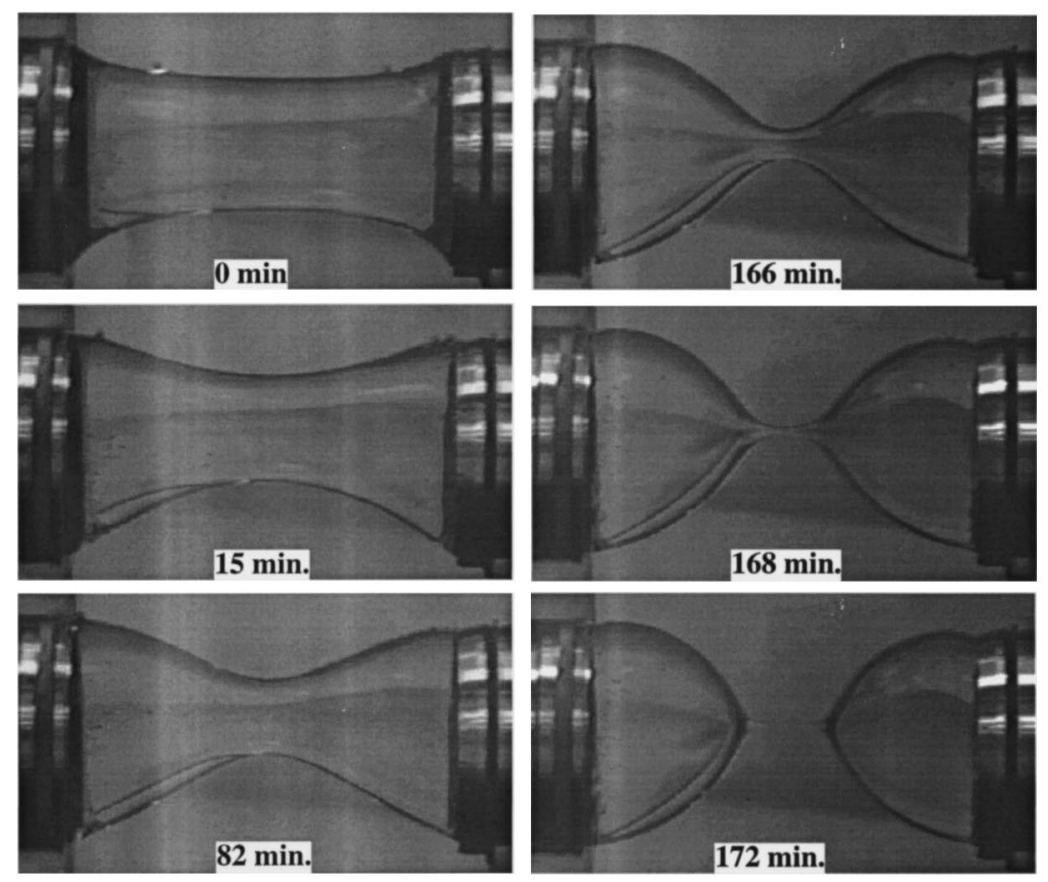

FIG. 8. Liquid-bridge evolution starting from an unstable configuration. The disk diameter is $3.8 \mathrm{~cm}$, the Reynolds number is $3.7 \times 10^{-3}$. The outer fluid, which eliminates buoyancy forces, has a viscosity approximately 1000 times smaller than the inner fluid. (Spiegelberg, Gaudet, and McKinley, 1994).
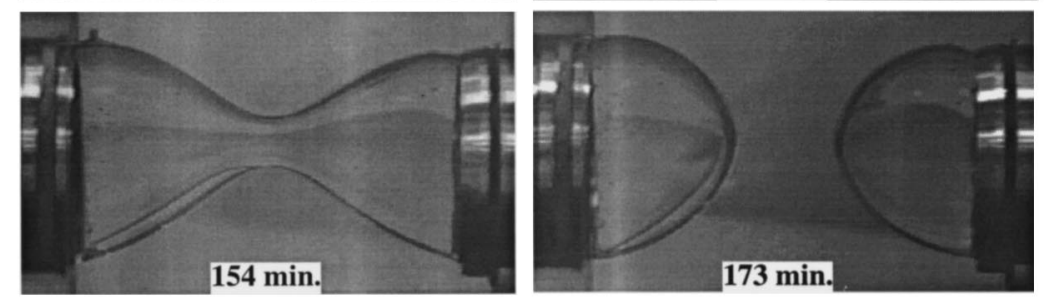

with which the experimental parameters can be controlled. To begin with, the problem of static stability of the bridge becomes purely geometrical: it depends only on the radii of the disks and the volume of the fluid. This stability has been investigated theoretically in a number of circumstances (Gillette and Dyson, 1971; DaRiva, 1981).

If one wants to observe breaking, the bridge has to be made unstable. This can be achieved either by sucking fluid from the bridge (Meseguer and Sanz, 1985) or by pulling the disks apart (Spiegelberg, Gaudet, and McKinley, 1994). The latter method is illustrated in Fig. 8. The initial state was that of a cylinder with aspect ratio $\Lambda=r_{0} / L=0.77$, where $2 L$ is the distance between the disks. Over a period of a few minutes, it was pulled apart to an aspect ratio of $\Lambda=1.58$, which is an unstable configuration. The figure shows the collapse after the disks stopped moving. As expected for the extremely low Reynolds number of $3.7 \times 10^{-3}$, a thin thread forms before the bridge finally breaks.

\section{SIMULATIONS}

In many areas of fluid mechanics, flow simulations have become standard procedure. If the Reynolds number is not too high, carefully executed simulations can virtually replace experiments, since the validity of the equations of motion is not a source of concern. In the presence of a free surface, however, the situation is dif- ferent. The flow geometry is essentially determined by the evolution itself or may change its topology altogether due to breakup events. Thus computations need to be tailored to each initial condition. If breakup occurs, the validity of continuum mechanics, underlying the equations of motion, is itself called into question. This concern must be addressed separately through a more careful study of the singularities involved, or through complementing simulations of the underlying molecular dynamics (Greenspan, 1993; Koplik and Banavar, 1993).

Free-surface flows are also very sensitive to the formation of cusp singularities (Joseph et al., 1991) even in seemingly innocuous flow situations. It seems as if surface tension should make the surface more regular, thus simplifying simulations. But instead it offers very little resistance to the formation of singularities (Jeong and Moffatt, 1992) and makes the system more sensitive to noise and prone to numerical instabilities, whose nonlinear origins are poorly understood. Indeed, surface tension introduces a complicated coupling between the flow that advances the interface and the interface's shape, which through the Young-Laplace equation determines the pressure driving the fluid.

For that reason, so-called boundary integral formulations are very attractive, since they involve only information about the surface of the fluid. They are possible whenever the flow is governed by a linear equation, for which the Green's function is known. This is the case for 


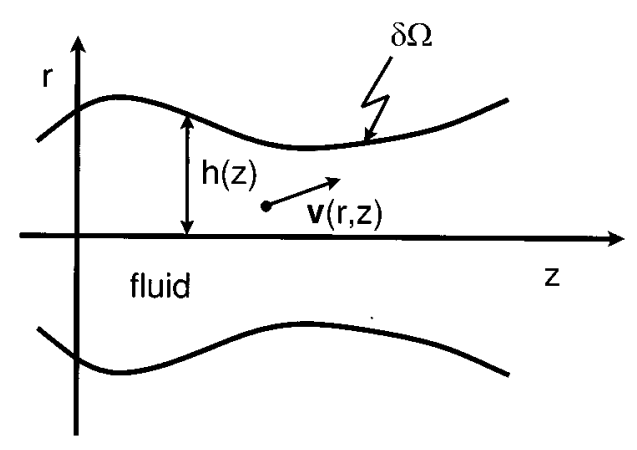

FIG. 9. Sketch of a typical flow geometry. Rotational symmetry around the $z$ axis is assumed. The velocity field inside the fluid is $\mathbf{v}(r, z)=v_{z}(r, z) \mathbf{e}_{z}+v_{r}(r, z) \mathbf{e}_{r}$.

inviscid, irrotational flow and highly viscous flow governed by the Stokes equation. Then the only information needed apart from the position of the interface is its velocity. Thus one is relieved from calculating the subtle interplay of internal motion and the boundary shape, since all the information is contained on the boundary. In the more general case of Navier-Stokes flow, the interior and the boundary have to be dealt with separately. Both the surface tracking and the flow computations are highly nontrivial problems in themselves and are now coupled in a complicated fashion. Such computations have been performed only fairly recently and are not sufficiently accurate to resolve the universal behavior close to the singularity. Boundary integral methods are more accurate, but neglect either viscous or inertial forces, both of which become important asymptotically. Nevertheless, simulations are an indispensable tool for predicting the nonuniversal dynamics away from breakup. One hopes that numerical codes will soon become sufficiently reliable to yield an alternative to experiments. A useful overview on computational methods of free-surface flows is provided by Tsai and Yue (1996), who draw most of their examples from problems related to free-surface waves.

\section{A. Inviscid, irrotational flow}

We first turn to the problem of a low-viscosity drop with a free surface (see Fig. 9). Most studies assume axisymmetry, so the boundary is given by a curve in the $r-z$ plane, but this is not essential to our discussion. If the velocity field is irrotational initially, and viscosity can be neglected, it will remain so for the rest of its evolution (Landau and Lifshitz, 1984a). Thus the velocity potential $\phi$,

$$
\mathbf{v}(r, z)=\boldsymbol{\nabla} \phi(r, z)
$$

obeys the Laplace equation

$$
\Delta \phi=0 \text {. }
$$

The evolution of $\phi$ follows from the Bernoulli equation

$$
\partial_{t} \phi+\mathbf{v}^{2} / 2+p / \rho=0
$$

where $p$ is the pressure. On the boundary, and in absence of viscous forces, the pressure is given by the Young-Laplace formula, hence

$$
\partial_{t} \phi=-\mathbf{v}^{2} / 2-\frac{\gamma}{\rho}\left(\frac{1}{R_{1}}+\frac{1}{R_{2}}\right) \text { on } \partial \Omega,
$$

where $R_{1}, R_{2}$ are the principal radii of curvature.

If $\mathbf{x}(\xi, t)$ is the position of the surface as a function of some marker (or grid point) $\xi$, the surface moves according to

$$
\partial_{t} \mathbf{x}(\xi, t)=\left.\mathbf{v}\right|_{\partial \Omega} .
$$

Note that the definition of $\xi$ as a surface marker makes the left-hand side a convective time derivative. The potential can be written as a function of the marker, as well, according to

$$
\phi(\xi, t)=\phi(\mathbf{x}(\xi, t), t),
$$

and the resulting Lagrangian evolution equation is

$$
\partial_{t} \phi(\xi, t)=\mathbf{v}^{2} / 2-\frac{\gamma}{\rho} \kappa \text { on } \partial \Omega .
$$

Here we abbreviated $\left(1 / R_{1}+1 / R_{2}\right)$, which is twice the mean curvature, to $\kappa$. Thus Eqs. (12) and (13) give the evolution for both the surface and the value of the potential on it, provided the velocity $\mathbf{v}$ on the surface is known. Decomposed into tangential and normal components, $\mathbf{v}$ can be written as

$$
\mathbf{v}=\left(\partial_{s} \phi\right) \mathbf{n}+\left(\partial_{n} \phi\right) \mathbf{t},
$$

where $\mathbf{n}$ and $\mathbf{t}$ are unit vectors normal and tangential to the surface, respectively. The tangential derivative $\partial_{s} \phi$ can be evaluated from the knowledge of $\phi$ on the surface alone, but to compute $\partial_{n} \phi$ one must bring the Laplace equation into play. That is, given Eq. (10), it follows from Green's second theorem that

$$
2 \pi \phi(\mathbf{r})=\text { P.V. } \int_{\partial \Omega}\left(\phi\left(\mathbf{r}^{\prime}\right) \partial_{n} \frac{1}{\left|\mathbf{r}-\mathbf{r}^{\prime}\right|}-\frac{\partial_{n^{\prime}} \phi\left(\mathbf{r}^{\prime}\right)}{\left|\mathbf{r}-\mathbf{r}^{\prime}\right|}\right) d^{2} s^{\prime},
$$

where both $\mathbf{r}$ and $\mathbf{r}^{\prime}$ lie on the surface. This is an integral equation that can be solved for $\partial_{n} \phi$, once $\phi$ is known on $\partial \Omega$.

The procedure outlined was developed by LonguetHiggins and Cokelet (1976) for the study of water waves in two dimensions. It has been adapted and used for the study of drops by Dommermuth and Yue (1987) and Schulkes (1994a, 1994b). Most of the computing time is spent on solving the integral equation (15), which is a matrix equation in discrete form. If the number of grid points is $N$, then the effort in computing the matrix elements is $\sim N^{2}$, and to invert the matrix $\sim N^{3}$. For $N \approx 100$, a typical value for current computations, most of the time is spent evaluating the matrix coefficients, which contain elliptic integrals. Clearly, if much higher resolution is to be attained, matrix inversion becomes the limiting factor. Therefore other authors have used a method originally developed for vortex simulations by Baker, Meiron, and Orszag (1980). The idea is first to calculate a distribution of dipoles $\mu$ on the surface, 
which produce the potential $\phi$. This can be done efficiently by iteration. The dipole distribution is then used to calculate the normal component of the velocity field, which is an $N^{2}$ process. For the present problem, this procedure has been adopted by $\mathrm{O} \widetilde{\mathrm{g}} \mathrm{uz}$ and Prosperetti (1990) and Mansour and Lundgren (1990).

To formulate the system comprised by Eqs. (12)-(15) in discrete numerical form, a number of different procedures were adopted, some of which are compared by Pelekasis, Tsamopoulos, and Manolis (1992). Usually the position of the interface $\mathbf{x}$ and the potential $\phi$ is taken at a discrete set of nodes $i, 1 \leqslant i \leqslant N$. Using suitable interpolation formulas, one evaluates the curvature at the interface and the integral in Eq. (15). An explicit Runge-Kutta step is then used to advance the position of the interface $\mathbf{x}_{i}$ and the potential $\phi_{i}$. However, a problem that appears, regardless of the numerical implementation, is nonlinear instability on the scale of the grid spacing, giving the interface a saw-tooth appearance. The origin of this instability has not yet been found. Moore (1983), investigating a model problem, has shown that such short-wavelength instabilities may be a result of spatial discretization. But since the instability was reported in all work on the problem, using many different numerical techniques, there is a distinct possibility that the evolution equations (10), (12), and (13) do not possess regular solutions, but rather have unphysical singularities before breakup occurs. One has to bear in mind that the underlying equations are inviscid, so there is no natural way for momentum to be diffused, and catastrophically sharp gradients can occur, just as in the three-dimensional Euler equation with large-scale driving (Majda, 1991). This analogy is of course a very rough one, since singularities in the three-dimensional Euler equation come about through the growth of vorticity, which is constrained to be zero in the present case of irrotational flow.

A number of different procedures have been proposed to get rid of the instability. Grid points were redistributed after every time step to ensure equal grid spacing (Dommermuth and Yue, 1987; O $\widetilde{g}$ uz and Prosperetti, 1990; Schulkes, 1994a). This step removes shortwavelength components and results in some numerical energy dissipation (Schulkes, 1994a). Other possibilities are the inclusion of numerical diffusion (O $\widetilde{\mathrm{g}} \mathrm{uz}$ and Prosperetti, 1990; Pelekasis, Tsamopoulos, and Manolis, 1992), or simply filtering of the short-wavelength components (Dold, 1992).

Only a few papers have focused on drop formation, namely those of Mansour and Lundgren (1990), who simulated a liquid cylinder with periodic boundary conditions; Spangler, Hilbing, and Heister (1995), who included the effect of a surrounding gas; and Schulkes (1994b), who considered a dripping faucet. The spatially uniform liquid cylinder is only a rough approximation of the steady state of a decaying liquid jet, but the simulations qualitatively produce the correct features. In particular, Mansour and Lundgren predict satellite drops at all wave numbers, whose volumes agree well with experiment.

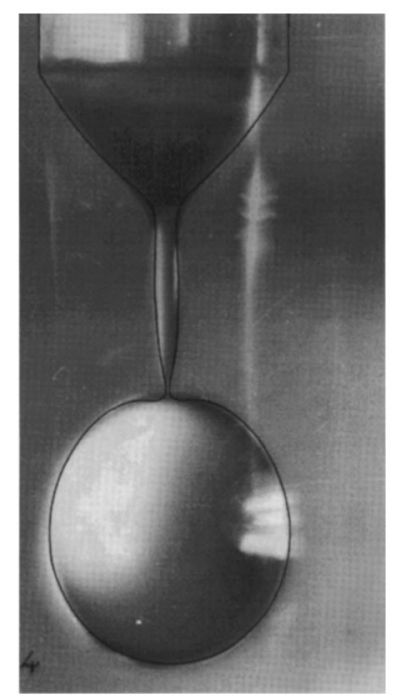

FIG. 10. The shape of a drop of water falling from a nozzle at the first bifurcation. The Bond number is $\mathrm{Bo}=1.02$ and the Reynolds number $\mathrm{Re}=452$. The comparison with theory (solid line) is taken from Schulkes (1994b). Reprinted with permission of Cambridge University Press.

Schulkes (1994b) directly compares his simulation with the experiment by Peregrine et al. (1990) and finds good agreement, except in the immediate neighborhood of the bifurcation point (see Fig. 10). Instead of forming an almost flat interface at the side of the drop, the profile turns over in the inviscid simulation. This seems to be a universal feature of this approximation, as a similar overturning is observed for the initial condition considered by Mansour and Lundgren. In experiments, a very large, but still finite, slope is observed. The steepening of the interface has been the subject of a recent experimental and numerical study (Brenner et al., 1997).

Overturning thus seems to be an artifact of the inviscid theory, since the viscosity does become important as the interface becomes steeper, as will be discussed in more detail in Sec. VII.B. The inviscid approximation is thus invalidated even on scales much larger than $\ell_{\nu}$. However, overturning is observed after breaking, when the small remnant of the neck on the drop side recoils. The momentum it acquires is large enough to produce a dent on the flat surface of the drop. In experimental pictures, which usually show a projection perpendicular to the axis of symmetry, it turns up as a perfectly straight interface, as seen in the sixth frame of Fig. 6. It would be worthwhile to take pictures at an angle as well, to further investigate the dented region.

\section{B. Stokes flow}

The other case that can be treated by boundary integral methods is that of a highly viscous fluid, described by the Stokes equation

$$
\eta \Delta \mathbf{v}=\nabla p .
$$


Here inertial terms have been dropped, so the fluid density does not appear. For Eq. (16) Green's functions are known (Ladyzhenskaya, 1969), so that an integral equation for the interfacial velocity can be derived in the same vein as in the inviscid case. This was first accomplished by Youngren and Acrivos (1975) for a gas bubble in a very viscous fluid. The method has been generalized by Rallison and Acrivos (1978) to the case of drops of arbitrary viscosity in another viscous fluid. However, the early numerical work on viscous drops was limited to the calculation of stationary shapes (Rallison, 1984).

Only some years later did Stone and Leal (1989a, 1989b) develop codes of sufficient accuracy to study the dynamics of extended drops. Because of the ample viscous damping, this problem is much less sensitive to perturbations, and no numerical instabilities have been reported. Most of the work concerns a drop of fluid in another fluid, which is quiescent at infinity. However, the viscosity of the outer fluid can be taken to be zero, so the drop is isolated.

Some pictures of extended drops breaking up are contained in Stone and Leal (1989b). The shape of the interface near breakup and the velocity field is studied in some detail. But only Tjahjadi, Stone, and Ottino (1992) demonstrated the full power of viscous boundary integral methods by performing a quantitative comparison with experiments on viscous drops in extensional flows. This study showed extraordinary agreement between simulation and experiment, which continued through a number of breakups (see Fig. 11), and revealed an astonishingly fine structure of satellite drops. Breakup was simulated by cutting the interface and smoothing the ends once some critical radius was reached. As the fluid moves faster and faster near breakup, the Stokes approximation eventually will break down, but on the scales observed it seems to remain remarkably good. A similar Stokes code has recently been applied to a stretching liquid bridge (Gaudet, McKinley, and Stone, 1996). The results are in excellent agreement with experimental data, and in addition supply a wealth of information about the dynamics of the bridge at different viscosity ratios and stretching rates.

\section{Navier-Stokes simulations}

The boundary integral methods described in the first subsection are valid only for very low or very high viscosity. As we we are going to see in Sec. VII, either approximation breaks down close to the pinch point, where viscous or inertial effects become important, at least in the case that the outer fluid can be neglected. In those cases, the full Navier-Stokes equation has to be solved in the fluid domain, subject to a singular forcing at the boundary. Even in a fixed domain this is not a simple problem, so it is not surprising that Navier-Stokes simulations in the nonlinear regime are few. Still, results from first-principles calculations are indispensable, as they provide the full information on the flow field, which is not available from experiment. Johnson, Marschall,

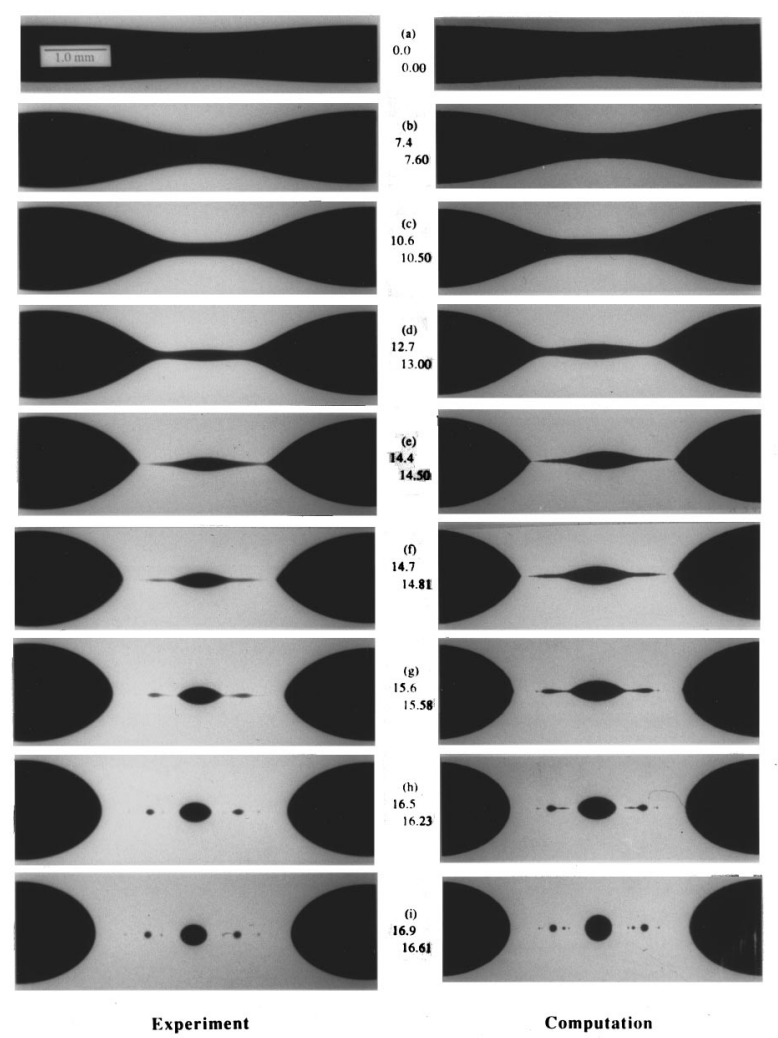

FIG. 11. Time evolution of a highly extended fluid suspended in another fluid. The viscosity ratio is 0.067 , and the dimensionless wave number is 0.5 . The times the snapshots were taken are shown in the middle (Tjahjadi, Stone,and Ottino, 1992). Reprinted with permission of Cambridge University Press.

and Esdorn (1985) and Marschall (1985) provide some experimental measurements of the flow field, but the region near the point of breakup is particularly hard to visualize, so the flow field is not known here.

The interior of the flow is described by the NavierStokes equation

$$
\partial_{t} \mathbf{v}+\mathbf{v}(\nabla \mathbf{v})=-\frac{1}{\rho} \nabla p+\nu \Delta \mathbf{v}
$$

for incompressible flow

$$
\nabla \mathbf{v}=0 \text {. }
$$

Here $\mathbf{v}(\mathbf{r}, t)$ is the three-dimensional velocity field and $p(\mathbf{r}, t)$ the pressure. On the free boundary, pressure and viscous forces are balanced by capillary forces:

$$
\boldsymbol{\sigma} \cdot \mathbf{n}=-\left.\gamma \kappa \mathbf{n}\right|_{\partial \Omega} .
$$

In this formula, $\boldsymbol{\sigma}$ is the stress tensor and $\mathbf{n}$ the normal vector pointing out of the fluid. With the velocity known, the interface is moved according to Eq. (12).

The main technical problem, as compared to other Navier-Stokes simulations, involves the coupling to a movable interface and the treatment of a varying computational domain. The earliest work is that by Fromm (1984), who uses a square grid with a refined grid superimposed on it to track the surface. The paper focuses on 
the specific problem of ink-jet technology and does not study pinching systematically. In the work by Shokoohi and Elrod (1987, 1990), the computational domain is mapped onto a cylinder with radius 1 , making surface tracking superfluous. The price one has to pay is that the equations in the interior get very complicated. In particular, the earlier paper (Shokoohi and Elrod, 1987) contains a number of surface profiles for different initial disturbances at a fixed Reynolds number of $\mathrm{Re}=50$.

The technique most appropriate for the problem is probably the one adopted by Keunings (1986), which uses a deformable grid to accommodate the moving interface. A similar technique was recently used in the most extensive study of breakup to date (Ashgriz and Mashayek, 1995). Here the fluid is bounded by a height function $h(z, t)$; see Fig. 9. Thus one is limited to situations in which the profile does not overturn. The computational domain is divided into quadrangles in the $r-$ $z$ plane, four of which constitute a column in the radial direction. On each of the quadrangles, four shape functions are defined, into which the flow field is expanded. Using a Galerkin method, one can derive a discrete set of equations for the amplitudes of the velocity field and the boundary points $h_{i}$. To follow the motion of the interface, the mesh points are allowed to move in the radial direction. This is done in such away as to ensure mass conservation in each element. From this the free surface is reconstructed. Simulations are reported over a wide range of Reynolds numbers up to $\mathrm{Re}=200$, which corresponds to a water jet of $1 \mathrm{~mm}$ in diameter. No numerical instabilities are reported even at the highest Reynolds number.

Simulations were performed with periodic boundary conditions in the axial direction and for initial sinusoidal disturbances of different wave numbers. The results were tested against the predictions of linearized theory (Rayleigh, 1879a; Chandrasekhar, 1961), which are discussed in greater detail in the next section. The overall agreement with predicted growth rates is good. The largest deviations occur for the highest Reynolds number, for which a maximum error of $10 \%$ is reported. Particularly interesting is a sequence of pictures testing the nonlinear evolution leading to breakup for a variety of wave numbers and Reynolds numbers. Although the wave number affects the overall shape, the appearance of the interface near the point of breakup is very similar for different wave numbers. However, viscosity affects the breakup significantly: just as seen in experiment, the interface looks like a cone attached to a steep front for low viscosities and gets increasingly flat for higher viscosities. We shall study the interfacial shape in greater detail when we compare it with the results of onedimensional models (see Sec. V). One very important point to notice right away is that overturning of the profile does not occur, even at the highest Reynolds numbers, which are comparable with experimental ones with water.

Almost all the work described so far deals with the problem up to the point of first breakup. To continue through the singularity, some ad hoc prescription for a continuation has to be invented for each particular case, as was done in Tjahjadi, Stone, and Ottino (1992) or Schulkes (1994b). Apart from the theoretical questions involved, it would be desirable to develop a general phenomenological scheme to describe breakup and merging in an arbitrary geometry. Instead of tracking the interface with surface markers ("front tracking"), this is most easily accomplished by describing the interface by a scalar function $C(\mathbf{r}, t)$, which is defined everywhere ("front capturing"). This function is 0 in one fluid and 1 in the other. The crossover region represents the interface, which is maintained to have some finite thickness by numerical diffusion. The function $C$ is advanced everywhere according to

$$
\partial_{t} C+\mathbf{v}(\nabla C)=0 .
$$

Obviously this description does not rely on specific assumptions about the connectivity of the fluid domains. On a "microscopic" scale set by numerical diffusion, a thin sliver of fluid is dissolved and thus broken. Conversely, if two pieces of fluid come sufficiently close, they are joined. The most widely used variant of this idea is the "volume-of-fluid method" developed by Hirt and Nichols (1981), which is designed to conserve volume exactly. Surface tension is included in two recent papers by Lafaurie et al. (1994), and by Richards, Lenhoff, and Beris (1994) by distributing surface-tension forces continuously across the interface. The former group emphasizes collision and merging of drops, the latter studies breakup of jets. The computational domain, on which the Navier-Stokes solver operates, is stationary. Figure 12, showing two drops colliding at high speeds and their subsequent breakup, nicely illustrates the generality of this method.

On the other hand, the resolution available near breakup is not very high, so the singularity cannot be studied in detail. Lafaurie et al. (1994) also report some numerical instabilities at large Reynolds numbers. So despite some remarkable features, the development of general-purpose codes capable of faithfully resolving breakup in some detail remains a challenge for the future.

As a last possibility, we mention the work of Becker, Hiller, and Kowalewski (1994). It is concerned with large-amplitude droplet oscillations. The Navier-Stokes equation is here analyzed by expanding the velocity field into a set of eigenmodes of the linear problem. This method is very efficient when surface deformations are not too large, so that they are representable by a reasonable number of eigenmodes. However, one expects it to become inefficient close to rupture, where typical flow fields are dissimilar to eigenmodes of the linear problem.

\section{SMALL PERTURBATIONS}

\section{A. Linear stability}

For a piece of fluid to break up, it must be unstable against surface tension forces at some point during its evolution. If the fluid is at rest initially, or the time scale 

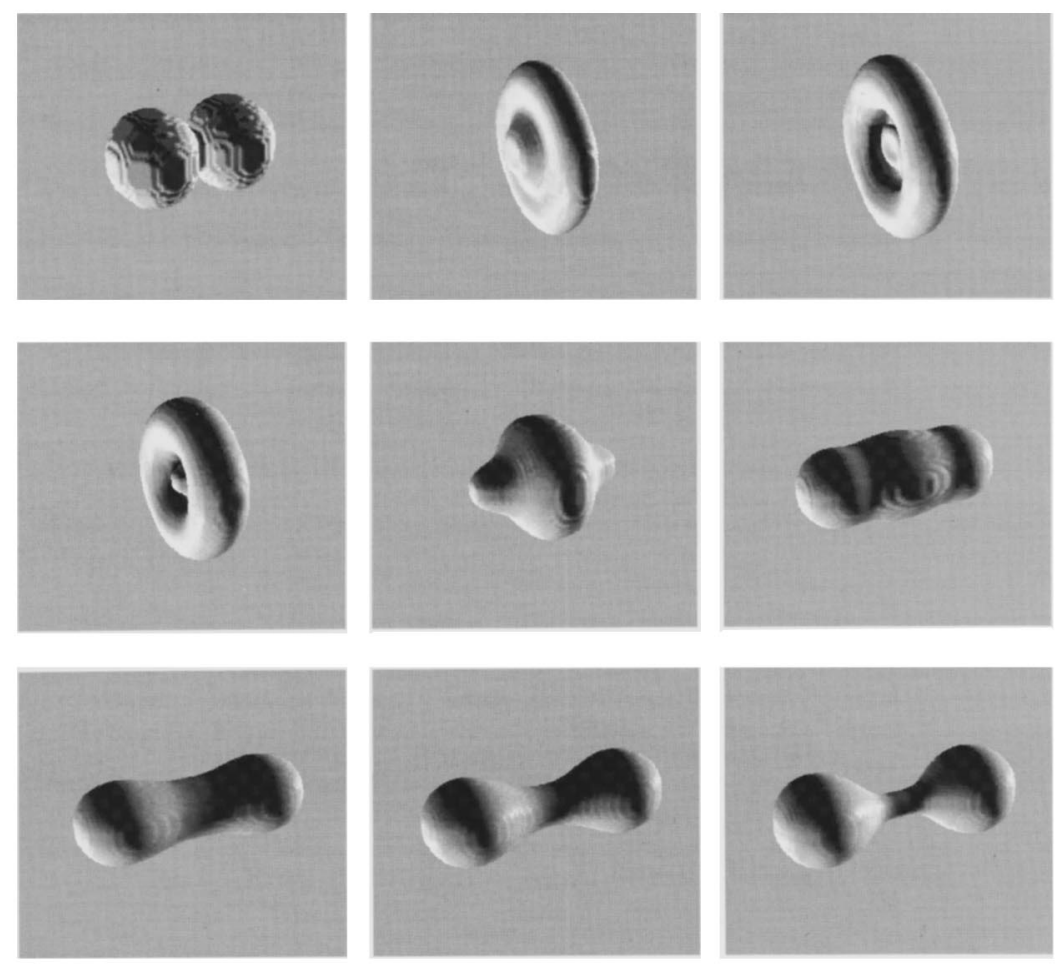

FIG. 12. Frontal collision of two drops at Weber number $\beta^{2}=20$ and Reynolds number $\operatorname{Re}$ $=250$, based on the relative velocity of the drops (Gueyffier and Zaleski, 1997). First a toroidal structure is formed, which then collapses and forms a cylinder. This cylinder breaks up as required by the Rayleigh instability.

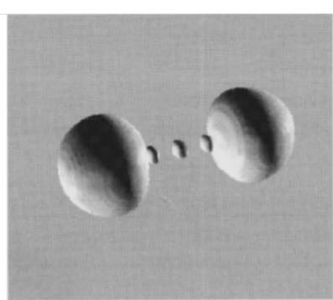

of the surface-tension-induced motion is much shorter than other time scales, then the problem of stability is purely geometrical; that is, instability means that the surface area can be reduced by an infinitesimal surface deformation.

The classical example studied by Plateau (1873) and Rayleigh (1896) is that of an infinitely long cylinder of radius $r_{0}$. To a good approximation such a fluid cylinder will be produced by a jet emanating from a nozzle at high speed. Now one considers a sinusoidal perturbation of wavelength $\lambda$ on the cylinder. For example, any random perturbation on the jet may be decomposed into a linear superposition of such Fourier modes. In a linear approximation all the modes evolve independently, and one may consider just a single sinusoidal perturbation. In most practical applications, however, perturbations of a given wavelength are produced at the nozzle and are convected down the jet. As argued in Sec. II, for large Weber numbers $\beta^{2}=\rho r_{0} v_{j}^{2} / \gamma$ this results in periodic disturbances, which are practically uniform in space.

In cylindrical coordinates $r, \varphi, z$ the surface shape can be written as $r(\varphi, z)$. It is convenient to measure the axial coordinate $z$ and deviations from the cylindrical shape $r(\varphi, z)=r_{0}$ in units of $r_{0}$ :

$$
\zeta=z / r_{0}
$$

and

$$
r(\varphi, z) / r_{0}=1+\eta(\varphi, \zeta) .
$$

In addition to the sinusoidal perturbation of the radius, we also allow for small departures from the circular cross section. This is taken care of by an additional azimuthal dependence of the initial perturbation:

$$
\begin{aligned}
& \eta(\varphi, \zeta)=\epsilon \cos (n \varphi) \cos (x \zeta), \\
& x=k r_{0} .
\end{aligned}
$$

The dynamical constraint is that the volume

$$
\begin{aligned}
V / r_{0}^{3} & =\frac{1}{2} \int_{0 \leqslant \zeta \leqslant 2 \pi / x}(1+\eta)^{2} d \varphi d \zeta \\
& =\frac{2 \pi^{2}}{x} \begin{cases}1+\epsilon^{2} / 2 & n=0 \\
1+\epsilon^{2} / 4 & n=1,2, \ldots\end{cases}
\end{aligned}
$$

per wavelength is kept constant as $\epsilon$ evolves in time. This means $\eta$ must also contain another contribution which ensures conservation of mass, and thus

$$
\eta(\varphi, \zeta)=\epsilon \cos (n \varphi) \cos (x \zeta)-\epsilon^{2}\left(1+\delta_{n 0}\right) / 8+O\left(\epsilon^{4}\right)
$$

On the other hand, the surface area is 


$$
\begin{aligned}
A / r_{0}^{2}= & \int_{0 \leqslant \zeta \leqslant 2 \pi / x}\left[1+\left(\partial_{\zeta} \eta\right)^{2}+\left(\frac{\partial_{\varphi} \eta}{1+\eta}\right)^{2}\right]^{1 / 2} \\
& \times(1+\eta) d \varphi d \zeta \\
= & \frac{(2 \pi)^{2}}{x}\left\{1+\frac{\epsilon^{2}}{8}\left(1+\delta_{n 0}\right)\left(x^{2}+n^{2}-1\right)\right\} .
\end{aligned}
$$

Hence with growing disturbance amplitude $\epsilon$ the surface area decreases only if disturbances are axisymmetric $(n=0)$ and $x<1$. In other words, the wavelength of a perturbation must be greater than $\pi$ times the diameter of the jet for it to be unstable. This famous result is due to Plateau (1849). From this it may seem as if disturbances of very long wavelength, i.e., small $x$, are the most rapidly growing ones, as they reduce the surface area the most. But this leaves out the dynamics of the problem, which we have not considered yet. In fact, surface tension has to overcome both inertia and viscous dissipation. The problem first treated by Rayleigh (1879a) is that of a low-viscosity fluid like water, for which inviscid theory applies.

Following Rayleigh, we shall calculate the optimal or fastest growing mode $x_{R}, 0<x_{R}<1$. If a jet is disturbed randomly by external noise, this will usually be the mode that sets the size of drops, as it will soon dominate all the other excited modes. We shall consider only axisymmetric perturbations here, since all nonaxisymmetric perturbations only create more surface area and are therefore stable. For most of this subsection, we shall be looking at inviscid irrotational flow, so the velocity field has a potential $\phi(r, z, t)$, and Eqs. (10), (12), and (13) for an ideal fluid apply.

The velocity potential is nondimensionalized using the initial jet radius $r_{0}$ and the time scale $t_{0}=\left(\rho r_{0}^{3} / \gamma\right)^{1 / 2}$, which implies a balance of surface tension and inertial forces. Hence

$$
\hat{\phi}(\rho, \zeta, \tau)=\frac{t_{0}}{r_{0}^{2}} \phi(r, z, t),
$$

where

$$
\rho=r / r_{0}, \quad \zeta=z / r_{0}, \quad \text { and } \tau=t / t_{0} .
$$

No confusion of the variable $\rho$ with the density should arise here. The nondimensional equations of motion are thus

$$
\begin{aligned}
\Delta \hat{\phi}= & 0, \\
\partial_{\tau} \eta= & \partial_{\rho} \hat{\phi}-\left.\left(\partial_{\zeta} \hat{\phi}\right)\left(\partial_{\zeta} \eta\right)\right|_{\rho=1+\eta} \\
\partial_{\tau} \hat{\phi}= & 1-\frac{1}{2}\left[\left(\partial_{\rho} \hat{\phi}\right)^{2}+\left(\partial_{\zeta} \hat{\phi}\right)^{2}\right] \\
& -\left.\frac{1}{\sqrt{1+\left(\partial_{\zeta} \eta\right)^{2}}}\left[\frac{1}{1+\eta}-\frac{\partial_{\zeta}^{2} \eta}{1+\left(\partial_{\zeta} \eta\right)^{2}}\right]\right|_{\rho=1+\eta} .
\end{aligned}
$$

The initial conditions are

$$
\begin{aligned}
& \eta(\zeta, 0)=\epsilon \cos (x \zeta)-\epsilon^{2} / 4+O\left(\epsilon^{4}\right), \\
& \partial_{\tau} \eta(\zeta, 0)=0 .
\end{aligned}
$$

Since the initial surface displacement is assumed to be proportional to some small parameter $\epsilon$, the velocity will also be small, so we try the expansion

$$
\begin{aligned}
& \hat{\phi}(\rho, \zeta, \tau)=\epsilon \phi_{1}(\rho, \zeta, \tau)+O\left(\epsilon^{2}\right), \\
& \eta(\rho, \zeta, \tau)=\epsilon \eta_{1}(\rho, \zeta, \tau)+O\left(\epsilon^{2}\right) .
\end{aligned}
$$

To first order in $\epsilon$, the equations of motion (27) become

$$
\begin{aligned}
& \left(\partial_{\rho}^{2}+\frac{\partial_{\rho}}{\rho}+\partial_{\zeta}^{2}\right) \phi_{1}=0, \\
& \partial_{\tau} \eta_{1}=\left.\partial_{\rho} \phi_{1}\right|_{\rho=1}, \\
& \partial_{\tau} \phi_{1}=\eta_{1}+\left.\partial_{\zeta}^{2} \eta_{1}\right|_{\rho=1} .
\end{aligned}
$$

Looking for solutions of the form

$$
\begin{aligned}
& \eta_{1}=A_{1}(\tau) \cos (x \zeta), \\
& \phi_{1}=B_{1}(\tau) f(\rho) \cos (x \zeta),
\end{aligned}
$$

we find from Eq. (30a)

$$
f^{\prime \prime}+f^{\prime} / \rho-x^{2} f=0 \text {. }
$$

The only solution that is regular for $\rho=0$ is $f(\rho)=I_{0}(x \rho)$, where $I_{0}$ is a modified Bessel function of the first kind. Substituting this into the boundary conditions (30b) and (30c) gives

$$
\partial_{\tau} A_{1}=B_{1} x I_{1}(x) \text { and } \partial_{\tau} B_{1} I_{0}(x)=A_{1}\left(1-x^{2}\right) .
$$

The solution of this set of equations with the initial conditions (28), namely, $A_{1}(0)=1, \partial_{\tau} A_{1}(0)=0$, is

$$
A_{1}(\tau)=\cosh (\bar{\omega} \tau), \quad B_{1}(\tau)=\frac{\bar{\omega}}{x I_{1}(x)} \sinh (\bar{\omega})
$$

with

$$
\bar{\omega}^{2}=\frac{x I_{1}(x)}{I_{0}(x)}\left(1-x^{2}\right) .
$$

Frequencies are measured in units of the inverse time scale $\omega_{0}=\left(\gamma /\left(\rho r_{0}^{3}\right)\right)^{1 / 2}$. The dimensionless growth rate $\bar{\omega}=\omega / \omega_{0}$ is real for $x<1$, so perturbations grow exponentially, making the jet unstable against arbitrarily small perturbations. For $x>1$, on the other hand, the interface performs oscillations, which will eventually be damped by viscosity. The inviscid dispersion relation (33) is plotted in Fig. 13. The most unstable mode, corresponding to the largest $\bar{\omega}$, occurs at $x_{R}=0.697$. This is the famous Rayleigh mode, which has a wavelength $\lambda_{R}=9.01 r_{0}$.

This result can be checked directly against the observations by Savart (1833). If a jet is excited at the nozzle of a reservoir, it will break with the same frequency with which it is excited. If the impact of the resulting drops on another container is fed back into the reservoir through a mechanical coupling, the original perturbation will be amplified. This amplification is greatest for the most unstable wave numbers, and strong enough for a musical note to sound. Savart measured the pitch of the sound, and from this observation Plateau (1849) inferred a wavelength of 4.38 times the diameter of the jet. Using his concept of maximum instability, Rayleigh (1879b) 


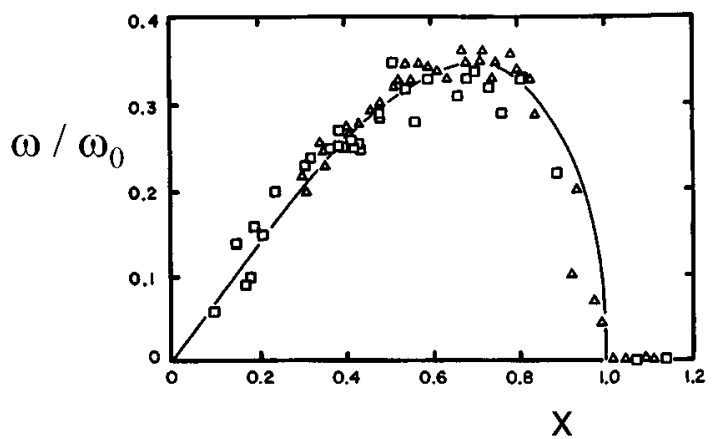

FIG. 13. The dimensionless growth rate of sinusoidal perturbations on a cylinder as a function of the dimensionless wave number. The solid line represents Rayleigh's theory for inviscid flow, the squares are data from Donnelly and Glaberson (1966), triangles are from Goedde and Yuen (1970).

was finally able to interpret this result and found it to be remarkably close to the prediction of his theory.

The first accurate measurement of the complete dispersion relation was done by Donelly and Glaberson (1966). They excited a water jet with a loudspeaker and observed photographically how perturbations grow from one wave crest to the next. Knowing the jet velocity, they could obtain the temporal growth rate of the perturbations. Instead of measuring the height of the crests with respect to $r_{0}$, it is more convenient to take the difference between nodes and crests, which Donelly and Glaberson found to exhibit exponential growth up to one wavelength from the breakup point. This may be due in part to a cancellation of nonlinear effects, as claimed by Yuen (1968) and confirmed by Goedde and Yuen (1970). That is, when one takes the difference between crests and valleys, the contribution from secondorder perturbation theory drops out. Both Donelly and Glaberson (1966) and Goedde and Yuen (1970) found excellent agreement with Rayleigh's dispersion relation to within experimental scatter. The experimental points have been included in Fig. 13. It should be mentioned that an earlier investigation (Crane, Birch, and McCormack, 1964) had found deviations from linear theory based on both the breakup length and direct analysis of photographs. The authors attributed this to the large disturbance amplitude used in their experiment, making linear theory inapplicable.

It was first pointed out by Plateau (1873) that viscosity might considerably alter the above results. As one comes closer and closer to a quasistatic Stokes description, inertia becomes less and less important, and the most unstable wavelength becomes longer, corresponding to the greatest reduction in surface area. Rayleigh himself analyzed the case of very large viscosities (Rayleigh, 1892), assuming that the fluid is described by the Stokes equation. The full problem, using the NavierStokes equation, was first treated in the monograph by Chandrasekhar (1961). The result for $\bar{\omega}$ is a complicated implicit equation, which offers little insight in itself, so it

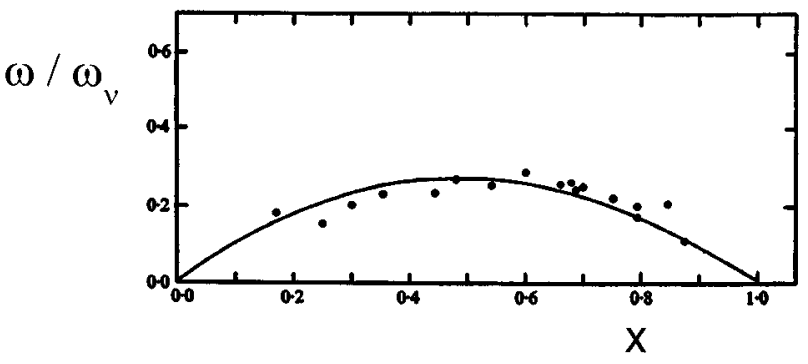

FIG. 14. Growth rates for the decay of a viscous cylinder, comparing data from Goedde and Yuen (1970) with Chandrasekhar's theory. The Reynolds number is $\mathrm{Re}=1.73$.

is not reproduced here. Instead, we give the result that comes from expanding Chandrasekhar's formula for small $x=k r_{0}$. It is

$$
\omega=\omega_{0}\left\{\left[\frac{1}{2} x^{2}\left(1-x^{2}\right)+\frac{9}{4} \mathrm{Re}^{-2} x^{4}\right]^{1 / 2}-\frac{3}{2} \mathrm{Re}^{-1} x^{2}\right\} .
$$

This equation was first obtained by Weber (1931), who analyzed the motion of thin slices of fluid. Surprisingly, it turns out to be a uniformly good approximation for all wavelengths and viscosities. For zero viscosity, it is off by $7 \%$ at most, and becomes increasingly accurate for higher viscosities. According to Eq. (34), the fastestgrowing mode is

$$
x_{R}^{2}=\frac{1}{2+\sqrt{18} / \mathrm{Re}},
$$

which gets smaller with growing viscosity.

As the Reynolds number decreases, the growth rate is eventually determined by a balance of surface tension and viscous forces alone, rather than inertial forces. The dispersion relation (34) gives, in the limit of small Reynolds number,

$$
\omega=\omega_{\nu} \frac{1}{6}\left(1-x^{2}\right),
$$

where $\omega_{\nu}=\gamma /\left(r_{0} \rho \nu\right)$ is a viscous growth rate. The viscous dispersion relation has been tested by Donelly and Glaberson (1966) and Goedde and Yuen (1970), who both found good agreement with Chandrasekhar's (1961) results. A typical dispersion relation is shown in Fig. 14 for $\mathrm{Re}=1.73$.

Since Rayleigh's pioneering work, linear stability of liquid cylinders has been applied to many other physical situations. Tomotika (1935) generalized Rayleigh's (1892) analysis of a highly viscous column to include an outer fluid of finite viscosity and density. Both fluids are described by Stokes' equation. It was found that the most unstable wavelength varied with the ratio of the two viscosities. A simple formula describing the case of two equal viscosities has been given by Stone and Brenner (1996). We shall come back to the problem of breakup of one fluid in another in Sec. VIII.A.

The influence of a surrounding gas of low density was studied by Weber (1931), Lin and Kang (1987), and 
Yang (1992). This is of interest for understanding atomization of liquid jets. The influence of surface charges is included in the work of Schneider et al. (1967) and of Grossmann and Müller (1984). The latter authors also looked at the case of a compressible liquid (Müller and Grossmann, 1985). In an extending liquid column, the axial velocity field is not uniform, but the basic flow has linearly increasing velocity. This case was treated for an inviscid fluid by Frankel and Weihs (1985). Finally, Berger (1988) noted that a general disturbance on a jet has a continuous spectrum of Fourier modes, rather then just a superposition of discrete modes. This alters the evolution of a perturbation for early times, making it deviate from a purely exponential time dependence.

\section{B. Spatial instability}

As we have seen, the temporal instability analyzed in the previous subsection is different from the spatial instability occurring in real jet experiments. So far the perturbation had been assumed to be uniform in space while its temporal growth was observed. In a jet experiment, there is a pointwise disturbance at the nozzle, which grows downstream as it is convected by the mean flow. Thus at a given point in space the size of the perturbation will remain finite, if the convection is fast enough to sweep disturbances away before they can grow. If, however, one considers the frame of reference in which the jet is stationary, it is convected with the disturbances and one observes unlimited growth. This is called "convective instability," as opposed to the case of "absolute instability," where perturbations even grow at a fixed point in space (Landau and Lifshitz, 1984a). Absolute instability implies that no stationary state exists within the realm of linear theory, as any perturbation eventually will grow so much as to make the linear approximation inapplicable.

There are two different physical effects at work which differentiate the spatial from the temporal instability. First, the spatial structure far away from the nozzle is different, as in the former case swells grow from one wave crest to the next. Thus perturbations are described by a superposition of plane waves,

$$
\eta(z, t)=\exp i(k z-\omega t),
$$

where the wave number $k$ is now allowed to be imaginary, while in Rayleigh's theory it was prescribed to be real. The second difference is that the spatial theory is formulated in the half-space $z>0$, with proper boundary conditions at the nozzle opening $z=0$. Usually the jet radius is prescribed to be constant, while the velocity is $v_{j}$ with a small sinusoidal perturbation superimposed on it.

The problem was first studied in a doubly infinite domain by Keller, Rubinow, and Tu (1973). It can be solved by transforming the problem into a coordinate system that moves with the fluid. The plane-wave ansatz in a coordinate system tied to the nozzle is

$$
\eta(\zeta, \tau)=A e^{i(x \zeta-\bar{\omega} \tau)}
$$

$$
\phi(\rho, \zeta, \tau)=B e^{i(x \zeta-\bar{\omega} \tau)} I_{0}(x \rho)+\beta \zeta,
$$

where $\beta$, defined by Eq. (6), is the dimensionless jet velocity. The transformation $\zeta=\zeta^{*}+\beta \tau$ moves the coordinate system such that the jet is at rest, which is the physical situation of the previous subsection. Hence if we write

$$
e^{i(x \zeta-\bar{\omega} \tau)}=e^{i\left(x \zeta^{*}-\omega^{*} \tau\right)},
$$

$\omega^{*}=\bar{\omega}-\beta x$ has to replace $\bar{\omega}$ in the dispersion relation (33).

This means that the new dispersion relation, transformed back to the stationary coordinate system, is

$$
\beta^{2}(\Omega-x)^{2} I_{0}(x)=x I_{1}(x)\left(x^{2}-1\right),
$$

where $\Omega=\bar{\omega} / \beta$ is the experimentally prescribed perturbation frequency at the nozzle, multiplied by $2 \pi t_{0}$. Hence Eq. (38) has to be solved for the complex wave number $x$, which represents the resulting spatial structure.

To relate to the temporal analysis, we analyze Eq. (38) for large $\beta$. Thus we put $x=\Omega+\beta^{-1} x_{1}+O\left(\beta^{-2}\right)$, where the first term describes convection with velocity $\beta$. Comparing powers of $\beta$ one finds

$$
x=\Omega \pm \frac{1}{\beta}\left[\Omega I_{1}(\Omega)\left(\Omega^{2}-1\right) / I_{0}(\Omega)\right]^{1 / 2}+O\left(\beta^{-2}\right) .
$$

Hence up to terms of higher order in $\beta^{-1}$ we recover an expression analogous to Rayleigh's dispersion relation (33), which was to be expected on the grounds of our earlier arguments. For $\Omega<1$, the second term becomes complex, corresponding to an exponentially growing spatial instability. In typical experiments, $\beta$ is larger than 10 , so that terms of higher order in $\beta$ are negligible and Rayleigh's formula can safely be applied. For lower jet velocities corrections eventually become important, but then gravity also becomes relevant and has to be included in the description.

Apart from the roots of Eq. (39) the dispersion relation (38) has an infinite sequence of other roots, already noted by Keller, Rubinow, and Tu (1973), which do not correspond to Rayleigh's solution. To understand their significance, one has to consider the full problem in halfspace, with boundary conditions at the nozzle. This was attempted in a series of papers (Pimbley, 1976; Bogy 1978a, 1978b, 1979b), which correctly analyzed the problem for large Weber numbers. However, the authors failed to notice the absolute instability later found by Leib and Goldstein (1986) for small $\beta$.

Apart from the precise form of the radial velocity profile, which is irrelevant to the qualitative features of the problem, there are two boundary conditions to be satisfied at the nozzle. These are the constant jet diameter and a harmonic time dependence of the velocity field, which excites the jet. Both can be satisfied by superimposing just two traveling waves (37), corresponding to two solutions of Eq. (38). At the other end of the jet, no boundary conditions are available, because the linear 
approximation will eventually break down as disturbances grow. They have to be replaced by physical conditions on the propagation properties of outgoing solutions. One condition is that for a particular branch of solutions the wave number must go to zero as $\Omega \rightarrow 0$, which is obviously the case for the solutions of Eq. (39). This requirement simply means that in a steady state a time-independent boundary condition cannot produce a spatial structure. Expansion of Eq. (38) for small $\Omega$ shows that precisely two such solutions exist for $\Omega \rightarrow 0$, so one ends up with the correct number of boundary conditions at the nozzle.

However, following the two physical branches to large $\Omega$, one finds that they correspond to disturbances with diverging speeds of propagation $\partial \Omega / \partial \operatorname{Re}(\bar{x})$ as $\Omega \rightarrow \infty$. This would mean that energy is transported at arbitrarily high speeds, which is also excluded on physical grounds. Only for $\beta \geqslant \beta_{c}=1.77$ does each solution intersect with another branch of solutions of Eq. (38), which has the correct physical behavior for $\Omega \rightarrow \infty$. Thus steady-state solutions of the linear problem exist for $\beta \geqslant \beta_{c}$. On the other hand, no steady state solution exists for $\beta<\beta_{c}$, the region of absolute instability found by Leib and Goldstein (1986).

Bogy (1978a) advanced similar arguments, but used the existence of a positive group velocity as a criterion to select physical branches. However, Leib and Goldstein (1986) have shown that negative group velocities in fact do exist even in the stationary state. These authors solved the linear problem directly by studying the nozzle problem in much greater detail. In particular, they looked at the influence of different velocity profiles at the nozzle lip, which typically is a Hagen-Poiseuille profile, as opposed to the plug profile analyzed above. For the Hagen-Poiseuille profile the critical jet velocity is even lower, so the issue of absolute instability in jet decay will probably remain an interesting, albeit somewhat academic problem, as gravity will always interfere.

\section{Higher-order perturbative analysis}

Starting from the result of linear theory, the simplest way to explore nonlinear effects is to extend Rayleigh's analysis to higher order in the small-amplitude $\epsilon$. The first such analysis was due to Bohr (1909), who studied the stable oscillations a jet performs around its equilibrium shape if it is released from an orifice with a noncircular cross section. In the following, we are going to concentrate on the axisymmetric case, which is much more difficult, for both stable and unstable ranges of wave numbers exist. As a result, the expansions cannot be expected to be uniformly valid for all wave numbers.

For short-wavelength perturbations, against which the interface is stable, perturbation theory is the obvious way to include nonlinear effects, as the perturbations remain bounded. Hence one can expect to obtain solutions that are uniformly valid in time. For unstable perturbations, however, the perturbation solutions are valid only for short times, because higher-order terms have increasingly fast growth rates. This is not very surprising, as the time scale $t_{0}=\left(\rho r_{0}^{3} / \gamma\right)^{1 / 2}$, on which the linear solution is evolving is of the same order as the breakup time. But obviously the dynamics close to the equilibrium solution do not know about the behavior near breakup, so the solution breaks down when the perturbation is of the same order as the radius itself. So for a description of drop formation other methods are needed. However, there is some hope that as long as the perturbations are small, the nonlinear analysis might describe the growth of higher-order harmonics, which are automatically excited through the nonlinear interaction. If the perturbation expansion is carried out to third order, it will give some indication of the nonuniform breakup of a liquid jet, which produces smaller satellite drops in between the main drops.

The first such theory was attempted by Yuen (1968). The primary prediction of his work is that the boundary between stable and unstable wave numbers is shifted to larger values, as compared to Plateau's result $k r_{0}=1$, if the perturbations have finite amplitude. Thus perturbations that are linearly stable may be unstable because of nonlinear effects. To find this stability boundary, it is sufficient to treat the case of stable oscillations. In the region of unstable wave numbers, the perturbation expansions are far from rigorous. In particular, the coefficients have singularities at "resonant" wave numbers of $k r_{0}=1 / 2$ and possibly others, which are not understood. For that reason, we shall focus on the issue of the nonlinear stability boundary, which is a highly nontrivial problem in itself and has been the subject of some controversy.

Nayfeh (1970) showed that Yuen's treatment of the stability boundary was incorrect, predicting an even lower cutoff wave number. Subsequently, Lafrance (1975) published a theory that predicted no shift in the cutoff at all. This apparent discrepancy was later traced back to an error in his algebra (Chaudhary and Redekopp, 1980). Since the origin of these discrepancies have never been satisfactorily cleared up, we shall give an exposition of the essential features of the theory. Below we show that Nayfeh's (1970) result $x_{c}=1+3 \epsilon^{2} / 4$ for the stability boundary is recovered by modifying Yuen's (1968) original analysis. In the next section this result will also be tested by computer simulation on a onedimensional simplified model (Eggers, 1995b).

To proceed, one writes the velocity potential and the surface deformation as a power series in $\epsilon$ :

$$
\begin{gathered}
\hat{\phi}(\rho, \zeta, \tau)=\sum_{i=1}^{\infty} \epsilon^{i} \phi_{i}(\rho, \zeta, \tau), \\
\eta(\zeta, \tau)=\sum_{i=1}^{\infty} \epsilon^{i} \eta_{i}(\zeta, \tau) .
\end{gathered}
$$

Plugging this into the equations of motion (27) and comparing powers in $\epsilon$, one generates a closed system of equations at each order. The boundary terms are evaluated by expanding $\hat{\phi}$ and $\eta$ around $\rho=1$. Thus the equations at $n$th order have the structure 


$$
\begin{aligned}
& \left(\partial_{\rho}^{2}+\frac{\partial_{\rho}}{\rho}+\partial_{\zeta}^{2}\right) \phi_{n}=0, \\
& \partial_{\tau} \eta_{n}=\partial_{\rho} \phi_{n}+\left.F\left\{\phi_{1}, \eta_{1}, \ldots, \phi_{n-1}, \eta_{n-1}\right\}\right|_{\rho=1}, \\
& \partial_{\tau} \phi_{n}=\eta_{n}+\partial_{\zeta}^{2} \eta_{n}+\left.G\left\{\phi_{1}, \eta_{1}, \ldots, \phi_{n-1}, \eta_{n-1}\right\}\right|_{\rho=1},
\end{aligned}
$$

where $F$ and $G$ are complicated, but calculable, nonlinear functions of the lower-order terms. This allows one to calculate $\phi_{i}$ and $\eta_{i}$ recursively to any order.

Owing to the nonlinear interactions between modes, increasingly higher harmonics of $\cos (x \zeta)$ will be generated, up to $\cos (n x \zeta)$ at $n$th order. Following Yuen (1968), we shall pursue this procedure to third order, which is where the first corrections to the linear stability boundary come in. Corrections of typographical errors in Yuen's original work are given in Rutland and Jameson (1971) and Taub (1976).

The structure of the second- and third-order terms is

$$
\begin{aligned}
& \eta_{2}(\eta, \tau)=A_{22}(\tau) \cos (2 x \zeta)+A_{20}(\tau), \\
& \phi_{2}(\rho, \eta, \tau)=B_{22}(\tau) I_{0}(2 x \rho) \cos (2 x \zeta)+B_{20}(\tau),
\end{aligned}
$$

and

$$
\begin{aligned}
& \eta_{3}(\eta, \tau)=A_{33}(\tau) \cos (3 x \zeta)+A_{31}(\tau) \cos (x \zeta), \\
& \phi_{3}(\rho, \eta, \tau)= B_{33}(\tau) I_{0}(3 x \rho) \cos (3 x \zeta) \\
&+B_{31}(\tau) I_{0}(x \zeta) \cos (x \zeta),
\end{aligned}
$$

where the coefficients $A_{i j}$ and $B_{i j}$ are complicated functions of time and the reduced wave number $x$ (Yuen, 1968; Rutland and Jameson, 1971; Taub, 1976). At $n$th order, they contain terms $\cosh (n \bar{\omega} \tau)$ and $\sinh (n \bar{\omega} \tau)$, so the corresponding perturbation is proportional to $\epsilon^{n} \exp (n \bar{\omega} \tau)$. Thus for unstable wave numbers $x<1$ the expansion is valid only for times $\tau \ll-\log (\epsilon / \bar{\omega})$. If the leading-order perturbation is of order one, which is the case near breakup, all other contributions will be of order one as well. Note also that the coefficients contain singularities at subharmonic values of the cutoff wave number. For example, $A_{22}(\tau)$ has a pole at $x=1 / 2$.

But even for $x>1$ the expansion is not uniformly valid in time, even though for $\epsilon \rightarrow 0$ the solution should be stable and all coefficients should be bounded. In fact, $A_{31}(\tau)$ contains so-called secular terms, which grow linearly in time and thus invalidate the solution for $t \gtrsim \epsilon$. The reason is that the third-order solution is convected and stretched by the first-order contribution. This condition cannot be represented by harmonics of finite order, but rather one needs contributions from all orders of the perturbation expansion.

One way around this problem is to reparametrize time and space coordinates according to

$$
\begin{aligned}
& \bar{\tau}=\nu \tau, \\
& \bar{\zeta}=k_{c} \zeta,
\end{aligned}
$$

where $\nu=1+\epsilon^{2} \nu_{2}+\cdots$ and $k_{c}=1+\epsilon^{2} k_{2}+\cdots$ both have expansions in $\epsilon$ itself. This procedure, called the method of strained coordinates (van Dyke, 1975), was first used by Yuen (1968) in the context of the present problem. Organizing the perturbation expansion in the new variables $\bar{\tau}$ and $\bar{\zeta}$ means one has to replace the differential operators $\partial_{\tau}$ and $\partial_{\zeta}$ by $\nu \partial_{\tau}^{-}=\left(1+\epsilon^{2} \nu_{2}+\cdots\right) \partial_{\tau}^{-}$ and $k_{c} \partial_{\bar{\zeta}}=\left(1+\epsilon^{2} k_{2}+\cdots\right) \partial_{\bar{\zeta}}$. By adjusting the free constants $\nu_{2}$ and $k_{2}$, one hopes to remove secular terms at third order. Effectively, one is resumming certain contributions from all orders. It is, however, far from trivial that this resummation procedure works at all orders. We have to be content with the third-order results, but shall check them against numerical simulations later.

If $k_{2}$ is nonzero, this implies a shift in the cutoff wave number, as $\cos (x \zeta)=\cos (\bar{x} \bar{\zeta})$, with $\bar{x}=x / k_{c}$. Thus the growth rate is now

$$
\bar{\omega}_{1}=\left[\frac{\bar{x} I_{1}(\bar{x})}{I_{0}(\bar{x})}\left(1-\bar{x}^{2}\right)\right]^{1 / 2},
$$

which is real for $\bar{x}<1$ or

$$
x<1+\epsilon^{2} k_{2} \text {. }
$$

In the strained coordinates the equation for $A_{31}$, which contains the secular term, is

$$
\begin{aligned}
\partial_{\bar{\tau}}^{2} A_{31}-\bar{\omega}_{1}^{2} A_{31} & =P_{1}(\bar{x}) \cosh \left(\left(\bar{\omega}_{1}+\bar{\omega}_{2}\right) \bar{\tau}\right) \\
& +P_{2}(\bar{x}) \cosh \left(\left(\bar{\omega}_{1}-\bar{\omega}_{2}\right) \bar{\tau}\right) \\
& +P_{3}(\bar{x}) \cosh \left(3 \bar{\omega}_{1} \bar{\tau}\right) \\
& +\left[P_{4}(\bar{x})+\bar{\omega}_{1}^{2} P_{5}(\bar{x}) \nu_{2}\right. \\
& \left.+\left(\bar{\omega}_{1}^{2} P_{6}(\bar{x})-\bar{x}^{4}\right) k_{2}\right] \cosh \left(\bar{\omega}_{1} \bar{x}\right),
\end{aligned}
$$

where

$$
\bar{\omega}_{2}^{2}=2 \frac{I_{1}(2 \bar{x})}{I_{0}(2 \bar{x})} \bar{x}\left(1-4 \bar{x}^{-2}\right) .
$$

The particular solution coming from the last contribution contains the secular term

$$
\begin{aligned}
A_{31}= & \frac{\bar{\tau}}{2 \bar{\omega}_{1}}\left[P_{4}(\bar{x})+\bar{\omega}_{1}^{2} P_{5}(\bar{x}) \nu_{2}\right. \\
& \left.+\left(\bar{\omega}_{1}^{2} P_{6}(\bar{x})-\bar{x}^{4}\right) k_{2}\right] \sinh \left(\bar{\omega}_{1} \bar{x}\right),
\end{aligned}
$$

which grows with $\bar{\tau}$ unless the terms in the angular brackets cancel. Yuen used precisely this condition to determine $\nu_{2}$ and $k_{2}$, setting

$$
\nu_{2}=-\frac{1}{\bar{\omega}_{1}^{2} P_{5}(\bar{x})}\left\{P_{4}(\bar{x})+\left(\bar{\omega}_{1}^{2} P_{6}(\bar{x})-\bar{x}\right) k_{2}\right\} .
$$

Near the cutoff wave number $\bar{x}=1$, this expression has a singularity, since $\bar{\omega}_{1} \rightarrow 0$. To cancel this singularity one puts

$$
k_{2}=P_{4}(1)=\frac{9}{16}
$$

which together with Eq. (45) is Yuen's result for the cutoff wave number.

Yuen overlooked the fact that the term $P_{3}(\bar{x}) \cosh \left(3 \bar{\omega}_{1} \tau\right)$ in Eq. (46) produces additional secular terms as $\bar{\omega}_{1} \rightarrow 0$. That is, since $P_{3}(1)=3 / 16$, Eq. (46) becomes at $\bar{x}=1$ 


$$
\partial_{\bar{\tau}}^{2} A_{31}=\left(P_{1}(1)+P_{2}(1)\right) \cosh \left(\bar{\omega}_{2} \bar{\tau}\right)+\frac{3}{4}-k_{2} .
$$

This means $A_{31}=\left(3 / 4-k_{2}\right) \bar{\tau}^{2}$ up to oscillatory terms. Thus to cancel the secular terms in the cutoff region one has to put $k_{2}=3 / 4$, and the correct cutoff wave number becomes

$$
x_{c}=1+\frac{3}{4} \epsilon^{2} .
$$

This result was first found by Nayfeh (1970), using the method of multiple time scales (Bender and Orszag, 1978).

It should be mentioned that the shift predicted by Eq. (48) is extremely small, so that an experimental verification has not yet been possible. If the perturbation amplitude is $10 \%$ of the radius, the wave number must be known to within one percent. Even simulations have not led to a significant confirmation (Ashgriz and Mashayek, 1995). Therefore we shall test Eq. (48) in the next section within the framework of one-dimensional models, for which the perturbation theory is virtually identical, except for some differences in the coefficients. The cutoff wave number comes out to be the same.

Away from the cutoff wave number in the unstable region, the growth of higher-order harmonics has been measured by Taub (1976), using a highly sensitive surface probe. Treating the Weber number as a free parameter, he found good agreement with Yuen's theory. Ashgriz and Mashayek (1995) also investigated stable oscillations for $x>x_{c}$, plotting the time dependence of various harmonics of the fundamental perturbation. No quantitative comparison with nonlinear theories was attempted. This welcome test of their validity remains a worthwhile objective. Chaudhary and Redekopp's (1980) work differed in two ways from previous investigations: first, they considered an initial perturbation of the velocity field instead of one of the surface. This was to mimic the true excitations of a jet at the nozzle, which couples to the velocity field. Their treatment of the cutoff region was the same as Yuen's, which means they also did not properly take into account the fact that $\bar{\omega}_{1}$ goes to zero as the cutoff is approached. Therefore their treatment of the cutoff is subject to the same criticism, and it would be a worthwhile problem to redo their analysis. The second aspect of their work was to consider the influence of higher-order harmonics in the driving, which to some extent had also been done by Cline and Anthony (1978). Owing to nonlinearities the two inputs interact at higher orders and change the evolution of the jet. We shall discuss this in greater detail in Sec. VII.C on satellite formation.

Finally, we mention the work of Wang (1968), who studied the effect of finite amplitudes on the most unstable wave number. He obtained a correction to Rayleigh's result $x_{R}=0.697$ which depends on the amplitude.

\section{ONE-DIMENSIONAL APPROXIMATIONS}

It seems obvious that the three-dimensional equations describing the nonlinear motion of free-surface flow are hopelessly complicated if analytical solutions are to be obtained. Perturbation theory cannot be extended to the regime close to breakup. One is therefore interested in generating simplified equations that still capture the essential nonlinear physics of the problem. This is possible in situations where the fluid thread is long and thin, so that the fluid flow is directed mostly along the axis and the velocity field is effectively one-dimensional. In particular, this so-called slenderness assumption turns out to be generically valid close to breakup and becomes exact asymptotically close to pinchoff (Eggers, 1993).

Our strategy is to expand the velocity field in the radial direction, whose lowest-order terms should be sufficient to describe the radial motion. Equations of motion are then derived for the expansion coefficients, which depend only on the axial variable $z$. The idea behind this goes back to Reynolds (1886) and is often called the "lubrication approximation." This is because it is traditionally applied to flows in a narrow channel, for instance between two moving mechanical parts. In the direction perpendicular to the surface the velocity field will be parabolic, leaving an equation for the amplitude as a function of the position in the channel.

The situation of free-surface flow, however, is much more complicated. First, the smallness of the expansion parameter will depend on the solution itself, essentially on whether the typical surface height is really small compared to a typical longitudinal extension. Second, it is not obvious which forces in the equations have to be taken into account. In the example of lubrication flow, viscous forces will be balanced by pressure forces, while inertia is not important. But in our case the relative importance of inertial, viscous, or surface-tension forces will depend on the problem considered. Nevertheless, the lubrication approximation for radially symmetric free-surface flow by now has some history of its own, inspired partly by the theory of shallow-water waves, summarized by Peregrine (1972). A broad perspective on approximations for axisymmetric flows is given in Middleman's (1995) book. The beautiful discussion of flows includes jets, waves, drop dynamics, and thin-film flow. Unfortunately, the lubrication equations for a thin jet, to be derived below, are not given correctly.

Matovich and Pearson (1969), as well as Pearson and Matovich (1969) and Schultz and Davis (1982) derived equations for the steady motion of viscous threads in order to study fiber spinning. In a pioneering work, Lee (1974) studied the dynamics of an inviscid liquid jet, including a fully nonlinear numerical computation. This allowed him to look at the nonuniformity of breakup and to estimate the size of satellite drops. Lee's equations were further investigated (Pimbley, 1976; Pimbley and Lee, 1977) to study satellite behavior. The fundamental problem with Lee's equations is, however, that viscosity will become important in the process of pinching, regardless of how small. We shall investigate the spurious singularities of the inviscid equations later in much greater detail (Brenner et al., 1997).

This problem was fixed by Green (1976), who derived equations that included viscosity. His approach was dif- 
ferent from Lee's, as he did not start from the equations of motion, but rather from an inherently onedimensional description, endowed with the correct symmetry properties (Green and Laws, 1966). We shall see below that these so-called Cosserat equations can in fact be obtained from the Navier-Stokes equation by averaging over appropriate slices of the fluid (Dupont, 1993). Again, the Cosserat equations were used in a series of papers (Bogy, 1978a, 1978b, 1979a, 1979b) focusing on linear stability, and in numerical simulations (Bogy, Shine, and Talke, 1980; Shield, Bogy, and Talke, 1986). The same equations were also used extensively in the context of liquid bridges (Meseguer, 1983; Meseguer and Sanz, 1985; Perales and Meseguer, 1992).

But it was not until recently that a systematic derivation of viscous one-dimensional equations was carried out starting from the Navier-Stokes equation. Bechtel, Forest, and Lin (1992) used a perturbative approach to obtain one-dimensional equations to arbitrarily high order, which we shall explain in Sec. V.A. Independently, Eggers and Dupont (1994) and Garcia and Castellanos (1994) gave essentially the same leading-order equations. At higher orders, different approaches are possible. In Sec. V.B we shall explain how the onedimensional Cosserat equations are obtained from the Navier-Stokes equation.

\section{A. Radial expansion method}

We want to find approximate solutions to the equations of motion in a situation where the typical thickness $\ell_{r}$ of the fluid neck is small compared with a typical axial scale $\ell_{z}$. This means the problem contains a small parameter $\epsilon$ which relates the two scales

$$
\ell_{r}=\epsilon \ell_{z} .
$$

In addition to the length scales $\ell_{r}$ and $\ell_{z}$ the problem is characterized by a time scale $\tau$. These scales can now be used to estimate the order of magnitude of the different terms in the equations of motion. We begin by saying that the axial component of the velocity field is approximated by $v_{z} \approx \ell_{z} / \tau$, which may also be viewed as the definition of $\tau$. By continuity, we have $\partial_{z} v_{z} \approx \partial_{r} v_{r}$, and hence $v_{r} \approx \epsilon \ell_{z} / \tau$. The motion is driven by surface tension, so the main contribution to the pressure comes from the mean curvature, $p / \rho \approx(\gamma / \rho) \kappa$. For an almost flat interface, $\kappa$ is dominated by the radius of curvature perpendicular to the axis, and hence $\kappa \approx 1 / \ell_{r}$ and $p / \rho \approx(\gamma / \rho) / \ell_{r}$. From the Navier-Stokes equation we have $\partial_{t} v_{z} \approx \partial_{z} p / \rho$, implying that $\gamma / \rho \approx \ell_{r} \ell_{z}^{2} / \tau^{2}=\epsilon \ell_{z}^{3} / \tau^{2}$. So, if surface tension has this order of magnitude, surface tension and inertial forces are balanced.

We have already mentioned that the inviscid equations develop singularities in finite time, so surface tension and inertial forces must also be balanced by viscosity for a consistent theory. We expect $v_{z}$ to have the greatest variations in the $z$ direction, so the dominant viscous term is $\nu \partial_{z}^{2} v_{z} \approx \nu /\left(\ell_{z} \tau\right)$. Equating $\nu /\left(\ell_{z} \tau\right)$ with $\partial_{t} v_{z}$ we find that $\nu \approx \ell_{z}^{2} / \tau$ is the viscosity at which viscous forces are balanced by inertia.
For a given problem, $\nu$ and $\gamma / \rho$ are of course given numbers, so physically speaking the scales $\ell_{r}, \ell_{z}$, and $\tau$ of a particular solution adjust themselves to make various terms in the equations balance. For example, to avoid an inviscid singularity, $\ell_{z}$ and $\tau$ will come out to ensure $\nu \approx \ell_{z}^{2} / \tau$. From the constancy of the physical parameters $\nu$ and $\gamma / \rho$ we can therefore deduce that $\ell_{z}^{2} \approx \nu \tau$ and $\ell_{r} \approx \gamma \tau /(\rho \nu)$, so we get $\ell_{z} \approx\left(\nu^{2} \rho / \gamma\right) \epsilon$, $\ell_{r} \approx\left(\nu^{2} \rho / \gamma\right) \epsilon^{2}$, and $\tau \approx\left(\nu^{3} \rho^{2} / \gamma^{2}\right) \epsilon^{2}$. If the typical scales of a solution behave in this way, surface-tension, inertial, and viscous forces will be balanced during the entire evolution of this solution.

Having estimated the typical size of all terms, it is useful to choose the scales $\ell_{z}, \ell_{r}$, and $\tau$ such that

$$
\ell_{z}=\epsilon \ell_{\nu}, \quad \ell_{r}=\epsilon^{2} \ell_{\nu}, \quad \text { and } \tau=\epsilon^{2} t_{\nu},
$$

with

$$
\ell_{\nu}=\frac{\nu^{2} \rho}{\gamma}, \quad t_{\nu}=\frac{\nu^{3} \rho^{2}}{\gamma^{2}} .
$$

These are the unique length and time scales that can be formed from the material parameters. When we use $\ell_{z}, \ell_{r}$, and $\tau$ to nondimensionalize all variables according to

$$
\begin{aligned}
& r=\ell_{r} \tilde{r}, \quad z=\ell_{z} \tilde{z}, \quad t=\tau \tilde{t}, \\
& v_{r}=\frac{\ell_{r}}{\tau} \widetilde{v}_{r}, \quad v_{z}=\frac{\ell_{z}}{\tau} \widetilde{v}_{z}, \quad p / \rho=\frac{\ell_{z}^{2}}{\tau^{2}} \tilde{p}, \\
& h=\ell_{r} \tilde{h},
\end{aligned}
$$

all material parameters drop out of the problem and the rescaled equations of motion become

$$
\begin{aligned}
& \partial_{\tilde{t}} \tilde{v}_{r}+\tilde{v}_{r} \partial_{r} \tilde{v}_{r}+\tilde{v}_{z} \partial_{\tilde{z}} \tilde{v}_{r}=-\frac{1}{\epsilon^{2}} \partial_{r} \tilde{p}+\frac{1}{\epsilon^{2}} \partial_{\tilde{r}}^{2} \widetilde{v}_{r}+\partial_{\tilde{z}}^{2} \widetilde{v}_{r} \\
& +\frac{1}{\epsilon^{2}} \partial_{r} \widetilde{v_{r}} / \tilde{r}-\frac{1}{\epsilon^{2}} \widetilde{v_{r}} / \tilde{r}^{2} \\
& \partial_{t} \tilde{v_{z}}+\tilde{v}_{r} \partial_{r} \tilde{v_{z}}+\tilde{v}_{z} \partial_{z} \widetilde{v_{z}}=-\partial_{z} \tilde{p}+\frac{1}{\epsilon^{2}}\left(\partial_{\tilde{r}}^{2} \widetilde{v}_{z}+\partial_{r} \tilde{v_{z}} / \widetilde{r}\right) \\
& +\partial_{z}^{2} \tilde{v_{z}} .
\end{aligned}
$$

The equation of continuity is

$$
\partial_{\tilde{r}} \widetilde{v_{r}}+\partial_{\tilde{z}} \widetilde{v_{z}}+\widetilde{v_{r}} / \tilde{r}=0 \text {, }
$$

and the tangential and normal force balances read

$$
\begin{aligned}
& \tilde{p}-\frac{2}{1+\epsilon^{2}\left(\partial_{z} \tilde{h}\right)^{2}}\left[\partial_{\tilde{r}} \widetilde{v}_{r}+\epsilon^{2} \partial_{\tilde{z}} \widetilde{v}_{z}\left(\partial_{z} \tilde{h}\right)^{2}\right. \\
& \left.-\left(\partial_{\tilde{r}} \tilde{v}_{z}+\epsilon^{2} \partial_{\tilde{z}}-\widetilde{v}_{r}\right) \partial_{\bar{z}} \tilde{h}\right]=\left.\widetilde{\kappa}\right|_{\tilde{r}=\tilde{h}}, \\
& 2 \epsilon^{2}\left(\partial_{r} \widetilde{v_{r}}-\partial_{\tilde{z}} \widetilde{v_{z}}\right) \partial_{\tilde{z}} \tilde{h}+\left(\partial_{\tilde{r}} \widetilde{v_{z}}+\epsilon^{2} \partial_{\tilde{z}} \widetilde{v_{r}}\right)\left(1-\epsilon^{2}\left(\partial_{\bar{z}} \tilde{h}\right)^{2}\right) \\
& =\left.0\right|_{\tilde{r}=\tilde{h}} \text {. }
\end{aligned}
$$

Finally, the kinematic boundary condition is

$$
\partial_{\bar{t}} \tilde{h}+\widetilde{v}_{z} \partial_{\tilde{z}} \tilde{h}=\left.\widetilde{v_{r}}\right|_{\tilde{r}=\tilde{h}} .
$$


We now write the two components of the velocity field and the pressure field as a power series:

$$
\begin{aligned}
& \tilde{v}_{z}(\tilde{r}, \widetilde{z}, \widetilde{t})=\widetilde{v_{0}}(\widetilde{z}, \widetilde{t})+\widetilde{v}_{2}(\widetilde{z}, \tilde{t})(\epsilon \widetilde{r})^{2}+\cdots, \\
& \widetilde{v_{r}}(\tilde{r}, \tilde{z}, \tilde{t})=-{\widetilde{v_{0}}}_{0}^{\prime}(\widetilde{z}, \widetilde{t}) \frac{\epsilon \tilde{r}}{2}-\widetilde{v_{2}^{\prime}}(\tilde{z}, \widetilde{t}) \frac{(\epsilon \widetilde{r})^{3}}{4}-\cdots, \\
& \widetilde{p}(\tilde{r}, \tilde{z}, \tilde{t})=\tilde{p_{0}}(\widetilde{z}, \widetilde{t})+\widetilde{p_{2}}(\widetilde{z}, \tilde{t})(\epsilon \widetilde{r})^{2}+\cdots .
\end{aligned}
$$

In Eq. (57), $\widetilde{v_{r}}$ has already been chosen to make the velocity field incompressible. The prime refers to differentiation with respect to $\widetilde{z}$. Inserting the expansions into the equations of motion (53), we find

$$
\partial_{\tilde{t}} \widetilde{v_{0}}+\widetilde{v_{0}} \widetilde{v_{0}^{\prime}}=-{\widetilde{p_{0}^{\prime}}}^{\prime}+\left(4 \widetilde{v_{2}}+\widetilde{v_{0}^{\prime \prime}}\right)+O\left(\epsilon^{2}\right)
$$

from the $v_{z}$ equation, while the equation for $v_{r}$ is satisfied identically to leading order $\epsilon^{-1}$. The two boundary conditions (55) give

$$
\begin{aligned}
& \widetilde{p_{0}}=\tilde{\kappa}-\widetilde{v_{0}^{\prime}}+O\left(\epsilon^{2}\right) \quad \text { and } \\
& -3 \widetilde{v}_{0}^{\prime} \tilde{h}^{\prime}-\frac{1}{2} \widetilde{v_{0}^{\prime \prime}} \tilde{h}+2 \widetilde{v_{2}} \tilde{h}+O\left(\epsilon^{2}\right)=0,
\end{aligned}
$$

which can be read as an equation for $\widetilde{p_{0}}$ and $\widetilde{v_{2}}$, resulting in

$$
\partial_{t} \widetilde{v_{0}}+\widetilde{v_{0}}{\widetilde{v_{0}^{\prime}}}_{0}^{\prime}=-\tilde{\kappa}+3 \frac{\left(\widetilde{v_{0}^{\prime}} \tilde{h}^{2}\right)^{\prime}}{\widetilde{h^{2}}}
$$

to leading order. The factor of 3 in front of the viscous term is typical for elongational flows, and $3 \nu$ is often called the Trouton viscosity (Trouton, 1906). The equation of motion (56) for $\tilde{h}$ immediately gives

$$
\partial_{\tilde{t}} \tilde{h}+\widetilde{v}_{0} \tilde{h}^{\prime}=-\frac{1}{2} \tilde{h} \widetilde{v}_{0}^{\prime} .
$$

The mean curvature $\tilde{\kappa}$ appearing in Eq. (59) is

$$
\tilde{\kappa}=\frac{1}{\tilde{h}\left(1+\epsilon^{2} \tilde{h}^{\prime 2}\right)^{1 / 2}}-\epsilon^{2} \frac{\tilde{h}^{\prime \prime}}{\left(1+\epsilon^{2} \tilde{h}^{\prime 2}\right)^{3 / 2}},
$$

and thus to leading order

$$
\tilde{\kappa}=\frac{1}{\tilde{h}}+O\left(\epsilon^{2}\right)
$$

The system (59), (60), and (62) constitutes the desired lubrication approximation to leading order. Remarkably, it turns out to be a closed system for the onedimensional velocity field $\widetilde{v_{0}}(\widetilde{z}, \widetilde{t})$ and the height $\tilde{h}(\tilde{z}, \tilde{t})$. Besides reducing the number of dimensions by one, this eliminates the free-surface boundary conditions from the problem. For $\epsilon \rightarrow 0$, it is an exact representation of the full Navier-Stokes dynamics and will be central to our subsequent discussion.

The leading-order equations are part of a perturbation expansion that can be extended to arbitrarily high order in $\epsilon$ (Bechtel et al. 1995; Bechtel, Carlson, and
Forest, 1995). To this end the expansions (57) are inserted into the equation of motion and contributions of the same power in $r$ are collected. For example, the next contribution to the axial momentum equation following (58) is of order $r^{2}$, giving

(57) $\partial_{t} \widetilde{v_{2}}+\widetilde{v_{0}}{\widetilde{v_{2}^{\prime}}}^{\prime}=-\tilde{p_{2}^{\prime}}+\left(16{\widetilde{v_{4}}}_{4}+{\widetilde{v_{2}^{\prime \prime}}}_{2}\right)$.

To obtain a closed system, one uses the boundary equations (55) and (56) and solves to each order in $\epsilon$. Unfortunately, this leads to closure only if $\tilde{h}$ and the Taylor coefficients $\widetilde{v_{i}}$ of the velocity field are expanded as well (Bechtel et al., 1995):

$$
\begin{aligned}
& \widetilde{v_{i}}(\widetilde{z}, \widetilde{t})=\sum_{j=0}^{\infty} \widetilde{v}_{i}^{(j)}(\widetilde{z}, \widetilde{t}) \epsilon^{j}, \\
& \tilde{h}(\tilde{z}, \tilde{t})=\sum_{j=0}^{\infty} \tilde{h}^{(j)}(\tilde{z}, \widetilde{t}) \epsilon^{j} .
\end{aligned}
$$

Comparing powers of $\epsilon$ in each of the boundary equations (55), (56) and in the momentum equations up to order $r^{i}$, one obtains a closed system for the coefficients ${\tilde{v_{i}}}_{i}^{(j)}$ and $\tilde{h}^{(j)}$ at any order in $\epsilon$. This double-expansion strategy thus leads to quadratic growth in the number of equations with order. We refer the reader to Bechtel, Carlson, and Forest (1995) for the explicit equations at higher orders.

Solutions to these systems are obtained in the following manner: First one has to solve the leading-order sys(59)em, Eqs. (59), (60), with appropriate boundary and initial conditions. The solutions of the leading-order equations are then plugged into the equations at the next order, which again can be integrated to compute a correction to the leading-order solution. This process can be continued to obtain solutions up to any given order. The problem with this approach is that one is confined to situations in which the leading-order equa60) tions already give a uniformly valid approximation of the problem, since perturbation theory can only describe small corrections to the leading behavior.

By contrast, Garcia and Castellanos (1994) derive a nonlinear system of equations for the expansion coefficients of the velocity field, which are solved simultaneously. Thus the more accurate description of the radial dependence of the velocity field feeds back into the leading-order amplitudes. While the resulting systems of equations get more and more complicated at higher orders, the method is tremendously powerful computationally. Indeed, one expects the results to be uniformly valid as long as the radial dependence of the expansion is fine enough to describe a given flow structure accurately. For example, at the next-to-leading order the $z$ component of the velocity field is written as

$$
\begin{aligned}
\tilde{v}_{z}(\tilde{r}, \tilde{z}, \widetilde{t}) & =\widetilde{v_{0}}(\widetilde{z}, \tilde{t})+\widetilde{v_{2}}(\widetilde{z}, \widetilde{t})(\epsilon \widetilde{r})^{2} \\
& +\widetilde{v}_{4}(\tilde{z}, \widetilde{t})(\epsilon \widetilde{r})^{4}+\cdots .
\end{aligned}
$$

Equations (58), (63), and the corresponding radial momentum equations form a coupled set of equations, which are solved simultaneously. Boundary-force balance is used to eliminate $\widetilde{v_{4}}$ and $\widetilde{p_{2}}$, resulting in a coupled system of three equations for $\widetilde{v_{0}}, \widetilde{v_{2}}$, and $\widetilde{h_{0}}$. 


\section{B. Averaging method: Cosserat equations}

In this subsection, we describe an entirely different approach to obtaining one-dimensional approximations, based on integrating the equations of motion over a slice of the fluid. Interestingly, the leading-order versions of this procedure lead to a set of equations well known as the Cosserat equations (Green, 1976). In an approach borrowed from solid mechanics (Green and Laws, 1966), one starts from a system of one-dimensional fields, called "directors." The equations of motion are then derived from symmetry considerations and conservation properties and they coincide with the equations derived from the Navier-Stokes equation by integrating over slices. Ultimately, this is not surprising, as the momentum over a slice of the fluid must inherit the correct symmetry properties from the full equations of motion. The obvious advantage of this derivation (Dupont, 1993), presented here for the first time, is that there is a clear connection to the three-dimensional equations of motion. Therefore the error made at a given order of the approximation can be estimated and can be systematically improved by going to a higher order of the approximation. Methods that are very similar in spirit, but lead to a coupled set of integro-differential equations, have been developed by Entov and Yarin starting from the early 1980s (Yarin, 1993) and by Dewynne, Howell, and Wilmott (1994). They are more general in that they allow for an arbitrary position of the centerline of the jet as well as noncircular cross sections.

Dupont's procedure is essentially a Galerkin approximation of the equations of motion, which means the velocity is represented by a superposition of a finite number of basis functions. Equations of motion for the coefficients are obtained by projecting the NavierStokes equation onto each of the basis functions. Again, a coupled system of equations results, which should represent the velocity field more and more accurately at higher orders.

The basis functions used represent slices of the fluid,

$$
\mathbf{w}^{(2 i, \bar{z})}(r, z)=\left(\begin{array}{c}
-\frac{r^{2 i+1}}{2 i+2} \delta^{\prime}(z-\bar{z}) \\
r^{2 i} \delta(z-\bar{z})
\end{array}\right),
$$

which are divergence free, with a discrete index $i$ and a continuous index $z$ representing the position of the slice. The main deficiency of the functions (65) is that they are not orthogonal for different $i$, which is the price one has to pay for their being both perfectly localized in space and divergence free. Using Eq. (65), we can write any incompressible velocity field as a unique superposition of basis functions:

$$
\mathbf{v}(r, z, t)=\sum_{i=0}^{\infty} \int_{-\infty}^{\infty} v^{(2 i)}(\bar{z}, t) \mathbf{w}^{\left(2 i, z^{-}\right)}(r, z) d \bar{z} .
$$

Now one has to plug Eq. (66), truncated at some order $i=n$, into the Navier-Stokes equation and the kinematic boundary condition and project it onto the corre- sponding $n+1$ basis functions $\mathbf{w}^{\left(2 i, z^{-}\right)}(r, z), i=0, \ldots, n$. This yields a coupled set of $(n+2)$ equations for the amplitudes $v^{(0)}(\bar{z}, t), \ldots, v^{(2 n)}(\bar{z}, t)$, and $h(\bar{z}, t)$. To project the Navier-Stokes equation onto an arbitrary incompressible vector field $\mathbf{w}(r, z)$, we write it in the form

$$
\rho\left(\partial_{t} \mathbf{v}+(\mathbf{v} \nabla) \mathbf{v}\right)=\nabla \cdot \boldsymbol{\sigma},
$$

where the stress tensor is given by

$$
\sigma_{i j}=-p \delta_{i j}+2 \eta D_{i j}(\mathbf{v})
$$

and

$$
D_{i j}(\mathbf{v})=\frac{1}{2}\left(\partial_{j} v_{i}+\partial_{i} v_{j}\right)
$$

is the deformation tensor. We multiply the NavierStokes equation by $\mathbf{w}(r, z)$ and integrate over the volume of the fluid, $0 \leqslant r \leqslant h(z)$. The result can be written symbolically as

$$
\rho I_{\rho}=I_{\sigma}
$$

where

$$
\begin{aligned}
& I_{\rho}=\int_{V}\left[\partial_{t} \mathbf{v}+(\mathbf{v} \nabla) \mathbf{v}\right] \mathbf{w} d^{3} x \quad \text { and } \\
& I_{\sigma}=\int_{V}(\nabla \boldsymbol{\sigma}) \mathbf{w} d^{3} x .
\end{aligned}
$$

On integrating by parts, $I_{\sigma}$ can be written as

$$
\begin{aligned}
I_{\sigma}= & \int_{V} \partial_{i} \sigma_{i j} w_{j} d^{3} x=-\int_{V} \sigma_{i j} \partial_{i} w_{j} d^{3} x \\
& +\int_{\partial V} n_{i} \sigma_{i j} w_{j} d^{2} s .
\end{aligned}
$$

In the first integral on the right the pressure does not contribute, since $\partial_{i} w_{i}=0$. For the second integral we use the force balance at the boundary,

$$
n_{i} \sigma_{i j}=-\gamma \kappa n_{j} .
$$

Thus after some manipulations we obtain

$$
I_{\sigma}=-2 \eta I_{\eta}-\gamma I_{\gamma},
$$

where

$$
I_{\eta}=\int_{V} D_{i j}(\mathbf{v}) D_{i j}(\mathbf{w}) d^{3} x, \quad I_{\gamma}=\int_{\partial V} \kappa(\mathbf{n w}) d^{2} s,
$$

and the desired projection of the Navier-Stokes equation is in full

$$
\begin{aligned}
\rho \int_{V}\left[\partial_{t} \mathbf{v}+(\mathbf{v} \nabla) \mathbf{v}\right] \mathbf{w} d^{3} x= & -\gamma \int_{\partial V} \kappa(\mathbf{n w}) d^{2} s \\
& -2 \eta \int_{V} D_{i j}(\mathbf{v}) D_{i j}(\mathbf{w}) d^{3} x .
\end{aligned}
$$

To be more explicit, we compute the leading-order approximation, so 


$$
\mathbf{w}(r, z):=\mathbf{w}^{(0, \bar{z})}(r, z)=\left(\begin{array}{c}
-\frac{r}{2} \delta^{\prime}(z-\bar{z}) \\
\delta(z-\bar{z})
\end{array}\right)
$$

and

$$
\mathbf{v}(r, z, t)=\left(\begin{array}{c}
-\frac{r}{2} v_{0}^{\prime}(z, t) \\
v_{0}(z, t)
\end{array}\right) .
$$

For the integrals we find

$$
\begin{aligned}
\frac{1}{\pi} I_{\rho}= & h^{2} \dot{v}_{0}-\frac{1}{8}\left(h^{4} \dot{v}_{0}^{\prime}\right)^{\prime}+h^{2} v_{0} v_{0}^{\prime}+\frac{1}{16}\left(h^{4} v_{0}^{\prime 2}\right)^{\prime} \\
& -\frac{1}{8}\left(h^{4} v_{0} v_{0}^{\prime \prime}\right)^{\prime}
\end{aligned}
$$

and

$$
\frac{1}{\pi} I_{\eta}=-\frac{3}{2}\left(h^{2} v_{0}^{\prime}\right)^{\prime}+\frac{1}{16}\left(h^{4} v_{0}^{\prime \prime}\right)^{\prime \prime},
$$

where the dot represents a time derivative.

The surface integral $I_{\gamma}$ is most conveniently evaluated using Gauss' theorem. To do this, we formally define $\kappa$ over the total volume: $\kappa(r, z)=\kappa(z)$, hence

$$
\begin{aligned}
I_{\gamma} & =\int_{V} \partial_{i}\left(\kappa w_{i}\right) d^{3} x=\int_{V}\left(\partial_{i} \kappa\right) w_{i} d^{3} x \\
& =\int_{V}\left(\partial_{z} \kappa\right) w_{z} d^{3} x .
\end{aligned}
$$

We end up with the equation

$$
\begin{gathered}
\rho\left[h^{2} \dot{v}_{0}-\frac{1}{8}\left(h^{4} \dot{v}_{0}^{\prime}\right)^{\prime}+h^{2} v_{0} v_{0}^{\prime}+\frac{1}{16}\left(h^{4} v_{0}^{\prime 2}\right)^{\prime}-\frac{1}{8}\left(h^{4} v_{0} v_{0}^{\prime \prime}\right)^{\prime}\right] \\
=-\gamma h^{2} \kappa^{\prime}+\eta\left[3\left(h^{2} v_{0}^{\prime}\right)^{\prime}-\frac{1}{8}\left(h^{4} v_{0}^{\prime \prime}\right)^{\prime \prime}\right]
\end{gathered}
$$

Plugging the velocity field (73) into the kinematic boundary condition, one obtains

$$
\dot{h}+v_{0} h^{\prime}=-\frac{1}{2} v_{0}^{\prime} h,
$$

which is the corresponding equation for $h$. Equations (74) and (75) are precisely the Cosserat equations as given by Bogy (1979b). One immediately notices that for a slender jet, for which $h$ is of order $\epsilon$, one recovers the leading-order equations (59), (60). This is the first a time a connection between the Navier-Stokes equations and the Cosserat equations has been established. Bousfield et al. (1986) miss some of the inertial and the viscous terms. Perales and Meseguer (1992) selectively take some higher-order terms into account, starting from a radial expansion. Schulkes (1993) treats only the inviscid case.

The next step in the expansion is to approximate the velocity field as

$$
\mathbf{v}(r, z, t)=\left(\begin{array}{c}
-\frac{r}{2} v_{0}^{\prime}(z, t)-\frac{r^{3}}{4} v_{2}^{\prime}(z, t) \\
v_{0}(z, t)+r^{2} v_{2}(z, t)
\end{array}\right) .
$$

Evaluating the projection (71) with the two basis functions

$$
\begin{aligned}
& \mathbf{w}^{(0, \bar{z})}(r, z)=\left(\begin{array}{c}
-\frac{r}{2} \delta^{\prime}(z-\bar{z}) \\
\delta(z-\bar{z})
\end{array}\right), \\
& \mathbf{w}^{(2, \bar{z})}(r, z)=\left(\begin{array}{c}
-\frac{r^{3}}{4} \delta^{\prime}(z-\bar{z}) \\
r^{2} \delta(z-\bar{z})
\end{array}\right),
\end{aligned}
$$

one obtains a coupled system for $v_{0}$ and $v_{2}$, while the equation for $h$ is now

$$
\dot{h}+v_{0} h+v_{2} h^{3}=-\frac{h}{2} v_{0}^{\prime}-\frac{h^{3}}{4} v_{2}^{\prime} .
$$

This represents the analogue of the coupled set of equations derived by Garcia and Castellanos (1994).

Note, however, that we did not make any assumption about $h$ 's being small, but used only an expansion of the velocity field. In particular, one gets to keep the full curvature term $\kappa$ coming from the surface forces even at leading order; see Eq. (74). In the radial expansion method, $h$ had to be of order $\epsilon$, so $\kappa$ is approximated by $1 / h$ as in Eq. (62). There are good reasons for keeping the full curvature term even in Eq. (59) (Eggers and Dupont, 1994; Garcia and Castellanos, 1994), but only the Galerkin method offers a consistent rationale for doing so.

\section{Basic properties and simulations}

Since we shall be dealing mostly with the leadingorder system (59), (60), we shall repeat it here in dimensional form in order to make the origin of the different terms more apparent. Thus

$$
\begin{aligned}
& \partial_{t} v+v v^{\prime}=-p^{\prime} / \rho+3 \nu \frac{\left(h^{2} v^{\prime}\right)^{\prime}}{h^{2}}, \\
& \partial_{t} h+v h^{\prime}=-v^{\prime} h / 2,
\end{aligned}
$$

where $v$ is the leading-order expansion coefficient of $v_{z}$. The "pressure" $p(z, t)$ here is just its contribution coming from the mean curvature, whose leading-order contribution is

$$
p=\gamma \frac{1}{h} \text {. }
$$

Although the greatest significance of the system (79)(81) lies in the asymptotic limit close to breakup, it has also been successfully used for simulations away from 
breakup (Eggers and Dupont, 1994; Brenner, Shi, and Nagel, 1994). The key to this success lies in a modification of Eq. (81) to include the complete expression for the mean curvature,

$$
p=\gamma\left(\frac{1}{h\left(1+h^{\prime 2}\right)^{1 / 2}}-\frac{h^{\prime \prime}}{\left(1+h^{\prime 2}\right)^{3 / 2}}\right) .
$$

This procedure was already adopted by Lee (1974), and has subsequently been used by many other authors (Meseguer, 1983; Johnson et al., 1991; Garcia and Castellanos, 1994). The best rationale for doing so is offered by the Galerkin procedure of the previous subsection, but Eq. (79) has the advantage of much greater mathematical simplicity over Eq. (74). The complete curvature on the right-hand side of Eq. (82) is of course also represented as an infinite sequence of terms in the equations of the radial expansion. It would be extremely desirable to develop a general and consistent method to "resum" all the relevant terms of the expansion, such that Eq. (82) is included even at leading order. Perturbative resummation like this is customary in quantum field theory (Amit, 1978), but has not yet been explored in the context of nonlinear partial differential equations.

The system (79),(80) has to be supplemented with boundary conditions

$$
\begin{aligned}
& h\left(z_{ \pm}, t\right)=h_{ \pm}(t), \\
& v\left(z_{ \pm}, t\right)=v_{ \pm}(t),
\end{aligned}
$$

where the two boundary points $z_{+}, z_{-}$may themselves be time dependent. In a liquid-bridge experiment, for example, $z_{ \pm}$represent the position of the two rings holding the bridge.

The two most important conservation laws are conservation of mass and energy,

$$
\partial_{t} V=\left.\pi h^{2} v\right|_{z=z_{-}} ^{z_{+}},
$$

with the volume being

$$
V=\pi \int_{z_{-}}^{z_{+}} h^{2} d z
$$

expressing conservation of mass. The kinetic energy is

$$
E_{\mathrm{kin}}=\frac{\pi}{2} \rho \int_{z_{-}}^{z_{+}} h^{2} v^{2} d z
$$

while the potential energy is $\gamma$ times the total surface area:

$$
E_{\mathrm{pot}}=2 \pi \gamma \int_{z_{-}}^{z_{+}} h\left(1+h^{\prime 2}\right)^{1 / 2} d z
$$

Thus the sum obeys the balance equation

$$
\partial_{t}\left(E_{\text {kin }}+E_{\text {pot }}\right)=D+\text { boundary terms, }
$$

where $D$ is the rate of energy dissipation,

$$
D=-3 \pi \nu \rho \int_{z_{-}}^{z_{+}}\left(h v^{\prime}\right)^{2} d z
$$

This means that apart from driving through boundary terms, the total energy of the system can only decrease, and a drop eventually has to reach some static equilibrium shape, corresponding to a minimum of the potential energy. Since the potential energy (87) is the same as in the full equations, the physical equilibrium states such as those of a drop hanging from a faucet (Michael and Williams, 1976), will also be reproduced exactly. For reproducing the equilibrium states it is of course crucial to keep the full curvature term as in Eq. (82).

Another important benefit of the "resummed" form of the pressure, Eq. (82), emerges when considering the linear stability around a cylindrical surface $h=r_{0}$. In analogy to the three-dimensional case [cf. Eq. (31)], we write

$$
\begin{aligned}
& h(z, t)=r_{0}[1+\epsilon \cosh (\omega t) \cos (k z)], \\
& v(z, t)=\epsilon v_{0} \sinh (\omega t) \sin (k z)
\end{aligned}
$$

and linearize in $\epsilon$. A calculation analogous to that of the full problem leads to the dispersion relation (34). As we have mentioned before, this dispersion relation accurately approximates the true growth rates for all $\omega$. Even more important, the stability boundary is at $k r_{0}=1$, the same as for the full equations. This means shortwavelength perturbations will not grow, in contrast to the leading-order equation (81), which produces infinite growth rates for the inviscid problem and still produces finite growth rates in the limit $k \rightarrow \infty$ for finite viscosity (Forest and Wang, 1990). Thus the leading-order system is unstable against very-short-wavelength "noise," making it ill suited for numerical purposes. If one includes higher-order terms perturbatively (Bechtel, Carlson, and Forest, 1995) the problem is not mended, since the leading-order equations are unaffected by higher-order ones.

Linear stability has also been investigated for various higher-order models such as the Cosserat equations (Bogy, 1979b) and the parabolic approximation (Garcia and Castellanos, 1994). The dispersion relation of these models still improves the comparison with Rayleigh's formula. For example, for the Cosserat equation the relative error in the growth rate is less than $0.25 \%$ for all unstable wave numbers.

Even using a system that is linearly stable against short-wavelength perturbations, numerical simulations of the fully nonlinear behavior are by no means trivial. This is true in particular in the neighborhood of the pinch point, which is characterized by very small length and time scales, and a blowup of the velocity. The asymptotic behavior near pinchoff described in the next subsection sets in only when $h_{\min } \leq \ell_{\nu} / 20=\nu^{2} \rho /(20 \gamma)$. Therefore, to simulate the motion of a drop of water falling from a $1 \mathrm{~cm}$ nozzle, one needs to resolve 8 orders of magnitude in the minimum height. Moreover, lowviscosity fluids are characterized by steep gradients, which have to be resolved carefully and are likely to cause numerical instabilities.

One can only hope to reach sufficient spatial resolution when the grid is locally refined near the singularity. Since the singularity moves in space and resolution requirements cannot be foreseen, the grid has to be ad- 
justed to meet certain accuracy requirements. To ensure sufficient temporal resolution, the time step has to be dynamically adjusted as well. Finally, a solution that incorporates spatial and temporal scales over many orders of magnitude, will also be prone to numerical instabilities. These have to be dealt with using implicit methods (Press et al., 1992).

The first fully nonlinear calculation is due to Lee (1974), who integrated the lubrication equations (79), (80), and (82) with zero viscosity and using periodic boundary conditions. A more extensive numerical study of the Cosserat equations (74), (75) in the inviscid and the viscous case was performed by Bogy, Shine, and Talke (1980). Like Lee, they used finite-difference equations, studied explicit as well as implicit methods, and discussed their stability. By contrast, Schulkes (1993) used finite-element methods to compare various inviscid models, namely, the full potential-flow equations (9)(11), the inviscid Cosserat equations, and Lee's model. He found considerable differences between the three cases, probably due to the fact that inviscid models become inconsistent even before breakup occurs, as shown by Eggers and Dupont (1994).

The first quantitative comparison of a onedimensional model with experiment was carried out by Eggers and Dupont (1994), who also included a detailed analysis of the pinch singularity. To do that, they used a second-order finite-difference scheme with a highly nonuniform mesh, which was adapted to the solution. The time integration was a fully implicit second-order scheme with adaptive step size control. It forced them to solve a nonlinear system of equations at each time step, which they did using Newton's method. A fully implicit scheme was found to be essential in order to control numerical instabilities. Figure 15 shows a comparison of the numerical computations by Eggers and Dupont with a drop of water at the bifurcation point. The photograph is the same as Fig. 10, in which the comparison is with a potential flow simulation.

The lubrication model correctly predicts the global shape, as it does the drop size, the length of the neck, and the profile near the bifurcation point. This only becomes possible if one goes beyond the asymptotic model, Eqs. (79)-(81), and includes the higher-order corrections, [Eq. (82)] in the curvature, such that spherical drops become an equilibrium shape. The model developed by Eggers and Dupont (1994) has also been used in an extensive study of the nonlinear evolution of a liquid bridge (Zhang, Padgett, and Basaran, 1996). Again, excellent agreement with experiment was found.

The numerical code originally developed by Eggers and Dupont has been subsequently refined in several directions. Shi, Brenner, and Nagel (1994) used a dynamically evolving grid, which introduced local refinement once specified conditions on the solution are met. In the case of low viscosities, treated by Brenner et al. (1997), special care had to be taken, since lack of viscous damping made the solution sensitive to perturbations. Moreover, steep gradients of the height and the velocity field occured even before $h_{\min }$ went to zero. These had

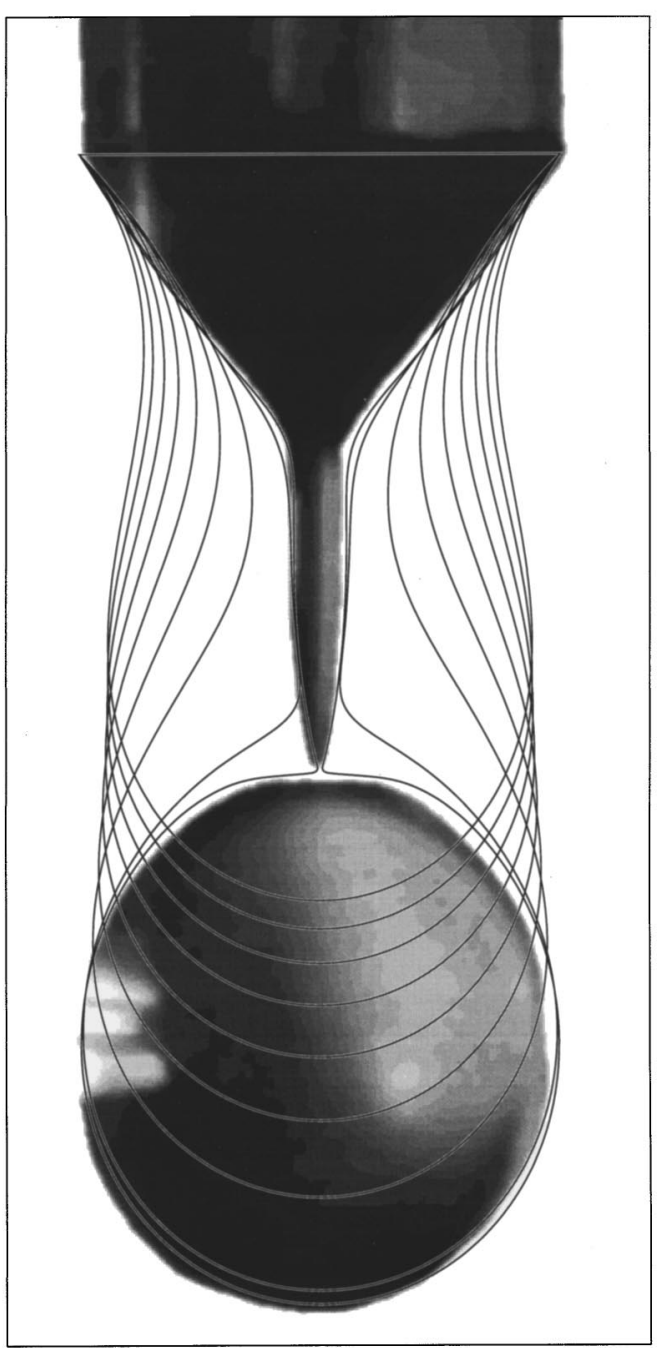

FIG. 15. The same photograph as in Fig. 10, compared with the lubrication equations of this section (Eggers and Dupont, 1994). The lines represent the profile at time distances of $0.4\left(r_{0}^{3} \rho / \gamma\right)^{1 / 2}$ and at the time of pinchoff.

to be dealt with by introducing additional refinement in regions of large gradients. The sensitivity to noise was accounted for by introducing a smooth mesh, whose grid sizes were continuously varied, as opposed to the grading in steps of two used by Eggers and Dupont. Through a combination of all of the above precautions, Brenner et al. (1997) dealt with a Reynolds number corresponding to a fluid $1 / 10$ of the viscosity of water, falling from a $1 \mathrm{~cm}$ nozzle. To fully resolve this problem, the solution had to be followed through 10 orders of magnitude in the minimum height, while the slope reached a maximum of 2000.

To be able to follow up on the evolution after breakup, Brenner et al. (1997) also developed a numerical scheme to cut the computational domain into two halves. Once some minimum thickness was reached, typically $10^{-3}$ times the initial thickness, the interface was extrapolated to a spherical cap on either side and additional grid points were introduced up to their tips. The two parts of the solution then evolved indepen- 


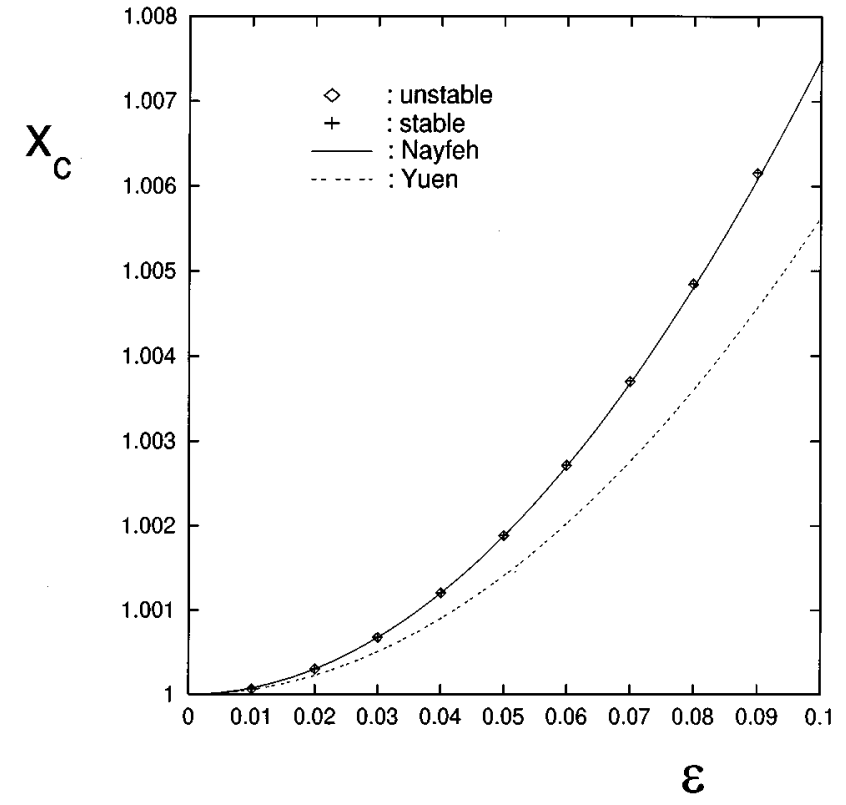

FIG. 16. Comparison between a numerical simulation of the one-dimensional lubrication equations and nonlinear stability theory. The stability boundary $x_{c}$ in the reduced wave number is plotted against the perturbation amplitude $\epsilon$. The numerical simulations clearly confirm Nayfeh's (1970) theory.

dently. By using different threshold thicknesses, they were able to confirm that the subsequent evolution was not affected by the details of the breakup on scales larger than the threshold thickness. Brenner et al. (1997) also tested the validity of the lubrication equations after breakup, by comparing numerical and experimental profiles before and after the bifurcation of a drop of water falling from a faucet. They found that excellent agreement persisted to times after breakup.

By using the high-resolution numerical codes, it is also interesting to repeat the nonlinear stability analysis of Sec. IV.C for the one-dimensional equations (Eggers, 1995b). The algebra is very similar, and in particular the nonlinear stability boundary (48) comes out to the same value, at least up to third order in perturbation theory. To test it numerically, a cylindrical column of fluid with periodic boundary conditions was set up, with a small sinusoidal perturbation imposed on it. As usual, all lengths were measured in units of the unperturbed radius. Thus the initial conditions were

$$
\begin{aligned}
& h(z, 0)=R-\epsilon \cos (x z), \\
& R=\left(1-\epsilon^{2} / 2\right)^{1 / 2},
\end{aligned}
$$

which conserves volume independent of $\epsilon$. The total length of the column was $0 \leqslant z \leqslant 2 \pi / x$, corresponding to one wavelength. The velocity was zero initially. Since Eq. (48) is only valid for inviscid flow, we applied the extremely large Reynolds number $\mathrm{Re}=10000$, so viscosity could be neglected. The perturbation amplitude was chosen to be between 0.01 and 0.1 , to make perturbation theory applicable. The resolution of 50000 grid points was selected to safely resolve wave numbers to $0.01 \%$.

Figure 16 shows the largest unstable wave number as diamonds and the smallest stable one as crosses. The resolution is so high that they cannot be distinguished on the scale of the plot. The full line is the prediction of Nayfeh's (1970) theory, the dashed line represents Yuen's (1968) theory. Lafrance's (1975) work would predict no shift in the critical wave number. Clearly, the validity of Nayfeh's theory near the cutoff is confirmed spectacularly. For $\epsilon \gtrsim 0.05$ theory systematically underestimates the cutoff value, a result, presumably, of higherorder effects in $\epsilon$.

\section{Inviscid theory and conservation laws}

In this subsection we shall discuss the fascinating connections of the one-dimensional equations with the theory of integrable systems (Hoppe, 1992). These connections are found most elegantly if the equations are transformed to a Langrangian frame of reference. Let $\beta$ be the position of a particle at time $t=0$ or, alternatively, and of a slice of fluid of width $d \beta$. Because the motion is one dimensional and volume is conserved, the same slice, after being convected to a position $z(\beta, t)$, will have the thickness $\left(h^{2}(\beta, 0) / h^{2}(z, t)\right) d \beta$. Thus we obtain

$$
z_{\beta}=\frac{h^{2}(\beta, 0)}{h^{2}(z(\beta, t), t)},
$$

where $z(\beta, t)$ is the position at time $t$ of the particle originally at $\beta$. Subscripts refer to differentiation with respect to the variable. By changing the labeling according to $d \alpha=h^{2}(\beta, 0) d \beta$, we can write Eq. (92) as

$$
z_{\alpha}=h^{-2}(z(\alpha, t), t)
$$

where $d \alpha$ is the volume of the slice $\alpha$. Equation (93) is thus a direct consequence of the volume of fluid elements being conserved. From the definition of $z$ we have

$$
z_{t}=v(z(\alpha, t), t) \text {. }
$$

Making use of the transformation $\partial_{z}=z_{\alpha}^{-1} \partial_{\alpha}$, Eqs. (93) and (94) allow us to rewrite the equation of motion, Eq. (79), in terms of $z(\alpha, t)$ alone:

$$
z_{t t}=-\frac{1}{\rho} \frac{\partial_{\alpha} p(z(\alpha, t))}{z_{\alpha}}+3 \nu z_{\alpha}\left(\frac{z_{t \alpha}}{z_{\alpha}}\right)_{\alpha} .
$$

The equation for $h$, Eq. (80), is automatically satisfied owing to the definition of $z(\alpha, t)$. If $p$ is replaced by its leading-order estimate (81), we have simply

$$
z_{t t}=-\frac{\gamma}{2 \rho} \frac{z_{\alpha \alpha}}{z_{\alpha}^{3 / 2}}+3 \nu\left(\frac{z_{t \alpha}}{z_{\alpha}^{2}}\right)_{\alpha} .
$$

Thus the coupled set of equations (79), (80) has been condensed into a single equation for $z(\alpha, t)$, which is of second order in time. If one leaves out the dissipation term, the equation becomes particularly simple:

$$
z_{t t}-\left(z_{\alpha}^{-1 / 2}\right)_{\alpha}=0
$$

where the constant $\gamma / \rho$ has been absorbed by a rescaling of variables. The same system has been studied extensively in a paper by Ting and Keller (1990), but in its original form [Eqs. (79)-(81)], with $\nu$ set equal to zero. 
We stress that Eq. (97) is unphysical as it stands. As we shall see in Sec. VII.B, the inviscid equations display singularities even before breakup occurs. Still, certain invariance properties of the inviscid system could reveal themselves even in the presence of a small amount of viscosity. In fact, we shall see below that Eq. (97) has an infinite sequence of independent conservation laws, apart from the obvious candidates of energy and mass. In the same vein, the Euler equation has been the subject of intense scrutiny (Majda, 1991), although it is believed to exhibit unphysical singularities of the vorticity (Grauer and Sideris, 1991; Pumir and Siggia, 1992). One hopes that the study of these singularities will yield information about high-Reynolds-number turbulence, in particular the mechanisms behind the energy cascade of eddies. Geometrical invariants of the Euler equation (Tur and Yanovsky, 1993) are expected to act as constraints for the almost inviscid motion of turbulent flow.

It is readily seen that Eq. (97) is generated by the Lagrangian density

$$
\mathcal{L}\left(z_{\alpha}, z_{t}\right)=z_{t}^{2} / 2+2 z_{\alpha}^{1 / 2} .
$$

The canonical momentum variable is $p=z_{t}$, so one finds for the Hamiltonian

$$
H=\int\left[\frac{p^{2}}{2}+V\left(z_{\alpha}\right)\right] d \alpha,
$$

where in our case we have

$$
V\left(z_{\alpha}\right)=2 z_{\alpha}^{1 / 2} \text {. }
$$

We have written the Hamiltonian in the general form (99), since the potential

$$
V\left(z_{\alpha}\right)=-A \frac{z_{\alpha}^{1-\gamma}}{1-\gamma}
$$

corresponds to the equations of one-dimensional compressible gas dynamics (Landau and Lifshitz, 1984a). These equations are usually written in the form

$$
\begin{aligned}
& \partial_{t} v+v v^{\prime}+\frac{p^{\prime}(\rho)}{\rho} \rho^{\prime}=0, \\
& \partial_{t} \rho+(v \rho)^{\prime}=0,
\end{aligned}
$$

where $p(\rho)=A \rho^{\gamma}$ is the pressure and $\rho$ the density of the gas. For the system to be stable one needs $A \gamma>0$, so that the pressure increases with density. A comparison between Eqs. (100) and (101) shows that $A=-1$ and $\gamma=1 / 2$, so the pinching problem corresponds to a new class of unstable systems, not considered in the realm of gas dynamics.

From among the many remarkable results (Olver and Nutku, 1988) that can be derived for Eq. (97), we mention that it can be linearized by means of the Legendre transformation

$$
\begin{aligned}
& \Phi\left(z_{\alpha}, z_{t}\right)=z-\alpha z_{\alpha}-t z_{t}, \\
& u=z_{\alpha}, \quad v=z_{t} .
\end{aligned}
$$

This is also known as a Hodograph transformation (Manwell, 1971). A straightforward calculation shows that Eq. (97) is now equivalent to the linear equation

$$
\Phi_{u u}+\frac{1}{2} \frac{\Phi_{v v}}{u^{3 / 2}}=0,
$$

which has an infinite sequence of exact solutions, as shown below (Olver and Nutku, 1988). So one possible approach is to transform the initial conditions of the original problem, Eq. (97), to the Hodograph plane $(u, v)$ and to solve Eq. (104) there.

Another, completely unexpected property of Eq. (104) is the following: Let $\mathcal{Q}(u, v)$ be a solution of Eq. (104). Then $\mathcal{Q}\left(z_{\alpha}, p\right)$ is a conserved density of the equations of motion. Conversely, all conserved quantities correspond to solutions of Eq. (104). For example, one can easily check that the Hamiltonian density

$$
\mathcal{H}(u, v)=\mathcal{Q}_{2}(u, v)=v^{2} / 2+2 u^{1 / 2}
$$

is a solution of the Hodograph equation (104). If one succeeds in finding other solutions of Eq. (104), other conservation laws in addition to the obvious ones of energy and momentum conservation will be generated. An infinite number of solutions are produced by the recursion relation (Hoppe, 1995)

$$
\begin{aligned}
& \partial_{v} \mathcal{Q}_{n+1}=n \mathcal{Q}_{n}, \\
& d_{\alpha}\left(\partial_{u} \mathcal{Q}_{n+1}\right)=n\left[v_{\alpha} \partial_{u} \mathcal{Q}_{n}-\frac{u_{\alpha}}{2 u^{3 / 2}} \partial_{v} \mathcal{Q}_{n}\right] .
\end{aligned}
$$

Starting from $\mathcal{Q}_{1}^{-}=u v$ and $\mathcal{Q}_{1}^{+}=v$ one generates two independent hierarchies of conservation laws, whose first members are

$$
\begin{aligned}
& \mathcal{Q}_{1}^{+}=v, \\
& \mathcal{Q}_{2}^{+}=v^{2} / 2+2 u^{1 / 2}, \\
& \mathcal{Q}_{3}^{+}=v^{3} / 3+4 v u^{1 / 2}, \\
& \mathcal{Q}_{4}^{+}=v^{4} / 4+6 v^{2} u^{1 / 2}-6 u \ln u,
\end{aligned}
$$

and

$$
\begin{aligned}
& \mathcal{Q}_{1}^{-}=u v, \\
& \mathcal{Q}_{2}^{-}=v^{2} u / 2-2 u^{3 / 2} / 3, \\
& \mathcal{Q}_{3}^{-}=v^{3} u / 3-4 v u^{3 / 2} / 3, \\
& \mathcal{Q}_{4}^{-}=v^{4} u / 4-2 v^{2} u^{3 / 2}+u^{2} .
\end{aligned}
$$

All the conserved quantities

$$
Q_{i}^{ \pm}=\int \mathcal{Q}_{i}^{ \pm} d \alpha
$$

are independent, i.e., they Poisson commute:

$$
\left\{Q_{i}^{ \pm}, Q_{j}^{ \pm}\right\}=0 \text {. }
$$

Note the emergence of logarithmic terms in Eq. (106), which appear in the fourth step of the hierarchy. This implies that the scale invariance

$$
\mathcal{Q}_{i}^{+}\left(a^{4} u, a v\right)=a^{i} \mathcal{Q}_{i}^{+}(u, v)
$$

of $\mathcal{Q}_{i}^{+}$is broken for $i \geqslant 4$. The same is thus true for the corresponding solutions $z(\alpha, t)$, since by virtue of the Hodograph transformation (103) each of the quantities 
TABLE I. The fluid parameters for water, glycerol, and mercury. The values are quoted from Weast (1978). The internal scales $\ell_{\nu}$ and $t_{\nu}$ are calculated from the kinematic viscosity $\nu$ and from the ratio of surface tension $\gamma$ and density $\rho$.

\begin{tabular}{lccc}
\hline \hline & Mercury & Water & Glycerol \\
\hline$\nu\left[\mathrm{cm}^{2} / \mathrm{s}\right]$ & 0.0012 & 0.01 & 11.8 \\
$\gamma / \rho\left[\mathrm{cm}^{3} / \mathrm{s}^{2}\right]$ fluid-air interface & 34.7 & 72.9 & 50.3 \\
$\ell_{\nu}=\nu^{2} \rho / \gamma[\mathrm{cm}]$ & $4.2 \times 10^{-8}$ & $1.38 \times 10^{-6}$ & 2.79 \\
$t_{\nu}=\nu^{3} \rho^{2} / \gamma^{2}[\mathrm{~s}]$ & $1.4 \times 10^{-12}$ & $1.91 \times 10^{-10}$ & 0.652 \\
\hline \hline
\end{tabular}

$\mathcal{Q}_{i}^{ \pm}$generates an exact solution of the nonlinear equation (97). For example, $\mathcal{Q}_{2}^{+}$corresponds to the solution

$$
z(\alpha, t)=z_{0}-t^{2} / 2-1 / \alpha .
$$

In contrast to Eq. (109), the solution derived from $\mathcal{Q}_{4}^{+}$ will no longer be scale invariant but contains logarithmic terms, which is a telltale sign of anomalous scaling (Amit, 1978). Unfortunately, Eq. (109) results in a height $h(z, t)$ which has a singularity at a finite $z$ for all times. In fact, it is a remarkable consequence of the infinite sequence of conservation laws, Eqs. (106), (107), that no localized pinching solution of the inviscid equations can exist. As we shall explain in more detail in Sec. VII.B, a conserved quantity of sufficiently high order would always diverge during pinching, which is inconsistent with regular initial data. Thus it is of fundamental importance to work in the effect of small amounts of viscosity, which will eventually destroy the rigorous conservation properties. On large scales, on the other hand, the motion is still subject to the constraints imposed by Eqs. (106), (107), as viscosity is not yet important. The limit of small viscosities has also been used to study shock-wave solutions of inviscid equations, for example in the case of the equations of one-dimensional elasticity (Dafermos, 1987). It is likely that Eq. (97) would develop infinitely sharp gradients as well.

\section{SIMILARITY SOLUTIONS AND BREAKUP}

\section{A. Local similarity form}

Experimental observations have revealed that freesurface shapes of a given fluid are remarkably similar near the pinch point, independent of initial conditions. For example, jet breakup at different wavelengths (Fig. 4) and under the influence of gravity (Fig. 6) all show a conical tip attached to a sharp front if the fluid is water. For high viscosities (Figs. 5 and 7) the solutions turn out to be threads. So while the outer dimension $L$ of the experimental setup (say the radius of the nozzle) hardly seems to matter, there is a strong dependence on viscosity. In fact, if $L$ does not enter the description near breakup, the only other scale the solution can depend on is the "inner" length scale $\ell_{\nu}=\nu^{2} \rho / \gamma$ and the corresponding time scale $t_{\nu}=\nu^{3} \rho^{2} / \gamma^{2}$. Thus, when doubling the viscosity, breakup should look similar when viewed on length scales four times as large and time scales eight times as long as already noted by Haenlein (1931).

Some values of $\ell_{\nu}$ and $t_{\nu}$ are shown in Table I for three common Newtonian fluids. Variations in $\ell_{\nu}$ and $t_{\nu}$ are huge, which is an asset for experiments: just by choosing different fluids, one can explore a wide range of different scaling behaviors. For water, $\ell_{\nu}$ is almost on molecular scales, while for glycerol it is about the same as typical outer scales. Since the relevant scale of pinching solutions is set by $\ell_{\nu}$ and $t_{\nu}$, none of the asymptotics can be seen in the experiments with water, at least at the present state of spatial and temporal resolution. It is rather the long and skinny threads observed in highviscosity experiments that are characteristic of the final stages of pinching.

The solutions close to the pinch point, which we assume to be at $z_{0}$ and at a time $t_{0}$, depend only on $\ell_{\nu}$ and $t_{\nu}$. Thus the height and the velocity should be representable as

$$
\begin{aligned}
& h(z, t)=\ell_{\nu} h_{s}\left(z^{\prime}, t^{\prime}\right), \\
& v(z, t)=\frac{\ell_{v}}{t_{\nu}} v_{s}\left(z^{\prime}, t^{\prime}\right),
\end{aligned}
$$

where $z^{\prime}=\left(z-z_{0}\right) / \ell_{\nu}$ and $t^{\prime}=\left(t_{0}-t\right) / t_{\nu}$ measure the distance from the singularity and $h_{s}, v_{s}$ are universal functions. This universality was verified numerically using the lubrication equations (79), (80), and (82) for different viscosities and initial conditions (Eggers and Dupont, 1994).

Near a singularity the flow lacks a typical scale, so self-similarity is a natural concept. Indeed, a self-similar solution of a flow leading to a singularity was already given by Guderley (1942) in the case of a spherical shock wave. Constantin et al. (1993) and Bertozzi et al. (1994) studied the breakup of a fluid drop between two closely spaced glass plates using similarity solutions. In the context of the present problem, Ting and Keller (1990) studied singular solutions of an inviscid version of the lubrication equations (79)-(81). Similarity solutions of the viscous equations were found by Eggers (1993).

The existence of self-similar solutions of the pinching problem is in fact already implicit in the scaling analysis of Sec. V.A. Equation (50) means that the balance of inertial, surface-tension, and viscous forces is independent of the parameter $\epsilon$, which measures the minimum thickness of the thread. Thus the leading-order equations (59), (60), and (62) must allow for scale-invariant solutions that obey Eq. (50). If $\tau$ of Sec. V.A is identified as the time distance to the singularity, there exist solutions of the form 


$$
\begin{aligned}
& h_{s}\left(z^{\prime}, t^{\prime}\right)=\left|t^{\prime}\right| \phi(\xi), \\
& v_{s}\left(z^{\prime}, t^{\prime}\right)=\left|t^{\prime}\right|^{-1 / 2} \psi(\xi), \\
& \xi=z^{\prime} /\left|t^{\prime}\right|^{1 / 2} .
\end{aligned}
$$

The scaling ansatz (111) implies that the typical radius of the fluid scales like $\tau$, and the axial extension like $\tau^{1 / 2}$, just as is required by Eq. (50). The coordinates $z^{\prime}$ and $t^{\prime}$ have been nondimensionalized with $\ell_{\nu}$ and $t_{\nu}$. If $\left|t^{\prime}\right| \ll 1$, this means that $\epsilon$ is small according to Eq. (50), so one is in the asymptotic limit where higher-order corrections to the leading-order equations can be neglected. In this sense $\ell_{\nu}$ and $t_{\nu}$ measure the size of the region where asymptotic self-similarity can be expected.

Plugging the ansatz (111) into the leading-order equations, one confirms that it is a solution, provided the similarity functions $\phi$ and $\psi$ obey the ordinary differential equations

$$
\begin{aligned}
& \pm\left(\psi / 2+\xi \psi^{\prime} / 2\right)+\psi \psi^{\prime}=\phi^{\prime} / \phi^{2}+3\left(\psi^{\prime} \phi^{2}\right)^{\prime} / \phi^{2}, \\
& \pm\left(-\phi+\xi \phi^{\prime} / 2\right)+\psi \phi^{\prime}=-\psi^{\prime} \phi / 2 .
\end{aligned}
$$

The plus sign refers to the time before breakup, where $t<t_{0}$, while the minus sign refers to the time after breakup. For this solution, forces blow up like $\left|t^{\prime}\right|^{-3 / 2}$, so any constant external force such as gravity, becomes negligible. Other higher-order corrections to Eq. (111) coming from the Navier-Stokes equation are smaller by at least a factor of $\left|t^{\prime}\right|$, so they vanish in the limit of $t^{\prime} \rightarrow 0$. It follows that near the pinch point the complete Navier-Stokes problem can be reduced enormously and simplified to a pair of ordinary differential equations. The main task is to solve Eqs. (112) and (113) before and after the singularity, and to investigate how these solutions can be matched onto the outer solution far away from breakup.

\section{B. Before breakup}

Before breakup $t$ is smaller than $t_{0}$, and the + in Eqs. (112) and (113) applies. At first it seems as if there would be a plethora of possible solutions, since the system (112), (113) is of third order, so the manifold of solutions is three dimensional. But looking back at Eq. (111) one discovers that the asymptotic behavior of $\phi$ and $\psi$ for large $\xi$ is severely restricted, since $h_{s}$ and $v_{s}$ must remain finite at any fixed distance from the singularity as $t^{\prime} \rightarrow 0$. Otherwise one would have to move a finite amount of fluid with arbitrarily high speed. Since any finite $z^{\prime}$ expands to infinity in similarity variables, this translates into a condition on $\phi$ and $\psi$ at infinity: for $\xi \rightarrow \pm \infty$ one must have

$$
\begin{aligned}
& \phi(\xi) / \xi^{2} \rightarrow a_{0}^{ \pm}, \\
& \psi(\xi) \xi \rightarrow b_{0}^{ \pm},
\end{aligned}
$$

where $a_{0}^{ \pm}$and $b_{0}^{ \pm}$are constants. The condition (114) is a particular example of a "matching condition," which guarantees that the "inner solution" close to $z_{0}, t_{0}$ is consistent with an "outer solution" that moves on much longer length and time scales. In most cases, such as the
Blasius boundary layer (Landau and Lifshitz, 1984a), the inner solution will depend on constants such as $a_{0}^{ \pm}$and $b_{0}^{ \pm}$. They are set by solving the outer problem with proper regard of the boundary conditions. Remarkably, in the present problem the situation is reversed: the constants are determined by the inner problem, and the outer solution must be matched onto it. On the other hand, a certain freedom is hidden in the way of representing the inner solution. The coordinates $z_{0}, t_{0}$ will of course depend on the outer solution, and can only be found a priori by solving the complete problem including boundary conditions.

To investigate the limit $\xi \rightarrow \pm \infty$ in more detail, we try an asymptotic expansion of $\phi$ and $\psi$ consistent with (114):

$$
\begin{aligned}
& \phi(\xi)=\xi^{2} \sum_{i=0}^{\infty} a_{i} \xi^{-2 i} \\
& \psi(\xi)=\frac{1}{\xi} \sum_{i=0}^{\infty} b_{i} \xi^{-2 i} .
\end{aligned}
$$

By inserting Eq. (115) into (112), (113), one finds all the coefficients recursively in terms of $a_{0}$ and $b_{0}$ (Eggers, 1995a). This is surprising at first, because Eqs. (112), (113) are a third-order system, so one would expect three free parameters in the expansion instead of just $a_{0}$ and $b_{0}$. This indicates that Eq. (115) represents only a certain submanifold of solutions. What the other solutions look like, at least in the neighborhood of Eq. (115), can be found by perturbing around (115). Thus we write

$$
\begin{aligned}
& \phi(\xi)=\phi_{0}(\xi)+\epsilon_{1}(\xi), \\
& \psi(\xi)=\psi_{0}(\xi)+\epsilon_{2}(\xi),
\end{aligned}
$$

where $\phi_{0}, \psi_{0}$ is a solution of the form (115), and linearize Eqs. (112) and (113) in $\epsilon_{1}$ and $\epsilon_{2}$. One obtains a linear system with nonconstant coefficients, whose solution can be found by a WKB-type analysis (Ting and Keller, 1990). This means one writes $\epsilon_{1}, \epsilon_{2}$ in the form

$$
\begin{aligned}
& \epsilon_{1}(\xi)=\exp [\chi(\xi)] \sum_{i=0}^{\infty} f_{i} \xi^{\nu-2 i} \\
& \epsilon_{2}(\xi)=\exp [\chi(\xi)] \sum_{i=0}^{\infty} g_{i} \xi^{\mu-2 i} .
\end{aligned}
$$

Upon inserting this ansatz into the linearized equations one can determine the free exponents $\nu$ and $\mu$ and in particular the exponential

$$
\chi(\xi)=\xi^{2} / 12 \text {. }
$$

Thus solutions close to the two-dimensional manifold (115) deviate exponentially from it as one integrates outward to plus or minus infinity. Given $a_{0}$ and $b_{0}$, there is one more free coefficient $f_{0}$ contained in Eq. (117); all the other $f_{i}$ and $g_{i}$ are determined from it recursively. Thus $f_{0}$ carries the missing third degree of freedom not contained in Eq. (115).

The important point to keep in mind from this analysis is that for a solution to be consistent with the 

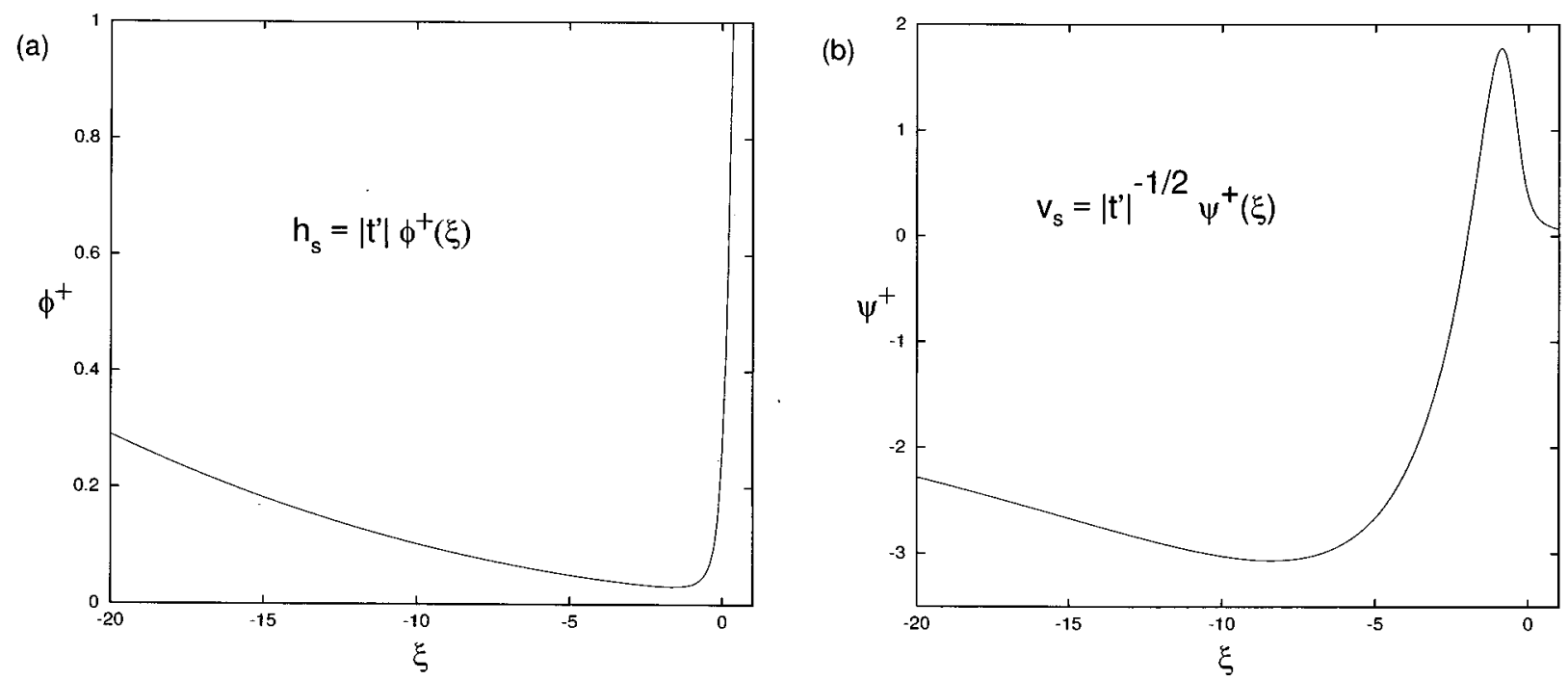

FIG. 17. The similarity functions $\phi^{+}$and $\psi^{+}$before breakup. Note the strong asymmetry.

asymptotic behavior, Eq. (114), it must lie on a twodimensional submanifold parametrized by $a_{0}^{+}, b_{0}^{+}$for $\xi \rightarrow \infty$ and by $a_{0}^{-}, b_{0}^{-}$for $\xi \rightarrow-\infty$. All other solutions deviate exponentially from the correct behavior. Thus, even without specifying $a_{0}^{ \pm}$and $b_{0}^{ \pm}$, the asymptotic behavior (114) amounts to one algebraic constraint on the solution at $+\infty$ and one at $-\infty$. There is only a onedimensional manifold of solutions consistent with this. The remaining degree of freedom is eliminated by considering the structure of singularities in the interior of the domain. Rewriting Eq. (113) in the form

$$
\phi^{\prime}=\phi \frac{1-\psi^{\prime} / 2}{\psi+\xi / 2},
$$

one discovers that there must be a point $\xi_{0}$ with

$$
\psi\left(\xi_{0}\right)+\xi_{0} / 2=0 .
$$

Thus the interface will have a singularity unless the condition

$$
\psi^{\prime}\left(\xi_{0}\right)=2
$$

is also met. A closer analysis reveals that this is a movable singularity with a simple pole in $\psi$ and an algebraic branch point in $\phi$, which disappears with the condition (119). Clearly, the velocity cannot be infinite at finite $t^{\prime}$, so one has to restrict oneself to a regular expansion ,

$$
\begin{aligned}
& \phi(\xi)=\sum_{i=0}^{\infty} \phi_{i}\left(\xi-\xi_{0}\right)^{i}, \\
& \psi(\xi)=\sum_{i=0}^{\infty} \psi_{i}\left(\xi-\xi_{0}\right)^{i} .
\end{aligned}
$$

Except for $\xi_{0}$, the only free parameter in this expansion is $\phi_{0}=\phi\left(\xi_{0}\right)$; all other $\phi_{i}$ and $\psi_{i}$ follow from it (Eggers, 1995a). Analyzing the higher orders of this expansion, one finds a finite radius of convergence. Thus the procedure for finding a solution consistent with all the conditions is the following: One starts with a pair of values $\left(\xi_{0}, \phi_{0}\right)$, from which the solution can be integrated outwards to $+\infty$ and $-\infty$. Generically the solution will not be consistent with Eq. (114), but only a one-dimensional submanifold of $\left(\xi_{0}, \phi_{0}\right)$ will lead to the correct asymptotic behavior at $+\infty$ and $-\infty$, respectively. The points where they cross correspond to physical solutions of Eqs. (112), (113). Such a solution was found by Eggers (1993) and compared with numerical simulations (Eggers and Dupont, 1994). The corresponding similarity functions are plotted in Fig. 17, and some characteristic parameters are given in Table II. Recently, additional solutions consistent with the physical conditions were discovered (Brenner, Lister, and Stone, 1996). One of these additional solutions, which probably form an infinite sequence, is also listed in Table II. All correspond to much smaller $\phi_{0}$, and thus to thinner threads than the solution of Fig. 17. Using the stability analysis of the next subsection, Brenner, Lister, and Stone (1996) have shown that the additional solutions are extremely

TABLE II. Some characteristics of the similarity functions $\phi^{+}, \psi^{+}$before breakup. The first row corresponds to the functions represented in Fig. 17, the second row to the most stable of the additional solutions found by Brenner, Lister, and Stone (1996). The symbol $\xi_{0}$ stands for the position of the stagnation point, where the fluid is at rest in the frame of reference of the interface. The minimum value of $\phi^{+}$is $\phi_{\min }$. The function $\psi^{+}$reaches a maximum value of $\psi_{\max }$. The numbers $a_{0}^{ \pm}$stand

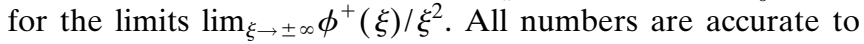
the decimal places shown.

\begin{tabular}{lcccc}
\hline \hline$\xi_{0}$ & $\phi_{\min }$ & $\psi_{\max }$ & $a_{0}^{+}$ & $a_{0}^{-}$ \\
\hline-1.5699 & 0.030426 & -3.066 & 4.635 & $6.047 \times 10^{-4}$ \\
-1.8140 & 0.010785 & -4.698 & 52.75 & $\approx 5 . \times 10^{-4}$ \\
\hline \hline
\end{tabular}



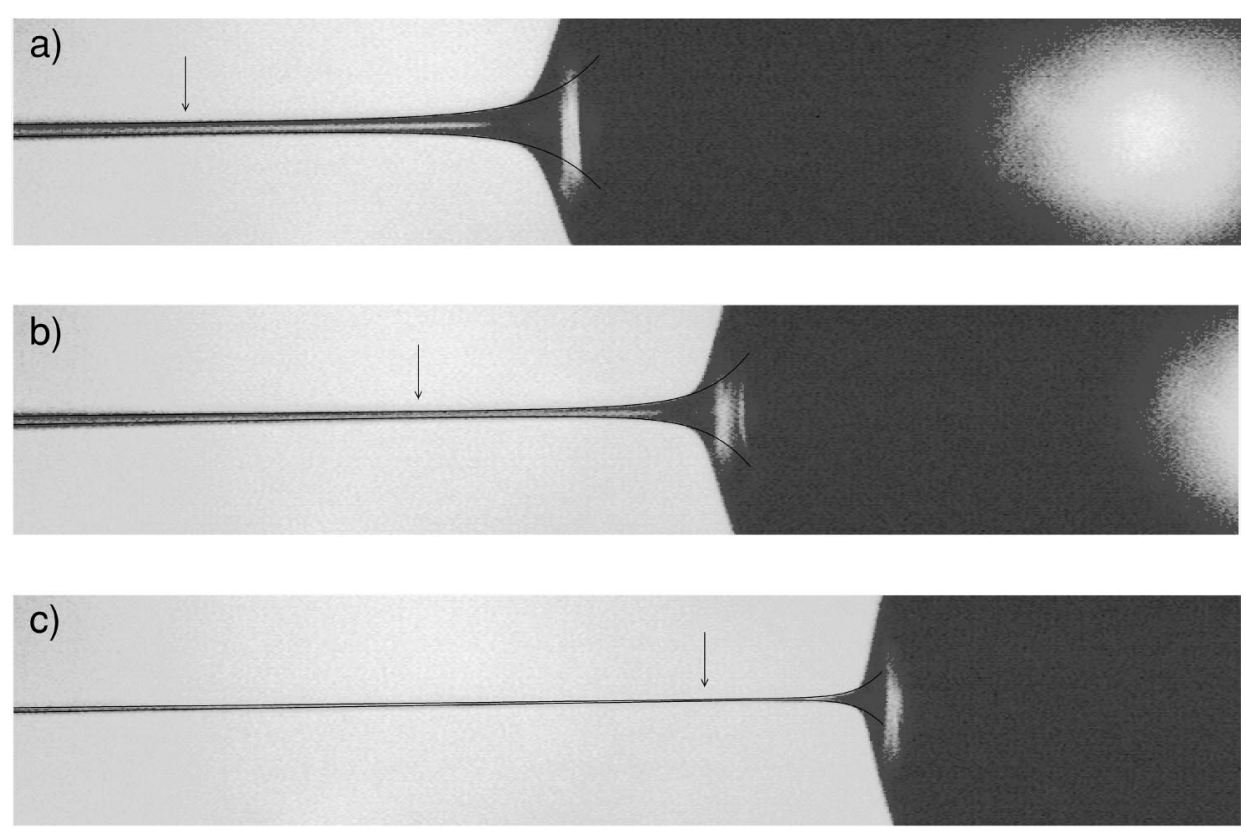

FIG. 18. A sequence of closeups of a highly viscous jet $\left(\ell_{\nu}=274.5 \mu \mathrm{m}, t_{\nu}=627.9 \mu \mathrm{s}\right)$ $350 \mu \mathrm{s}, 198 \mu \mathrm{s}$, and $46 \mu \mathrm{s}$ away from breakup. The frame width corresponds to $1 \mathrm{~mm}$. The full line is the prediction of the scaling theory of Eq. (111) with scaling function $\phi^{+}$of Fig. 17. Each frame has been cut at top and bottom, so only a small portion of the drop forming on the right is seen. The pictures are from the series of experiments GLY1 (see Table 1 of Kowalewski, 1996). unstable, so the only one of physical relevance is the one with the largest $\phi_{0}$, originally found by Eggers (1993).

Note that using this pair of similarity functions, the full solution near the pinch point can be predicted without adjustable parameters. For example, at a given time $t_{0}-t=t_{\nu}\left|t^{\prime}\right|$ away from the singularity, the minimum diameter of the fluid thread is $0.06 \ell_{\nu}\left|t^{\prime}\right|$. So if $t_{0}-t$ is measured experimentally, the entire profile close to the singularity can be predicted without adjustable parameters. This was done in a recent series of jet experiments with high-viscosity fluids (Kowalewski, 1996).

Figure 18 shows a closeup of the pinch region for a jet consisting of a mixture of glycerol and alcohol at times 350,198 , and $46 \mu$ s before breakup, which corresponds to the reduced times $\left|t^{\prime}\right|=0.56,0.32$, and 0.073 . The jet diameter at the nozzle is $0.397 \mathrm{~mm}$, which is about a third of the image width. When considering the thin thread, one is looking at a part of the solution that is moving on scales very different from the one set by the undisturbed jet. The experimental pictures have been superimposed with the theoretical prediction. Since $t^{\prime}$ is known, there is no adjustable parameter in this comparison. The theoretical prediction for the position of the minimum is marked by an arrow, which is seen to move toward the drop adjacent to the thread. In (a), the solution has only started to converge on its left, so there is some discrepancy in the position of the minimum. As time goes on, the range of validity of the similarity solution expands.

One immediately notices the asymmetry of the solution, which is already apparent from the scaling functions (see Fig. 17). The flatness of the neck is much exaggerated by the scaling of Eq. (111), which expands axial scales relative to the radius. As the slope becomes of order one near the drop, the similarity solution no longer applies. To extend the prediction beyond the range of validity of the similarity solution, one would have to find an outer solution that described the drop and to match it onto the inner solution. In so doing, one has to remember that the outer solution will depend on all the experimental details such as the nozzle diameter, the fluid parameters, and the driving mechanism. On the other hand, the pinching solution is independent of these details, as confirmed by Kowalewski (1996) when he performed experiments with different nozzle diameters and fluid viscosities.

However, as the thread becomes thinner, a new and unexpected behavior sets in, shown in Fig. 19 for a fluid of higher viscosity. The interface assumes an irregular appearance with bulges on its surface. Apparently, the similarity solution has become unstable in its interior. This instability was first described experimentally by Shi, Brenner, and Nagel (1994), who offered a theoretical explanation in Brenner, Shi, and Nagel (1994). This work will be presented in the next subsection.

\section{Stability and the influence of noise}

It is apparent from Fig. 19 that the similarity solution is prone to the growth of localized perturbations or "bumps." It seems as if this should be expected from the stability analysis of a liquid cylinder, which the solution very much resembles as it is becoming thinner. In fact, it follows from the high-viscosity limits (35) and (36) that the typical wavelength of a bump will be $\lambda_{\text {bump }} \sim r_{0}\left(\ell_{\nu} / r_{0}\right)^{1 / 4}$, while the time scale over which it grows is $t_{\text {bump }} \sim t_{\nu} r_{0} / \ell_{\nu}$, so it is localized on the scale of the thread length and grows over the same time scale on which the solution is evolving. The reason the similarity solution is still observed is that the flow in the thread has a stabilizing effect: perturbations are both stretched and convected out of the pinch region, where they no longer grow. Only perturbations that are sufficiently large initially will affect the similarity solution. This leads to a nonlinear instability, quite similar to the fingering insta- 


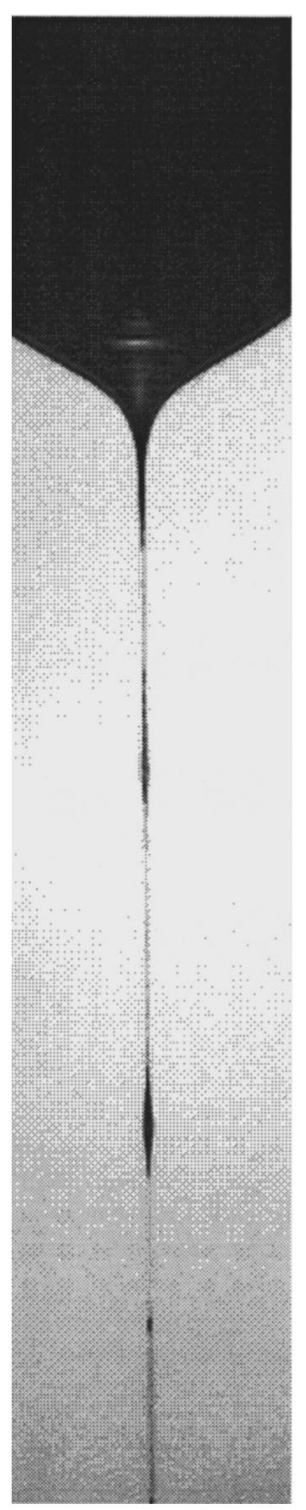

FIG. 19. The same experiment as in Fig. 18, but with a fluid of higher viscosity, $\left(\ell_{\nu}=2 \mathrm{~mm}, t_{\nu}=12.8 \mathrm{~ms}\right.$, series GLY3 of Kowalewski, 1996). The height of the frame corresponds to $2 \mathrm{~mm}$. Just before breakup, the thin thread has become unstable and produced several "bumps." The moment of breakup shows irregular breakup at several places.

bility in Hele-Shaw flow (Bensimon et al., 1986), or dendritic growth (Kessler et al., 1988).

For an analytical study of the stability problem, such as was presented by Brenner, Shi, and Nagel (1994), one takes advantage of the perturbations being localized. Thus it is sufficient to keep track of the center of mass and the characteristic width of a localized wave packet. Close to the similarity solution one can work within the framework of the asymptotic equations (79), (80). However, one has to keep some of the higher-order terms in the mean curvature in Eq. (82), since they are responsible for selecting the characteristic size of the bump. By rewriting the equations in terms of the logarithmic time distance $s=\ln \left|t^{\prime}\right|$ from the singularity and the similarity variable $\xi$, one is working on the scale of the similarity solution. This leads to the ansatz

$$
\begin{aligned}
& h_{s}\left(z^{\prime}, t^{\prime}\right)=\left|t^{\prime}\right|(\phi(\xi)+\bar{\phi}(\xi, s)), \\
& v_{s}\left(z^{\prime}, t^{\prime}\right)=\left|t^{\prime}\right|^{-1 / 2}(\psi(\xi)+\bar{\psi}(\xi, s)),
\end{aligned}
$$

where $\phi(\xi)$ and $\psi(\xi)$ are the solutions to the similarity equation described in the previous subsection, and $\bar{\phi}$, $\bar{\psi}$ are small perturbations to it. Using a prime to indicate differentiation with respect to $\xi$, one has the linearized equations of motion

$$
\begin{aligned}
\partial_{s} \bar{\phi}(\xi, s)= & \bar{\phi}-\left(\psi+\frac{\xi}{2}\right) \bar{\phi}^{\prime}-\phi^{\prime} \bar{\psi}-\frac{\psi^{\prime}}{2} \bar{\phi}-\frac{\phi}{2} \bar{\psi}^{\prime}, \\
\partial_{s} \bar{\psi}(\xi, s)= & -\frac{\bar{\psi}}{2}-\left(\psi+\frac{\xi}{2}\right) \bar{\psi}^{\prime}-\psi^{\prime} \bar{\psi}+\frac{\bar{\phi}^{\prime}}{\phi^{2}}-2 \frac{\phi^{\prime}}{\phi^{3}} \bar{\phi} \\
& +3 \bar{\psi}^{\prime \prime}+6 \frac{\phi^{\prime}}{\phi} \bar{\psi}^{\prime}+6 \frac{\psi^{\prime}}{\phi} \bar{\phi}^{\prime}-6 \frac{\psi^{\prime} \phi^{\prime}}{\phi^{2}} \bar{\phi}+t^{\prime} \bar{\phi}^{\prime \prime \prime} .
\end{aligned}
$$

From the higher-order terms of the mean curvature we have included only the highest derivative of the perturbation, since it is the one which limits the wavelength from below.

Solutions of the linear problem (122) have the form

$$
\left(\begin{array}{c}
\bar{\phi} \\
\bar{\psi}
\end{array}\right)=\left(\begin{array}{c}
\cos \vartheta \\
\sin \vartheta
\end{array}\right) \exp \{S(\zeta, \xi, s)\},
$$

where the exponential describes the growth of perturbations. By having $S$ depend on $\xi$ and $\zeta=\xi / \epsilon$, one separates the short-wavelength perturbation, which moves on the scale of $\zeta$, from the slowly varying "background" of the similarity solution. The ansatz

$S(\zeta, \xi, s)=\frac{1}{\epsilon} \beta_{0}(\kappa(s), \xi) s+i \kappa(s) \zeta+\beta_{1}(\kappa(s), \xi) s$

describes a perturbation of wavelength $\epsilon / \kappa(s)$, which is convected with velocity $\beta_{0} / \kappa(s)$ in similarity variables, and whose amplitude grows with rate $\beta_{1}$. Indeed, plugging Eqs. (123), (124) into the linearized equations, one find to leading order $\epsilon^{-1}$

$$
\beta_{0}=-i \kappa(s)(\psi+\xi / 2)
$$

and

$$
\partial_{s} \kappa=-\kappa(s)\left(\psi^{\prime}+\frac{1}{2}\right) .
$$

This means a bump is convected with the interfacial velocity

$$
\psi_{i}=\psi+\xi / 2,
$$

which is the velocity of the fluid in the frame of reference of the surface. At the stagnation point $\xi_{0}$ [see Eq. (118)], the bump always remains on the same point of the surface. At the same time, the bump is stretched with rate $-\left(\psi^{\prime}+1 / 2\right)$, which is negative in the neck region (see Fig. 17). Hence the wavelength increases, making the disturbance more benign.

The temporal growth rate is 


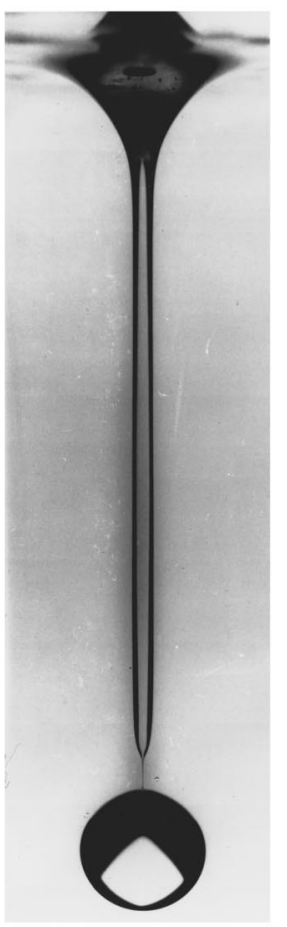

(a)

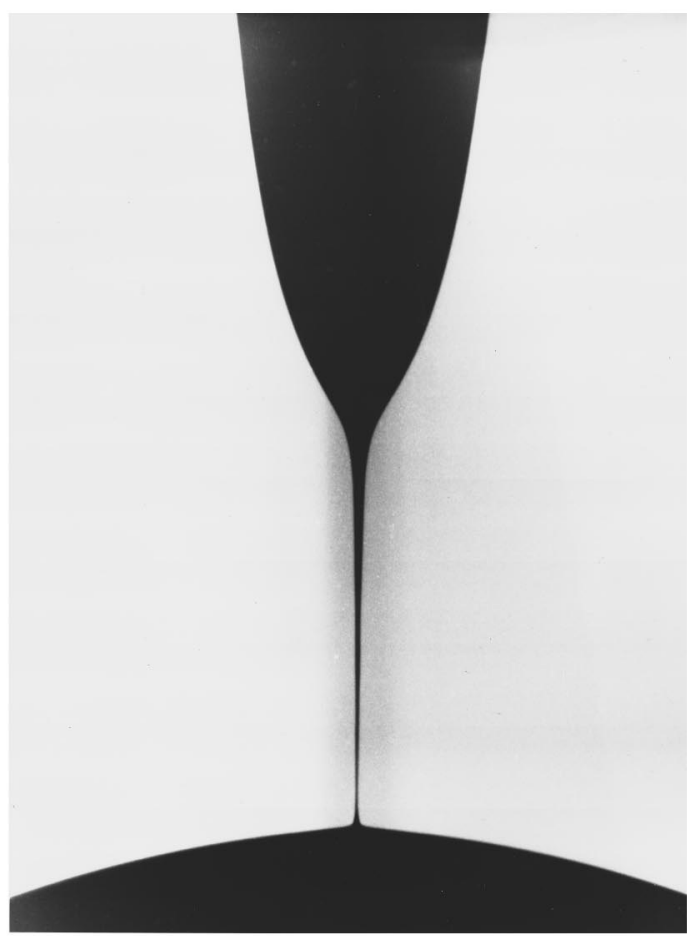

(b)

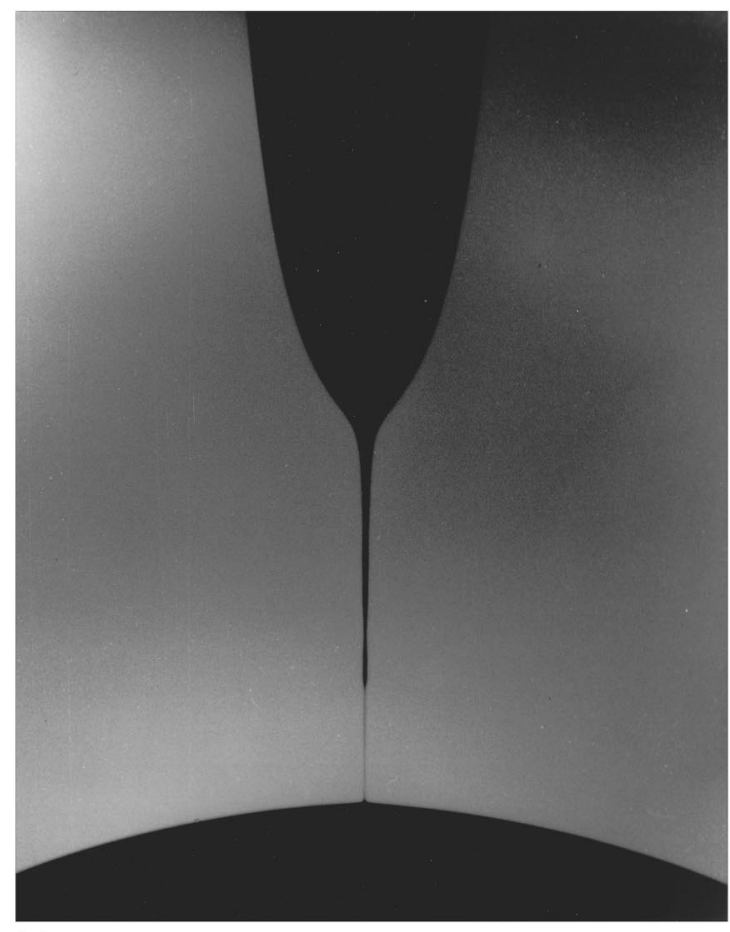

(c)

FIG. 20. A photograph of a glycerol in water mixture (85 w\%) (Shi, Brenner, and Nagel, 1994). Part (a) is an overall view showing a tiny microthread growing out of the initial neck, (b) is a magnified view of the "micro-thread," which is close to a similarity solution $2 \times 10^{-3} \mathrm{~s}$ away from breakup. Image (c) shows the same neck as in (b), but at a later time. One observes an even smaller neck growing out of the original micro-thread, which is again a similarity solution with the estimated breakup time of $2 \times 10^{-4} \mathrm{~s}$. Reprinted with permission. (C) American Association for the Advancement of Science.

$$
\beta_{1}(\kappa, \xi)=1+\frac{\psi^{\prime}}{2}+\frac{1}{6 \phi}\left[1-t^{\prime}\left(\phi \frac{\kappa}{\epsilon}\right)^{2}\right]
$$

which can be translated into a spatial growth rate by $\psi_{i}$,

$$
\frac{\partial_{\xi} A}{A(\xi)}=\frac{\beta_{1}}{\psi_{i}},
$$

where $A(\xi)$ is the amplitude of a bump at $\xi$. If $A_{0}=A(\bar{\xi})$ is the initial amplitude at a reference point $\bar{\xi}$, Eq. (128) can be solved to give

$$
\frac{A(\xi)}{A_{0}}=\exp \left\{\int_{\bar{\xi}}^{\xi} \frac{\xi \beta_{1}\left(\xi^{\prime}\right)}{\psi_{i}\left(\xi^{\prime}\right)} d \xi^{\prime}\right\} .
$$

From this it seems as if arbitrary growth could be obtained for $\bar{\xi}=\xi_{0}$, leaving a bump at the same point forever. But that would require an arbitrarily sharp spike, while a bump with positive growth rate must have approximate size $t^{\prime 1 / 2}$ according to Eq. (127). Thus $\bar{\xi} \approx t^{\prime 1 / 2}$ should be taken as the lower limit in Eq. (129). On the other hand, an analysis of Eq. (129) for large $|\xi|$ reveals, using $\phi=a_{0}^{ \pm} \xi^{2}$ and $\psi=b_{0}^{ \pm} \xi^{-1}$, that a bump no longer grows for $\xi \rightarrow \pm \infty$. Such a bump, which is frozen in on the time scale of the singularity, has been called a "blob." When one uses the parameters of the similarity solution, any bump that gets convected to $\xi_{-} \approx-10$ or $\xi_{+} \approx 1.5$ meets such a fate.

This completes the input necessary to estimate the critical amplitude that makes the similarity solution unstable. The amplitude $A(\xi)$ has to grow to the same size as the similarity solution itself before it reaches $\xi_{-}$or $\xi_{+}$, starting from $\bar{\xi} \approx t^{\prime 1 / 2}$. Using this condition, Brenner, Shi, and Nagel (1994) estimated the critical amplitude for surface perturbations to be

$$
A_{c}=2 \times 10^{-5} t^{\prime 1.495}
$$

in units of the minimum thickness. In real space this means that for a thread of radius $h_{0}$ one needs a surface perturbation of size

$$
h_{c}=3.8 \times 10^{-3} h_{0}\left(\frac{h_{0}}{\ell_{\nu}}\right)^{1.495}
$$

to render the interface unstable. Thus in the late stages of singularity formation, for which $h_{0} \ll \ell_{\nu}$, the solution becomes more and more vulnerable to external perturbations, in qualitative agreement with experiment. Figure 20 shows a picture of a mixture of glycerol and water falling from a nozzle. Part (b) is a magnification of the thin thread in (a), which is close to the similarity solution, $2 \mathrm{~ms}$ away from breakup. In part (c) this solution has undergone an instability, and a smaller neck has grown on it, which is again well approximated by a similarity solution, $200 \mu$ s away from breakup.

This conclusion is amplified by a series of numerical simulations of the asymptotic model (79), (80), and (82) with some stochastic noise added to it. Figure 21 shows a 

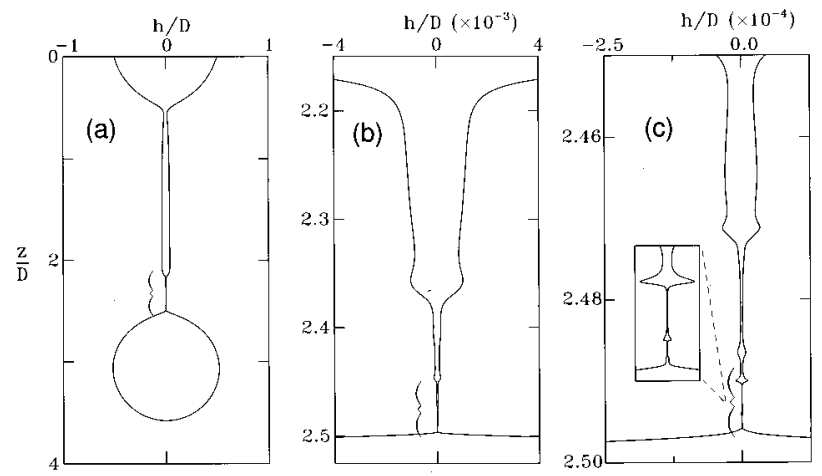

FIG. 21. Simulation of a drop of viscous fluid for the same physical parameters as in Fig. 20 (Shi, Brenner, and Nagel, 1994). The left frame (a) shows the same view as in Fig. 20(a), while (b) is an enlargement of the micro-thread region, simulated in the presence of noise. In addition to the neck seen in Fig. 20(c), which has grown out of the original micro-thread, one sees another stage of the sequence of instabilities. Part (c) suggests that this sequence of instabilities goes on indefinitely, by showing further enlargements of the unstable region.

simulation with the same parameters as in the experiment (see Fig. 20). The first picture (a) shows the same thread as was in the experimental picture. Without noise, it evolves according to the similarity solution up to breakup. However, once noise is added, one observes [see Fig. 21(b)] tiny necks growing on the original similarity solution, similar to Fig. 20(c). In the simulation one can follow up on this solution, and observe still another neck growing on that of the previous stage. Interestingly, one confirms that each of those necks represents another similarity solution, but with a different value of $z_{0}$ and $t_{0}$. If numerical resolution is increased, this cascade of similarity solutions can be followed through an arbitrary number of instabilities, Fig. 21(c), going down to the stage of the seventh neck. The above stability analysis has been repeated by Brenner, Lister, and Stone (1996) for the additional similarity solutions found by them, one of which is listed in Table II. For these solutions, the analogue of the critical amplitude (130) is much smaller, so they immediately go unstable and converge to the similarity solution described above, even if care is taken to reduce the noise as much as possible.

The main open problem is the source of the noise that causes the instability. A possibility discussed by Brenner, Shi, and Nagel (1994) is thermal capillary waves, whose amplitude can be estimated using the theory of hydrodynamic fluctuations (Landau and Lifshitz, 1984a). The result is that thermal fluctuations become important when the minimum radius becomes of the order of

$$
h_{\text {thres }}=5 \ell_{\nu}\left(\frac{\ell_{T}}{\ell_{\nu}}\right)^{0.401},
$$

where

$$
\ell_{T}=\left(k_{B} T / \gamma\right)^{1 / 2}
$$

is the thermal length scale. For the fluid of Fig. 20, this gives $h_{\text {thres }}=1 \mu \mathrm{m}$, which rather underestimates the scale where irregular behavior is observed in experiment. On the other hand, in the jet experiment of Fig. 18 one finds $h_{\text {thres }}=10 \mu \mathrm{m}$, while the smallest radius in Fig. 18 is about $1 \mu \mathrm{m}$. Clearly, more carefully controlled experiments are needed to quantitatively identify possible sources of the noise.

Finally, we mention that a comparison with the stability analysis of Sec. IV.A implies that the axisymmetric singularity will be stable against nonaxisymmetric disturbances. Strictly speaking, the singularity could still be nonaxisymmetric if the initial condition deviates greatly from axisymmetry. On the other hand, a spherical cross section corresponds to a global minimum in surface area, with oscillations strongly damped by viscosity, so it is hard to see how nonaxisymmetry could persist. Another possible departure from the proposed universal solution could lie in a nonzero azimuthal velocity field, driven through the nonlinear coupling of the NavierStokes equation. Both possibilities are worthwhile subjects of theoretical and experimental research.

\section{After breakup}

Having investigated the structure of solutions up to the point of breakup, one finds it natural to ask what the behavior will be after breakup. At a scale $\ell_{\text {micro }}$ the interface will be broken by some microscopic mechanism, whose nature cannot be investigated within the framework of hydrodynamics. One possible guess is that this scale is on the order of intermolecular distances. In the molecular dynamics calculations of Koplik and Banavar (1993), pinching of threads ten molecules in diameter was observed. Although the behavior was reminiscent of hydrodynamic behavior, no quantitative comparison was attempted, so the questions of mechanisms of rupture and up to what scale to apply hydrodynamics are really open in the case of free surfaces. At high viscosities, the instability described in the previous subsection will make the interface look "fuzzy" and ever more susceptible to (multiple) breakings. The scale at which a drop breaks can expected to be much larger than molecular distances if the viscosity is large. Whatever the scale may be, the fundamental problem is whether the subsequent evolution depends on the microscopic aspects of the breaking on scales much larger than $\ell_{\text {micro }}$. If it is independent, the mathematical equivalent is that there is a unique prescription to continue the equations of motion beyond the singularity.

Questions of this type have been investigated extensively on a mathematical level (Altschuler, Angenent, and Giga, 1995). This was done mostly for geometrical evolution problems like that of mean curvature flow (Huisken, 1991; Deckelnick, 1997), but also for the nonlinear Schrödinger equation (Giga and Kohn, 1989; Merle, 1992), for which the continuation is not unique. The reason is that the complex wave function can pick up an arbitrary phase after breakup. The problem of a liquid bridge is very different because it includes inertia. This prevents fluid away from the breakup point from 


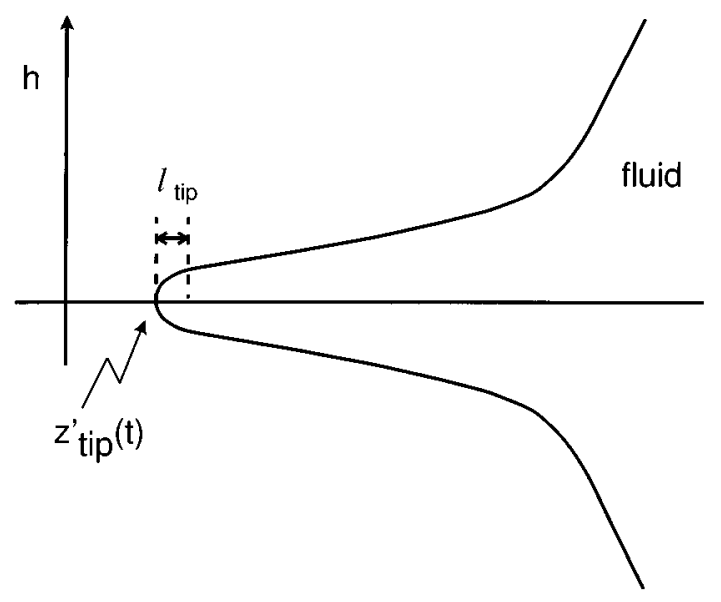

FIG. 22. A cartoon of a receding tip after breakup. The size of the tip region $\ell_{\text {tip }}$ is the same as the typical width of the neck.

moving much on the time scale of the breaking, which indicates that the solution (now consisting of two parts) should indeed be independent of the mechanisms of breakup. The most direct way to show this is, of course, to construct explicitly a solution in a similar vein to that in which similarity solutions were found before breakup. Similarity solutions that correspond to a unique continuation through a singular point in time were constructed for the porous-medium equation by Grundy (1983), and for the shallow-water equations by Grundy and Bell (1993). Keller and Miksis (1983) have constructed similarity solutions of an inviscid wedge of fluid after breakup. The asymptotics far away from the point of breakup enter the solution as a free parameter. In our case the asymptotic behavior is already known: it should coincide with the solution before breakup, so the parameters are uniquely determined.

Figure 22 shows a schematic view of a receding neck after breakup. The same asymptotic equations as before breakup apply, since shortly after breakup the solution will still be a thin thread. However, the situation is different in the immediate neighborhood of the tip, where $h^{\prime}$ diverges and thus renders Eqs. (79) and (80) inapplicable. In fact, as $h \rightarrow 0$ and $h^{\prime} \rightarrow \infty$ near the tip, both the viscous term $\left(h^{2} v^{\prime}\right)^{\prime} / h^{2}$ and the asymptotic form of the pressure gradient $-h^{\prime} / h^{2}$ diverge. Thus one is faced with a boundary-layer problem: the thin tip region obeys a different type of scaling than the outer region, and therefore other equations apply. Both inner and outer solutions have to be accommodated by matching conditions. To establish the proper scaling, one observes that in the frame of reference of the receding tip the end is almost static, so it will approach a spherical equilibrium shape on a microscopic time scale. This means the size of the tip region $\ell_{\text {tip }}$ is the same as its radial extension. Since the tip has to be matched onto the outer solution, both radial scales must coincide, and we have $\ell_{\text {tip }} \approx \ell_{\nu}\left|t^{\prime}\right|$, using the scaling of the outer solution. This means that in the scaling limit $t^{\prime} \rightarrow 0$ the size of the tip region goes to zero even in similarity variables. Hence the similarity equations (112), (113) can be applied up to the position of the tip

$$
\xi_{\text {tip }}=z_{\text {tip }}^{\prime}\left|t^{\prime}\right|^{1 / 2}
$$

in the limit $t^{\prime} \rightarrow 0$.

In his early study of inviscid receding threads Keller (1983) went beyond this asymptotic limit. There and in the somewhat more explicit treatment by Keller, King, and Ting (1995) both parts of the solution were considered, which scale differently. Using mass and momentum balances, as well as matching conditions, approximate solutions for the tip region and the neck were found. By contrast, Eggers (1995a), using the full viscous theory, considered only the limit $t^{\prime} \rightarrow 0$ in which the tip region shrinks to zero. In that case, a unique answer for the shape of the neck was obtained. The only property of the tip solution needed was that all terms of the equation remain finite as one approached the tip, the underlying assumption being that the exact form of the tip solution does not matter.

The idea behind the solution proposed by Eggers (1995a) was to supplement the asymptotic equations (79)-(81) with higher-order terms, which remove the singularity at the tip. The solution of the regularized equations is thus valid at the tip and can be analyzed in the limit $\left|t^{\prime}\right| \rightarrow 0$. This leads to a unique answer for the asymptotic form of the tip, independent of the regularization chosen. To produce a regular tip, one can generalize the equations to the form (Constantin, 1992)

$$
\begin{aligned}
& \partial_{t} h+v h^{\prime}=-\frac{v^{\prime} h}{2}, \\
& \partial_{t} v+v v^{\prime}=-p^{\prime} / \rho+\frac{\left(v^{\prime} D^{2}\right)^{\prime}}{h^{2}},
\end{aligned}
$$

where

$$
p=\frac{1}{2 h}\left[\frac{\partial E}{\partial h}-\left(\frac{\partial E}{\partial h^{\prime}}\right)^{\prime}\right] .
$$

Here $E\left(h, h^{\prime}\right)$ is a surface energy and $D\left(h, h^{\prime}\right)$ a dissipation kernel. Equation (134) can also be rewritten in the form

$$
\partial_{t} v+v v^{\prime}=\frac{1}{h^{2}}\left[-G / \rho+v^{\prime} D^{2}\right],
$$

where

$$
G\left(h, h^{\prime}\right)=h^{2} p+h^{\prime} \frac{\partial E}{\partial h^{\prime}}-E .
$$

From this one immediately derives the energy conservation equation

$$
\partial_{t}\left[h^{2} v^{2} / 2+E\left(h, h^{\prime}\right)\right]=-\left(v^{\prime} D\right)^{2}+\text { boundary terms, }
$$

which motivates the nomenclature for $E$ and $D$. As far as the asymptotic analysis is concerned, the only information needed from $G$ and $D$ appearing in Eq. (135) is that they vanish at the tip. This is a direct consequence 

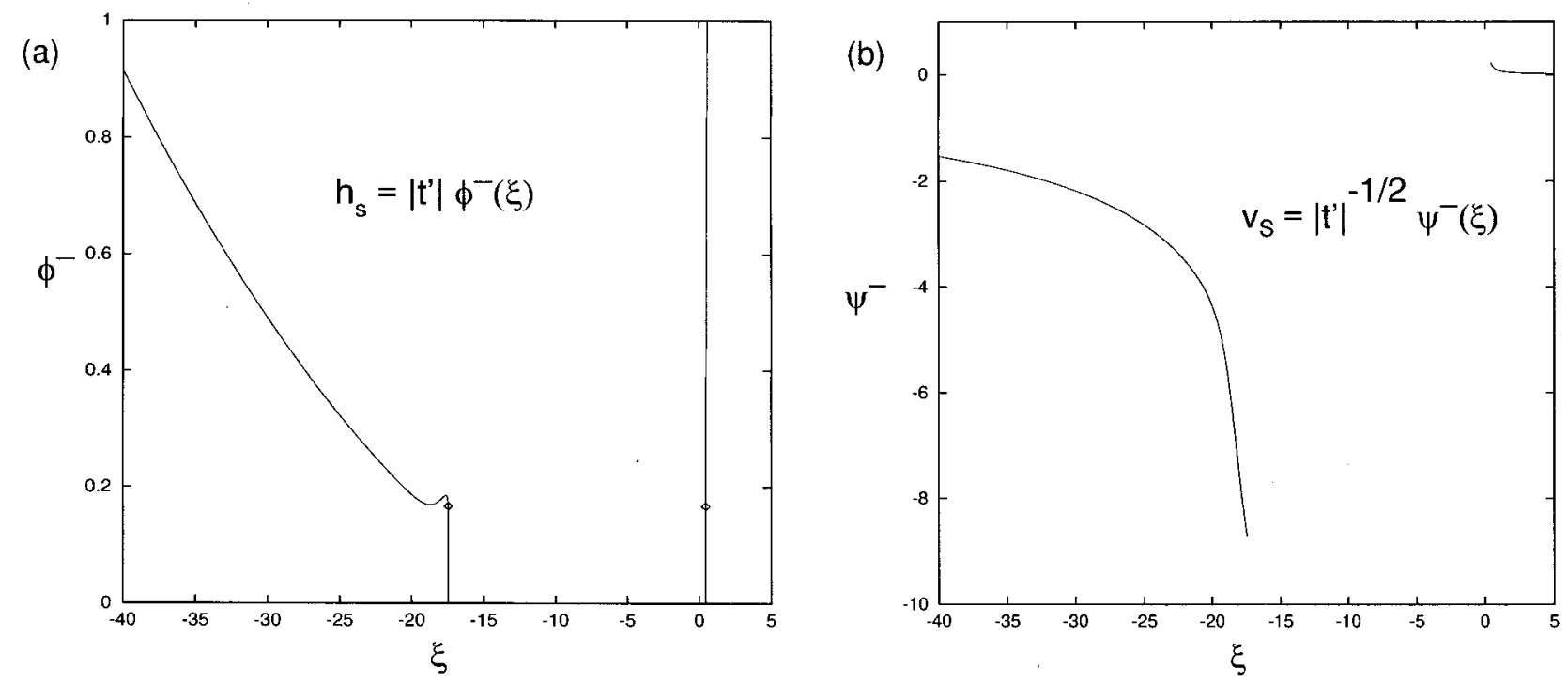

FIG. 23. The similarity functions $\phi^{-}$and $\psi^{-}$. The asymptotic behavior for $\xi \rightarrow \pm \infty$ is the same as before breakup. On the left side is the rapidly receding "neck" part of the solution, on the other side is the drop. The points $\xi_{\text {neck }}$ and $\xi_{\text {drop }}$, from where the interface is plane, are marked by diamonds.

of the pressure gradient and the dissipation term's being finite near a regular tip $h \sim\left(z-z_{\text {tip }}\right)^{1 / 2}$.

The equations of motion (133) and (135) need only be analyzed in a small region of size $\ell_{\text {tip }}$, where the fluid has to move with the receding tip. Therefore, in coordinates moving with the tip position, the inertial terms drop out. In terms of similarity variables, the transformation to the comoving frame reads

$$
\begin{aligned}
& \bar{\phi}(\bar{\xi})=\phi\left(\bar{\xi}+\xi_{\text {tip }}\right), \\
& \bar{\psi}(\bar{\xi})=\psi\left(\bar{\xi}+\xi_{\text {tip }}\right)-\xi_{\text {tip }} / 2, \\
& \bar{\xi}=\xi-\xi_{\text {tip }} .
\end{aligned}
$$

Plugging the similarity ansatz (111) into the equations of motion, one finds the similarity equations

$$
\begin{aligned}
& \bar{\phi}=-\bar{\psi}^{\prime} \bar{\phi} / 2, \\
& {\left[-\bar{G}+\bar{\psi}^{\prime} \bar{D}^{2}\right]^{\prime}=0}
\end{aligned}
$$

in the tip region of size $\bar{\xi} \approx\left|t^{\prime}\right|^{1 / 2}$. As remarked above, $\bar{G}$ and $\bar{D}$ are zero at the tip, so Eq. (139) can actually be written

$$
\bar{G}=\bar{\psi}^{\prime} \bar{D}^{2} .
$$

At $\bar{\xi} \approx\left|t^{\prime}\right|^{1 / 2}$, the asymptotic form of the equations become valid, and comparing Eqs. (139) and (112) we can write

$$
\bar{G}_{\text {asymp }}=-\bar{\phi}, \quad \bar{D}_{\text {asymp }}=\sqrt{3} \bar{\phi} .
$$

Thus from Eqs. (138), (140), and (141) one finds that $\bar{\phi}=1 / 6$, so the correct boundary condition for $\phi$ at the tip is

$$
\phi\left(\xi_{\text {tip }}\right)=1 / 6
$$

Now we can solve the similarity equations (112), (113) with the boundary condition (142). Note that after the singularity the minus sign applies. The solution near the tip has the expansion

$$
\begin{aligned}
& \phi=1 / 6+\phi_{1}\left(\xi-\xi_{\text {tip }}\right)^{2 / 5}+\cdots, \\
& \psi=\xi_{\text {tip }} / 2-2\left(\xi-\xi_{\text {tip }}\right)+e_{0}\left(\xi-\xi_{\text {tip }}\right)^{7 / 5}+\cdots .
\end{aligned}
$$

All the coefficients can be calculated from the two parameters $\phi_{1}$ and $\xi_{\text {tip }}$, which still need to be determined. Because of the change of sign in the equations, one now has exponential convergence onto the solutions with the asymptotics (114), as one integrates outwards to $\xi \rightarrow \pm \infty$. The constants $a_{0}, b_{0}$ are open and depend on $\phi_{1}, \xi_{\text {tip }}$. The solution is thus uniquely fixed by requiring that the constants $a_{0}, b_{0}$ before and after the singularity coincide. This is because the solution far outside the singularity is unaffected by the breaking. The resulting similarity functions are plotted in Fig. 23, while some of the characteristic parameters are given in Table III. The solution now consists of two parts, one representing a receding thread, the other just a small perturbation of a drop. Although obtained by the same procedure, they look very different, as the constants $a_{0}$ and $b_{0}$ are very different to the left and right of the singularity.

Figure 24 shows a particular example of how the solution before breakup is uniquely continued. After

TABLE III. Characteristics of the similarity functions $\phi^{-}, \psi^{-}$ after breakup. The tip position of the left-hand, or neck side is $\xi_{\text {neck }}$, and the expansion coefficient $\phi_{1}$ [cf. Eq. (143)], is $\phi_{\text {neck }}$. Correspondingly, $\xi_{\text {drop }}$ and $\phi_{\text {drop }}$ uniquely determine the "drop" side of $\phi^{-}$and $\psi^{-}$. The values of $a_{0}^{ \pm}$and $b_{0}^{ \pm}$are the same as before breakup (see Table II).

\begin{tabular}{lccc}
\hline \hline$\xi_{\text {neck }}$ & $\phi_{\text {neck }}$ & $\xi_{\text {drop }}$ & $\phi_{\text {drop }}$ \\
\hline 17.452 & 0.06183 & 0.4476 & 0.6180 \\
\hline \hline
\end{tabular}




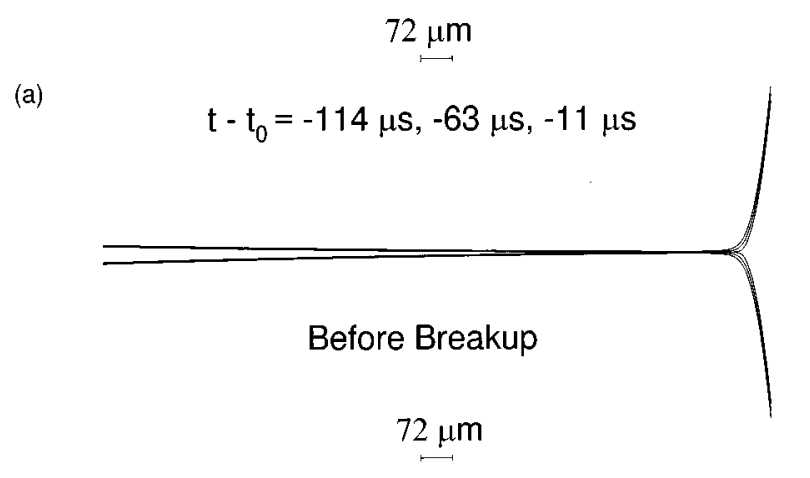

(b)

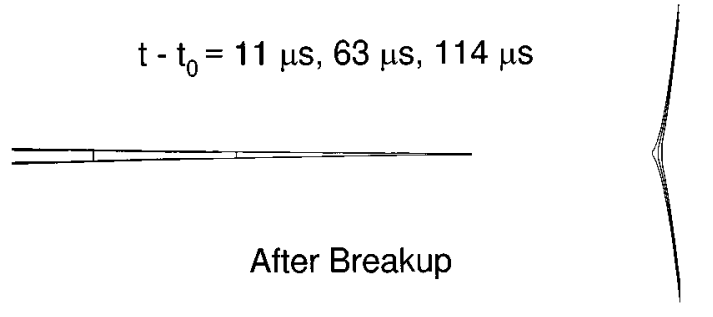

FIG. 24. The breakup of a mixture of glycerol in four parts of ethanol, as calculated from the similarity solutions. Part (a) shows three profiles before breakup, in time distances of 46 $\mu \mathrm{s}$, corresponding to $\left|t^{\prime}\right|=1,0.55$, and 0.1 . In part (b) the same is shown after breakup.

breakup, one part of the solution consists of a rapidly receding thread, the position of the tip being

$$
z_{\text {tip }}-z_{0}=\ell_{\nu} \xi_{\text {neck }} t^{\prime 1 / 2} \text {. }
$$

Thus the tip velocity goes like $\left|t^{\prime}\right|^{-1 / 2}$, making it slower as $t^{\prime}$ gets larger. The other part of the solution is visible as a small protuberance, which rapidly heals off to an almost flat interface.

Detailed comparisons of the similarity solution after breakup with numerical simulations of Eqs. (133) and (134) are reported in Eggers (1995a). This is documented in Fig. 25, which shows the approach of the similarity solution by a sequence of profiles calculated from Eqs. (133), (134), immediately after the interface had been cut. It was verified in (Eggers, 1995a) that this similarity solution is independent of the particular regularization used in Eq. (133). Thus the continuation indeed turns out to be unique, independent of the detail of the dynamics near the tip.

As far as comparison with experiment is concerned, the situation is less fortunate. Figure 26 shows the continuation of the series of pictures presented in Fig. 18. Qualitatively, the solution looks as predicted. But while the comparison with similarity theory is fully quantitative before breakup, two problems arise after breakup: First, there is some residue left between the two parts of the solution, which is seen as two tiny drops. This is because there is some unsteadiness in the solution before breakup, as can be seen in Fig. 26(a), which makes the thread break in more than one place. Second, the retraction speed is slower than is predicted by theory. In Fig. 26(b) we have estimated the time distance from

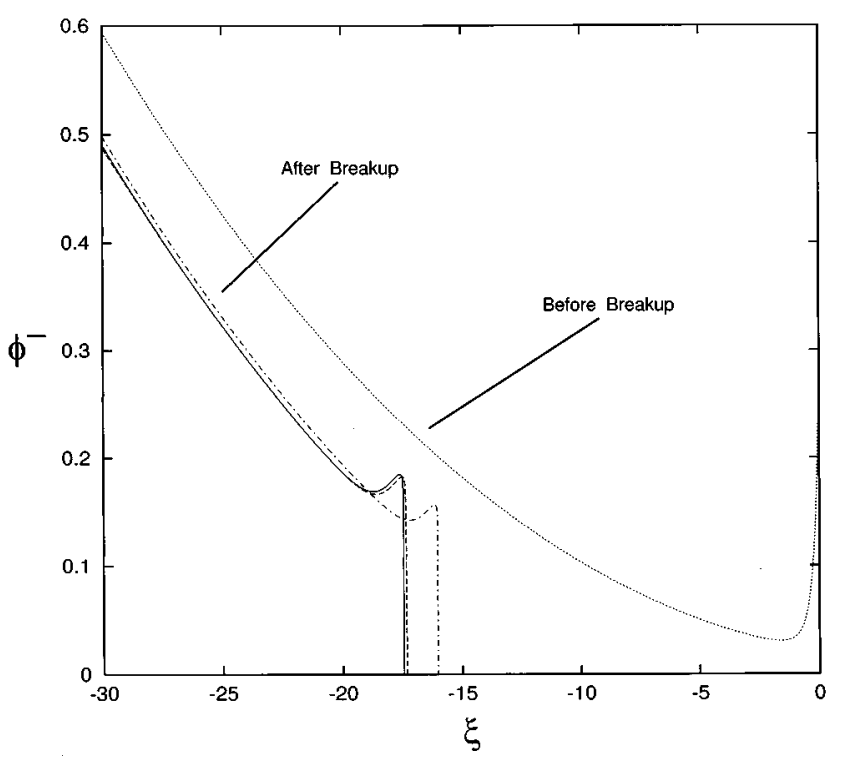

FIG. 25. The approach of the similarity function $\phi^{-}$by the solution of Eqs. (133) and (134) in the jet geometry, transformed to similarity variables. The fluid neck is severed at $\left|t^{\prime}\right|=10^{-4}$ before breakup. The full line is $\phi^{-}$, the dotted line the solution before breakup. The dot-dashed and the dashed lines show the simulation at $\left|t^{\prime}\right|=0.006$ and $\left|t^{\prime}\right|=0.06$, respectively.

breakup to be $6.7 \mu \mathrm{s}$, based on the position of the tip according to the similarity theory. The drop part of the solution is well represented by the similarity solution, while at the end of the thread some additional fluid has accumulated, probably a result of blobs formed in the irregular breakup. The distance the tip has retracted in Fig. 26(c) is only $80 \%$ of that predicted by theory on the basis of the timing of Fig. 26(b). The reason is probably the air drag on the tiny thread, which according to the similarity solution should have a speed of $37 \mathrm{~m} / \mathrm{s}$ and a thickness of $0.6 \mu \mathrm{m}$ in Fig. 26(b). While slender-body theory predicts the force due to air drag to be almost independent of the thread radius (Happel and Brenner, 1983), the force of retraction diminishes with the cross section. Thus the thread is very sensitive at small diameters. Close to breakup one therefore expects the tip to speed up rather than to slow down. Examples of this behavior have been observed by Kowalewski (1996), using the "frame-transfer" technique. Observations in qualitative agreement with Kowalewski's have also been made by Henderson, Pritchard, and Smolka (1997). To be able to make a quantitative comparison one must consider two possible approaches: The first is to include the outer fluid. Such a theory is being developed by Lister and Stone (1996). The other approach is to reduce the air drag as much as possible in order to observe directly the similarity solution. Some experiments in this direction were performed by Kowalewski (1996) in an evacuated chamber, using an oil of high vapor pressure. However, a comparison with theory is difficult since the breakup was quite unsteady in this particular series of experiments. It would be preferable to work with fluids 

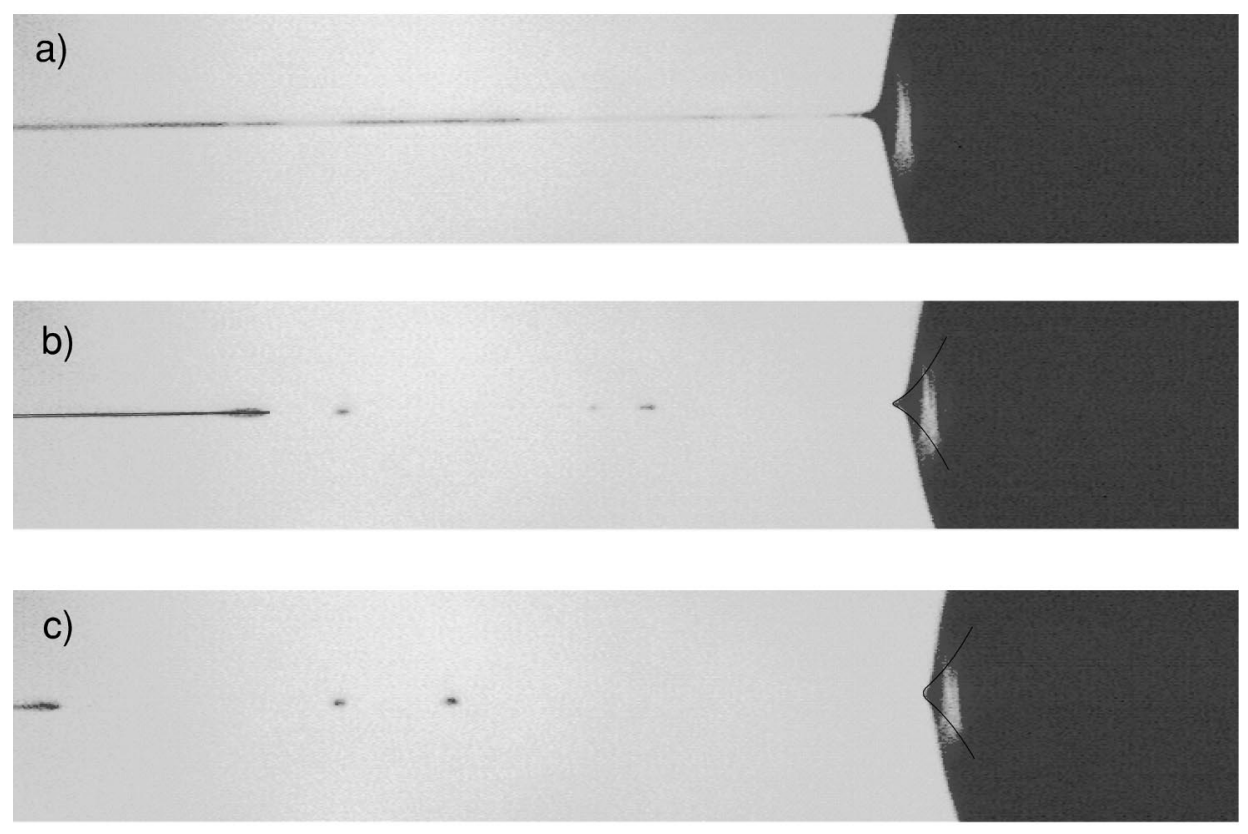

FIG. 26. Continuation of the sequence of Fig. 18, $6.7 \mu$ s before breakup, as well as $6.7 \mu \mathrm{s}$ and $22 \mu \mathrm{s}$ after breakup. The full line is the prediction of the similarity theory. The timing of (b) was estimated on the basis of this solution. of somewhat lower viscosity, making the solution less sensitive to noise.

\section{AWAY FROM BREAKUP}

\section{A. High viscosity-threads}

A familiar feature of the breakup of high-viscosity liquids is their tendency to produce very long and thin threads. As seen in the previous section, threads always occur in the last stages of pinching. But the most dramatic effects occur when the Reynolds number is already small, based on the initial extension and velocity of the fluid, and when threads are drawn by some external pull. This way, $\mu \mathrm{m}$ diameter threads can easily be extended to the length of several $\mathrm{cm}$. This is known not only from breakfast table experiments, but also from important industrial processes like fiber spinning. An example is shown in Fig. 27, in which gravitational pull is responsible for producing a thread of glycerol approximately $2 \mathrm{~cm}$ long.

According to Rayleigh's theory, the time scale on which disturbances grow increases with viscosity, but this can by no means account for the persistence of threads at such small diameters. In fact, the time scale over which a thread should become unstable is $t_{\nu}=r_{0} \rho \nu / \gamma$. Even for a fluid of such high viscosity as glycerol, but with a thread radius of $10 \mu \mathrm{m}$, the time scale is $t_{\nu}=230 \mu \mathrm{s}$, which is much shorter than the time over which the thread is pulled out in Fig. 27. So, clearly, the flow inside the thread has to be taken into account, just as it was in the stability analysis of the Sec. VI.C. So far, a quantitative explanation for the stability of threads in these more general circumstances has not been offered. Pearson and Matovich (1969) looked at the stability of an extending liquid thread, but did not include surface tension, which is the source of the potentially most dangerous instability. By analogy to the analysis of the previous section, both convection and a positive stretching rate will be important. Disturbances are convected out of regions where the thread is thinnest, and stretching pulls disturbances apart, lowering their amplitude. Most likely, the conditions under which a thread is stable are much more general than the specific case of a similarity solution. This remains an extremely interesting problem to be studied in more detail.

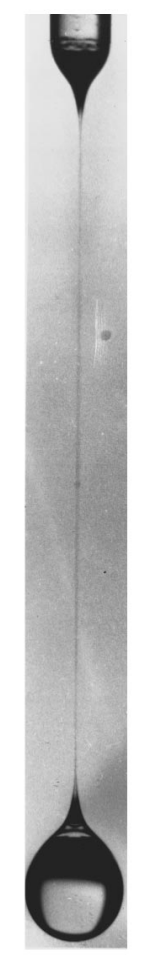

FIG. 27. A drop of glycerol falling from a nozzle $1.5 \mathrm{~mm}$ in diameter, pulling out a very long thread (Shi, Brenner, and Nagel, 1994). Reprinted with permission. (C) American Association for the Advancement of Science. 
Even without gravitational pull, threads can extend to the size of the external scale if the Reynolds number is sufficiently small. Evidence is provided by the pictures of jets and liquid bridges at high viscosities (see Figs. 5 and 8). Here it is surface tension itself that pulls out the threads and collects all the fluid in drops. The length of the thread is set by the wavelength of the perturbation and, as we shall see below, it thins almost uniformly if the Reynolds number is sufficiently low. We shall concentrate on this case for our discussion of threads, since recently a new type of similarity solution has been found that describes the thinning of jets at very high viscosities (Papageorgiou, 1995). If the Reynolds number is small initially, the evolution is governed by the Stokes equation (16). Going through the same thin filament analysis as was developed for the case of the Navier-Stokes equation, one effectively drops the inertial terms $\partial_{t} v+v v^{\prime}$ in the lubrication equation for the axial velocity (79). Since surface tension and viscous forces are now the only forces to be balanced, there is additional scaling freedom in the equations. As a result, singular solutions can have the form

$$
\begin{aligned}
& h_{s}\left(z^{\prime}, t^{\prime}\right)=\left|t^{\prime}\right| \phi_{\mathrm{St}}(\xi), \\
& v_{s}\left(z^{\prime}, t^{\prime}\right)=\left|t^{\prime}\right|^{\beta-1} \psi_{\mathrm{St}}(\xi), \\
& \xi=z^{\prime} /\left|t^{\prime}\right|^{\beta},
\end{aligned}
$$

where the exponent $\beta$ is to be determined. The scaling (111) is recovered for $\beta=1 / 2$. Although Eq. (144) suggests the existence of a solution for any $\beta$, a particular $\beta$ is selected by the dynamics, as Papageorgiou showed. The value is close to $\beta=0.175$, which means that the axial scale of this solution contracts at a much slower rate than it does for the asymptotic solution (111). Plugging Eq. (144) into the full equations (79), one finds that the surface tension and the viscous terms scale like $\left|t^{\prime}\right|^{-1-\beta} \approx\left|t^{\prime}\right|^{-1.18}$, while the inertial terms are propor-

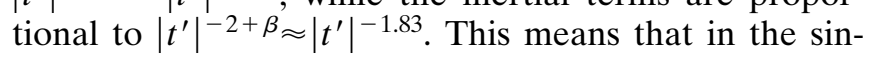
gular limit the terms neglected in the Stokes approximation become larger than those retained. Hence, for $t^{\prime} \rightarrow 0$ the Stokes solution is no longer consistent but must give way to the asymptotic solution, in which all forces are balanced. Note that Papageorgiou (1995) makes an erroneous statement to the contrary.

To derive the Stokes solution, we shall use a presentation somewhat different from Papageorgiou's, using the Lagrangian description of Sec. V.D. This allows one to do almost all calculations analytically. Dropping the inertial term $z_{t t}$ from Eq. (96), one finds

$$
0=\frac{\gamma}{\rho}\left(z_{\alpha}^{-1 / 2}\right)_{\alpha}+3 \nu\left(\frac{z_{t \alpha}}{z_{\alpha}^{2}}\right)_{\alpha},
$$

which can be integrated to give

$$
C(t)=\frac{\gamma}{\rho} H-6 \nu H_{t} H,
$$

where $H(\alpha, t)=z_{\alpha}^{-1 / 2}(\alpha, t)$ is the height of the interface in Lagrangian coordinates and $C(t)$ is independent of $\alpha$. This description was first given by Renardy (1994).
What is remarkable about Eq. (146) is that all spatial derivatives have dropped out. Hence spatial information, for example the characteristic width of the modulation, can only enter through the initial conditions.

We now look for the singular contribution to $H$, relative to the singular point $\alpha_{0}, t_{0}$, in units of $\ell_{\nu}, t_{\nu}$ :

$$
\begin{aligned}
& H(\alpha, t)=\ell_{\nu} H_{s}\left(\alpha^{\prime}, t^{\prime}\right), \\
& \alpha^{\prime}=\left(\alpha-\alpha_{0}\right) / \ell_{\nu}^{3}, \\
& t^{\prime}=\left(t_{0}-t\right) / t_{\nu} .
\end{aligned}
$$

Then the self-similar ansatz equivalent to Eq. (144) is

$$
\begin{aligned}
& H_{s}\left(\alpha^{\prime}, t^{\prime}\right)=\left|t^{\prime}\right| \chi(\zeta), \\
& \zeta=\alpha^{\prime} /\left|t^{\prime}\right|^{2+\beta} .
\end{aligned}
$$

Plugging this into the equations of motion (146), one finds the similarity equation

$$
\bar{C}=\chi+6 \chi^{2}-6(2+\beta) \zeta \chi^{\prime} \chi,
$$

where $\bar{C}$ is a constant. Remembering that $H^{-2}(\alpha, t)=z_{\alpha}(\alpha, t)$, and therefore $\chi^{-2}=F_{\zeta}$ for a suitable $F$, one finds that

$$
\bar{C}=\frac{\int_{-\infty}^{\infty} \chi^{-3}(\zeta) d \zeta}{\int_{-\infty}^{\infty} \chi^{-4}(\zeta) d \zeta} .
$$

Thus the additional constraint (150) is a direct consequence of volume conservation, as expressed by Eq. (93). Equations (149) and (150) can be used to compute the constants $\bar{C}$ and $\beta$. First, Eq. (149) can be integrated to give

$$
\ln \left|\frac{\zeta}{\zeta_{0}}\right|=6(2+\beta) \int_{\chi_{0}}^{\chi} \frac{\zeta^{\prime} d \zeta^{\prime}}{6 \zeta^{\prime 2}+\zeta^{\prime}-\bar{C}} .
$$

From the symmetry of the similarity equations one concludes that $\chi(\zeta)$ is symmetric and takes its minimum at $\zeta_{0}=0: \chi_{0}=\chi(0)$. The expansion of $\chi$ around the minimum looks like

$$
\chi(\zeta)=\chi_{0}+\zeta^{2}+\chi_{4} \zeta^{4}+\cdots
$$

where the quadratic coefficient was normalized to one. By demanding that the singularities in Eq. (151) cancel as $\zeta_{0} \rightarrow 0$, one finds that

$$
\begin{aligned}
& \chi_{0}=\frac{1}{12(1+\beta)}, \\
& \bar{C}=\frac{3+2 \beta}{24(1+\beta)^{2}} .
\end{aligned}
$$

When one does the integral in Eq. (151), the explicit expression for the inverse of $\chi$ is

$$
\begin{aligned}
\zeta= & \left(\frac{6(1+\beta)}{2+\beta}\right)^{(3+2 \beta) / 2}\left[\chi+\frac{3+2 \beta}{12(1+\beta)}\right]^{(3+2 \beta) / 2} \\
& \times\left(\chi-\chi_{0}\right)^{1 / 2} .
\end{aligned}
$$

Now Eq. (150) can be used to derive an eigenvalue relation for $\beta$, using the transformation $d \zeta=\chi^{\prime-1} d \chi$ : 

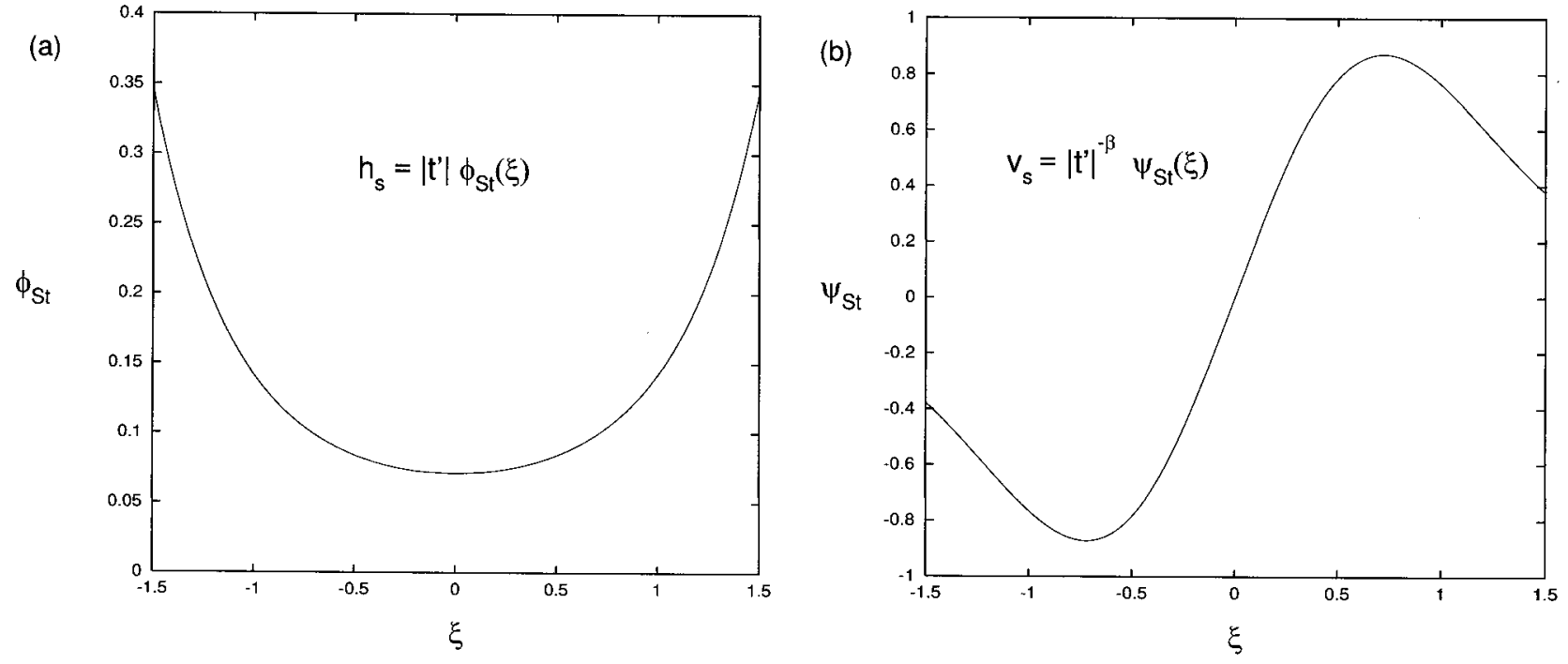

FIG. 28. Scaling functions of the Stokes similarity solution, $\phi_{\mathrm{St}}$ and $\psi_{\mathrm{St}}$.

$$
\frac{3+2 \beta}{24(1+\beta)^{2}}=\frac{\int_{\chi_{0}}^{\infty}\left(d \chi^{\prime} \chi^{\prime} \chi^{3}\right)}{\int_{\chi_{0}}^{\infty}\left(d \chi^{\prime} \chi^{\prime} \chi^{4}\right)} .
$$

Employing both Eqs. (149) and (155), the integrals lead to hypergeometric functions $F$, so one finally has (Gradshteyn and Ryzhik, 1980)

$\frac{(1-\beta)(3+2 \beta)}{(1+\beta)(3-2 \beta)}=\frac{F(-1 / 2-\beta, 1-\beta, 3 / 2-\beta,-3-2 \beta)}{F(-1 / 2-\beta, 2-\beta, 5 / 2-\beta,-3-2 \beta)}$,

which is the desired equation for $\beta$. Incidentally, the particular ratio of hypergeometric functions appearing in Eq. (156) can be evaluated very efficiently in a continued-fraction representation (Bender and Orszag, 1978, p. 396), giving

$$
\beta=0.17487 \ldots \text {, }
$$

in agreement with Papageorgiou's result. Brenner, Lister, and Stone (1996) have shown that the solution thus found is not the only one, but again there is an infinite sequence of solutions. They correspond to the first nonvanishing term in the expansion of $\chi$ being not of second order, as in Eq. (152), but of higher-order. Again, the higher order solutions are much more unstable than the one corresponding to Eq. (157). But this is not all. By a combination of asymptotic analysis and numerics, Renardy (1995) has shown that singularities with power laws different from (144) may occur, depending on initial conditions. In particular, the exponent with which the local radius goes to zero as a function of $\left|t^{\prime}\right|$ may vary continuously between $1 / 2$ and the value 1 of Eq. (144). In the case of slowly varying initial data, however, the present Stokes solution is expected to occur.

Finally, to transform back from Lagrangian coordinates to real space, one has to integrate the relation

$$
z^{\prime}=\int_{0}^{\alpha} H^{-2}(\bar{\alpha}, t) d \bar{\alpha} .
$$

In similarity variables, this amounts to

$$
\frac{\xi}{\bar{\xi}}=\int_{1}^{\phi_{\mathrm{St}}(\xi) / \chi_{0}} \frac{(x+3+2 \beta)^{(1+2 \beta) / 2}}{x(x-1)^{1 / 2}} d x,
$$

where the integral can be done numerically without great fuss. The normalization length $\bar{\xi}$ is arbitrary and depends on initial conditions. For practical purposes one will have to fit it to experiment. Both scaling functions $\phi_{\mathrm{St}}$ and $\psi_{\mathrm{St}}$ in the Stokes case are plotted in Fig. 28. The scaling function $\psi_{\mathrm{St}}$ of the velocity is found from

$$
\psi_{\mathrm{St}}(\xi)=\int_{0}^{\xi} \frac{6 \chi_{0}^{2}+\chi_{0}-\phi_{\mathrm{St}}(\bar{\xi})}{3 \phi_{\mathrm{St}}^{2}(\bar{\xi})} d \bar{\xi} .
$$

A straightforward analysis of the asymptotics for large $|\xi|$ reveals that

$$
\begin{aligned}
& \phi_{\mathrm{St}}(\xi) \sim|\xi|^{1 / \beta}, \\
& \psi_{\mathrm{St}}(\xi) \sim|\xi|^{(\beta-1) / \beta},
\end{aligned}
$$

as is required for a proper matching onto a static outer solution.

Figure 29 shows the middle portion of the neck between two drops in a jet experiment at high viscosity, $\mathrm{Re}=0.32$ (Kowalewski, 1996). As required by the similarity solution of the Stokes equation, the solution is symmetric, with the singular point in the middle. In Fig. 29(a) we have fitted a similarity solution (144) with $\left|t^{\prime}\right|=0.22$ to the neck by adjusting the axial scale. The minimum thickness of the neck, by contrast, is predicted without adjustable parameters and agrees well with experiment. This thickness has more than twice the value one would have expected from the asymptotic similarity solution, which includes inertia. Part (b) of Fig. 29 shows the same neck at a later stage, $\left|t^{\prime}\right|=0.12$, compared with 

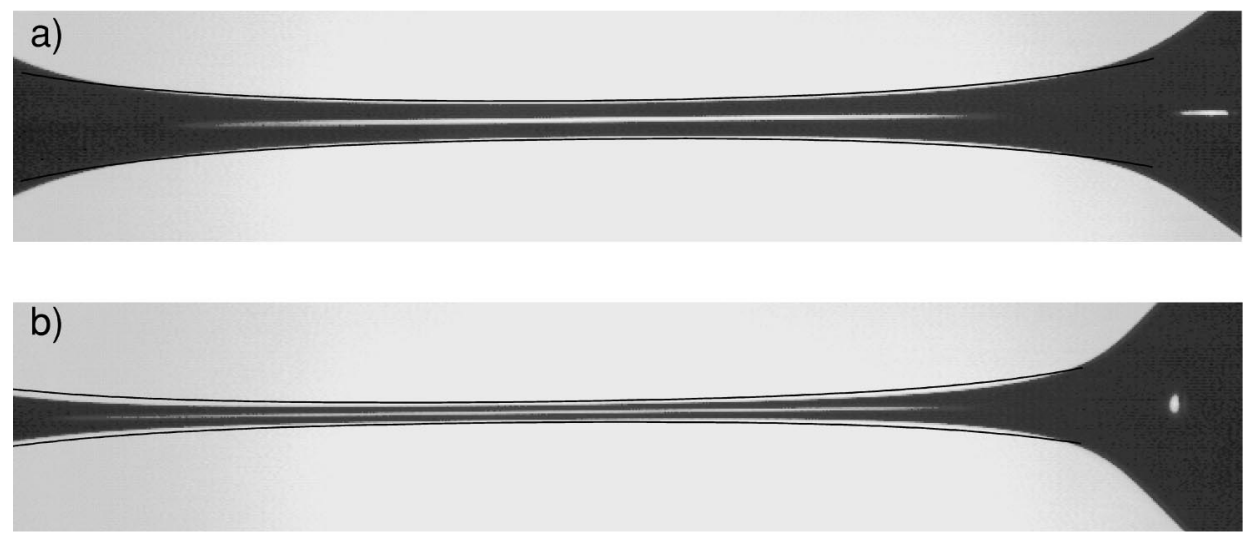

FIG. 29. Two pictures of the thread in between drops for a highly viscous fluid, $\ell_{\nu}=2 \mathrm{~mm}$ [series GLY3 of Kowalewski, 1996], which is also the width of the frame. In (a), $2.8 \mathrm{~ms}$ away from the singularity, a Stokes similarity solution, represented by the solid line, was fitted by adjusting the axial scale. Part (b), $1.5 \mathrm{~ms}$ away from the singularity, contains no adjustable parameter.

the same similarity solution, so here there is no adjustable parameter. There is some mismatch in the thickness, indicating that the Stokes approximation ceases to be valid, in spite of the low initial Reynolds number. Since the exponent $\beta$ is quite small, the length of the neck has hardly changed, although its thickness has halved.

Eventually, however, the symmetric similarity solution must give way to a highly asymmetric asymptotic solution. When this happens depends very much on the viscosity as well as on the initial condition. The transition to the asymptotic solution has been described by Kowalewski (1996) as the formation of a "microthread," while he called the original neck between the drops a "macro-thread." A typical example is shown in Fig. 30. Note that near the drop the macro-thread shrinks at a much faster rate, as required by the diminished thickness of the asymptotic solution.
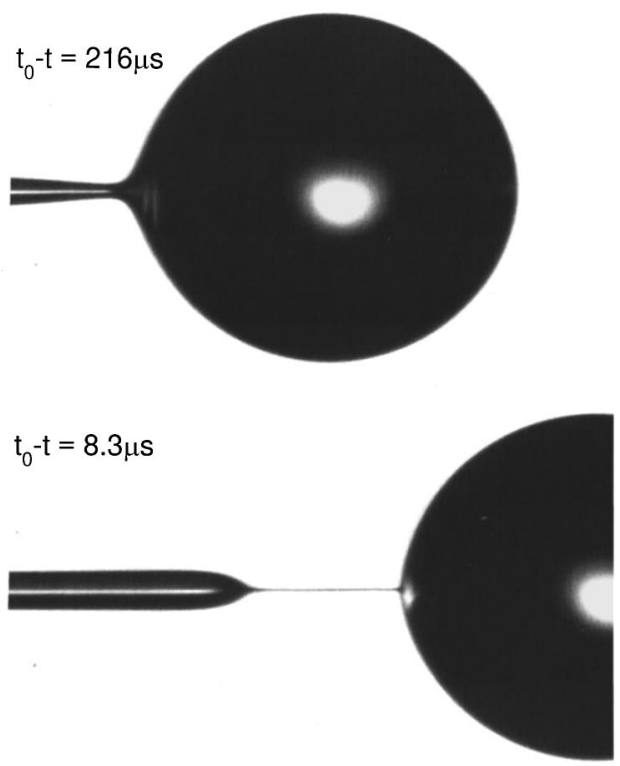

The newly formed micro-thread is highly asymmetric and grows at the expense of the original thread. It is to one of these micro-threads that the asymptotic solution was fitted in Fig. 18. The micro-thread gets longer with increasing viscosity, and in Fig. 18 eventually takes up the entire field of vision. A similar formation of a microthread can also be seen in the case of a falling drop in Fig. 20(a).

\section{B. Low viscosity-cones}

The limit complementary to that of the previous section is that of very large Reynolds number $\operatorname{Re}=\left(L / \ell_{\nu}\right)^{1 / 2}$. The simplest guess is that in this case viscosity is negligible and the pinching process is described by inviscid dynamics, as long as the minimum radius remains much larger than $\ell_{\nu}$. It was for the sake

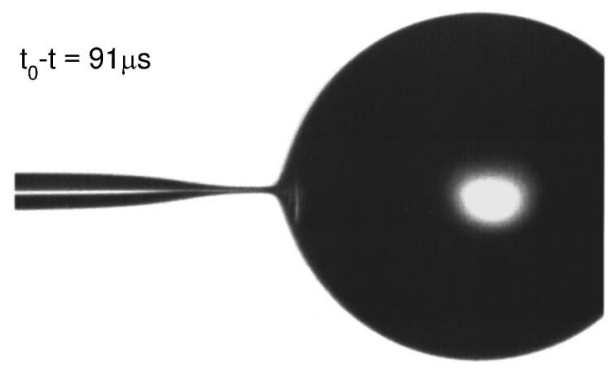

at breakup

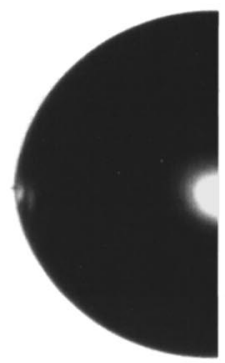

FIG. 30. A sequence of pictures showing the development of a micro-thread out of a macro-thread. The micro-thread represents an asymptotic similarity solution, which has a smaller radius than the Stokes solution at a given time away from breakup (from series MIXD of Kowalewski, 1996). 
of this argument, in fact, that Peregrine et al. (1990) first introduced the scale $\ell_{\nu}$. Hence one eliminates the viscous contribution in Eq. (79), ending up with an equation first studied in detail by Ting and Keller (1990). Analogous to the low-Reynolds-number limit of the previous subsection, this amounts to an additional scaling freedom of possible pinching solutions, which now have the general form

$$
\begin{aligned}
& h_{s}\left(z^{\prime}, t^{\prime}\right)=\left|t^{\prime}\right|^{2-2 \alpha} \phi_{\mathrm{Eu}}(\xi), \\
& v_{s}\left(z^{\prime}, t^{\prime}\right)=\left|t^{\prime}\right|^{\alpha-1} \psi_{\mathrm{Eu}}(\xi), \\
& \xi=z^{\prime} /\left|t^{\prime}\right|^{\alpha} .
\end{aligned}
$$

Since on the scales considered here $\ell_{\nu}$ and $t_{\nu}$ are assumed to be effectively zero, the distance from the singularity $z^{\prime}, t^{\prime}$ must be measured in units of the outer scale $L$ and the corresponding time $T=\left(\rho L^{3} / \gamma\right)^{1 / 2}$. Hence the radial and axial length scales of the solution (161) are

$$
\begin{aligned}
& \ell_{r}=L\left(\left(t_{0}-t\right) / T\right)^{2-2 \alpha}, \\
& \ell_{z}=L\left(\left(t_{0}-t\right) / T\right)^{\alpha} .
\end{aligned}
$$

For the rest of this subsection, we shall conveniently nondimensionalize all quantities with $L$ and $T$. As long as $\alpha<3 / 2$, the slenderness assumption $\ell_{r} \ll \ell_{z}$ is valid, and Eq. (161) is an asymptotic solution to the full Navier-Stokes equation (17)-(19) with zero viscosity, usually called the Euler equation. If, moreover, $\alpha<1 / 2$, the approximation of zero viscosity is self-consistent in the sense that the viscous contribution $\nu v^{\prime \prime}$ becomes asymptotically irrelevant as $t^{\prime} \rightarrow 0$. As shown by Ting and Keller, there exist solutions of the similarity equations consistent with proper boundary conditions at infinity for any $\alpha$, each having two free parameters. So, unlike the viscous case, there is no known mechanism that selects a specific solution.

In Sec. V.D we looked at the inviscid lubrication equations and recast them in the form (97). Some exact solutions other than Eq. (161) have been found, but do not correspond to pinching solutions with the local radius going to zero. In fact, the existence of the infinite sequence of conservation laws (106) and (107) poses a subtle problem: Pinching solutions require that $\alpha<1$, which also implies that the velocity goes to infinity at the pinch point. Thus, regardless of how fast the axial extension of the pinch region shrinks, conserved quantities $Q_{i}^{ \pm}$of sufficiently high order, as given by the densities (106) and (107), will diverge. This means the pinching solutions (161) are inconsistent with finite initial conditions unless higher-order terms are taken into account in the equations which might destroy some of the conservation laws.

Of particular importance among the exponents $\alpha$ is the case $\alpha=3 / 2$, which also marks the borderline of the validity of the slenderness assumption. This value is special because the dependence on the outer scale $L$ drops out in Eq. (162) and both radial and axial scales are given by

$$
\ell_{r}=\ell_{z}=\ell_{\mathrm{KM}}=t^{\prime 2 / 3} \text {. }
$$

Thus, in the absence of any viscous length scale, $\alpha=2 / 3$ is the only exponent that leads to a solution independent of external scales, like the nozzle radius $r_{0}$. This was first noticed by Keller and Miksis (1983), who also observed that for this scaling similarity solutions of the full inviscid equations exist, without resorting to any slenderness assumption. Using this, they computed similarity solutions of the equations (9)-(11) for inviscid, irrotational flow in the case of a receding wedge after breakup. Later Peregrine et al. (1990) applied this idea to the axisymmetric breakup problem before and after the singularity. They noticed that the cone-shaped surface profiles observed in low-viscosity fluids are in qualitative agreement with a scaling law, Eq. (163), which predicts $h$ to have a linear behavior away from the singularity. However, no quantitative test of these ideas was performed. Subsequently, Eggers and Dupont (1994) questioned the validity of the scaling law (163) on the basis of their simulations of the viscous lubrication equations (79) and (80) with the full pressure term (82).

First, Eggers and Dupont (1994) noted that this simple approximation performs remarkably well in reproducing the experiments of Peregrine et al. before breakup. But using detailed simulations of the lubrication equations, Eggers and Dupont then noted severe departures from the scaling law (163), even when $h_{\text {min }} \gg \ell_{\nu}$. The reason is a very sharp increase in the gradients of $h$ and $v$ before $h_{\text {min }}$ goes to zero, which they interpreted as a singularity of the inviscid equations, which is stopped only by viscosity. This singularity makes the viscosity become important while $h_{\min } \gg \ell_{\nu}$, and thus contradicts the assumptions implicit in Eqs. (161) and (163). In particular, this implies that the inviscid equations become inconsistent before $h_{\min }$ goes to zero and therefore are not able to describe pinching. The analysis of the behavior at small viscosities has recently been refined by Brenner et al. (1997) and augmented by quantitative experiments. Experiment and simulation at smaller viscosities than those studied by Eggers and Dupont show the scaling (163) of the minimum height, but only over a small range of scales.

The experiments of Brenner et al. consist of a highspeed motion picture of a drop of water falling from a 7 -mm pipette. The time between frames is $23 \mu \mathrm{s}$. The measured values of $h_{\min }$ are shown in Fig. 31. The data are consistent with the $2 / 3$ scaling (163) over $11 / 2$ decades in time, but a deviation begins to show at the times closest to rupture. In Fig. 32 the full surface profiles have been collapsed according to Eq. (161) with $\alpha=2 / 3$, thus

$$
h_{s}\left(z^{\prime}, t^{\prime}\right)=\left|t^{\prime}\right|^{2 / 3} \phi_{\mathrm{Eu}}\left(z^{\prime} /\left|t^{\prime}\right|^{2 / 3}\right) .
$$

The minimum of $\phi_{\mathrm{Eu}}$, as well as the slope to its right, remains constant, thus showing reasonable agreement with Eq. (164). However, on the steep side to the left of the minimum the slope of the profile increases sharply, while Eq. (164) would predict it to remain constant.

These findings are corroborated by the numerical simulations Brenner et al. performed for the same parameters, using the lubrication equations. However, an 


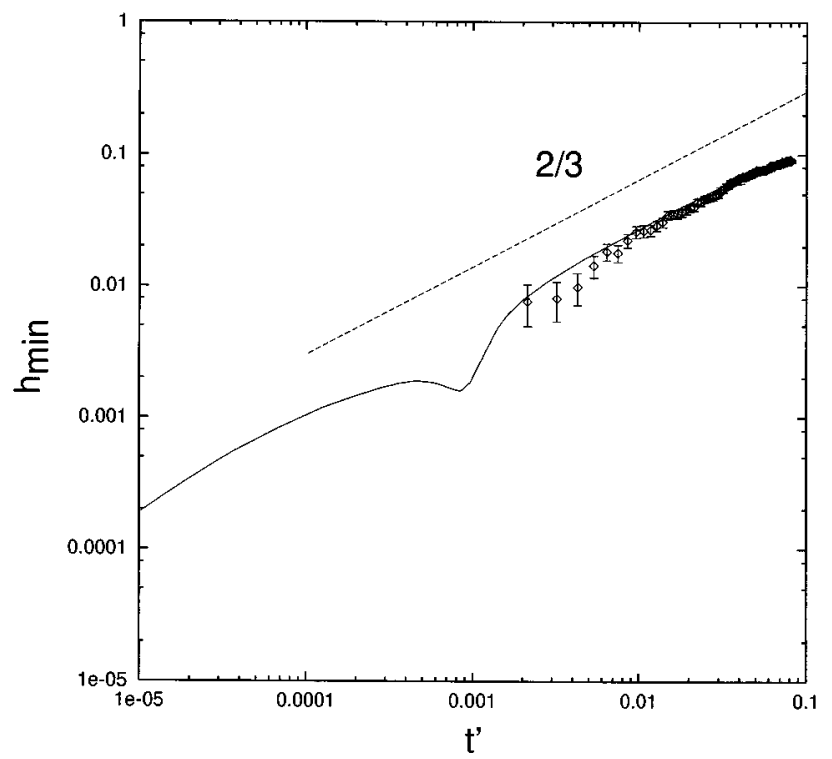

FIG. 31. Experimental measurements (shown as diamonds) of the minimum radius $h_{\min }$ of a neck of water in units of the nozzle radius versus the time distance $t^{\prime}$ from breakup (Brenner et al., 1997). A least-squares fit to the data gives the fit $h_{\text {min }} \sim t^{\prime 0.68}$, dimensional scaling is shown as the dotted line for comparison. The solid line shows the result of a numerical simulation, described below.

even more drastic departure from Eq. (164) occurs below the experimentally resolved scales, corresponding to a breakdown of dimensional scaling long before the scale $\ell_{\nu}$ is reached. Figure 33 shows the minimum radius from numerical simulations, whose resolution permits a followup on the evolution of $h_{\min }$ to scales much smaller than in experiments. Note that in units of the nozzle radius, the viscous length $\ell_{\nu}$ is $1.2 \times 10^{-7}$-still more

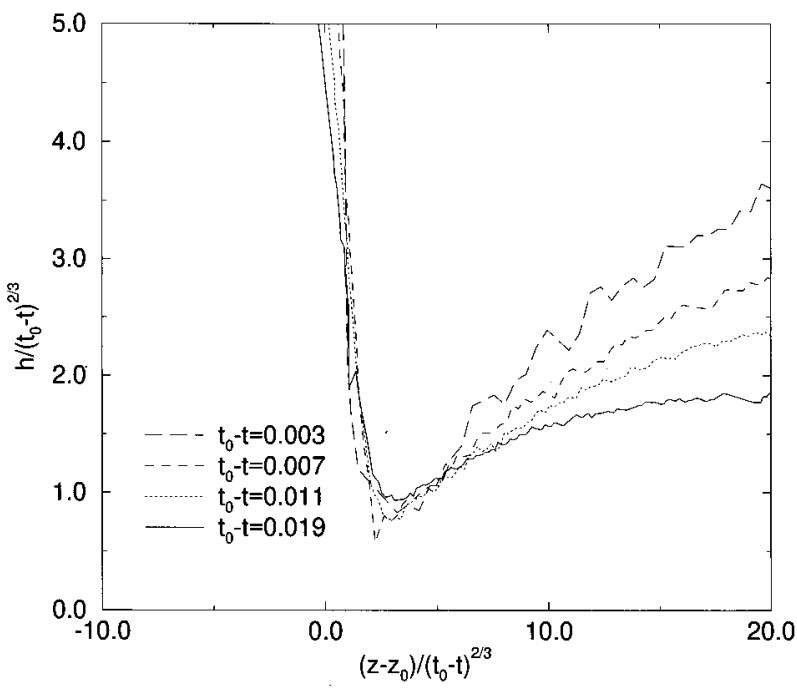

FIG. 32. The scaling function $\phi_{\mathrm{Eu}}$ as calculated from Eq. (164) using the same experimental data as in Fig. 31. The slope to the left of the minimum increases sharply in the time interval shown.
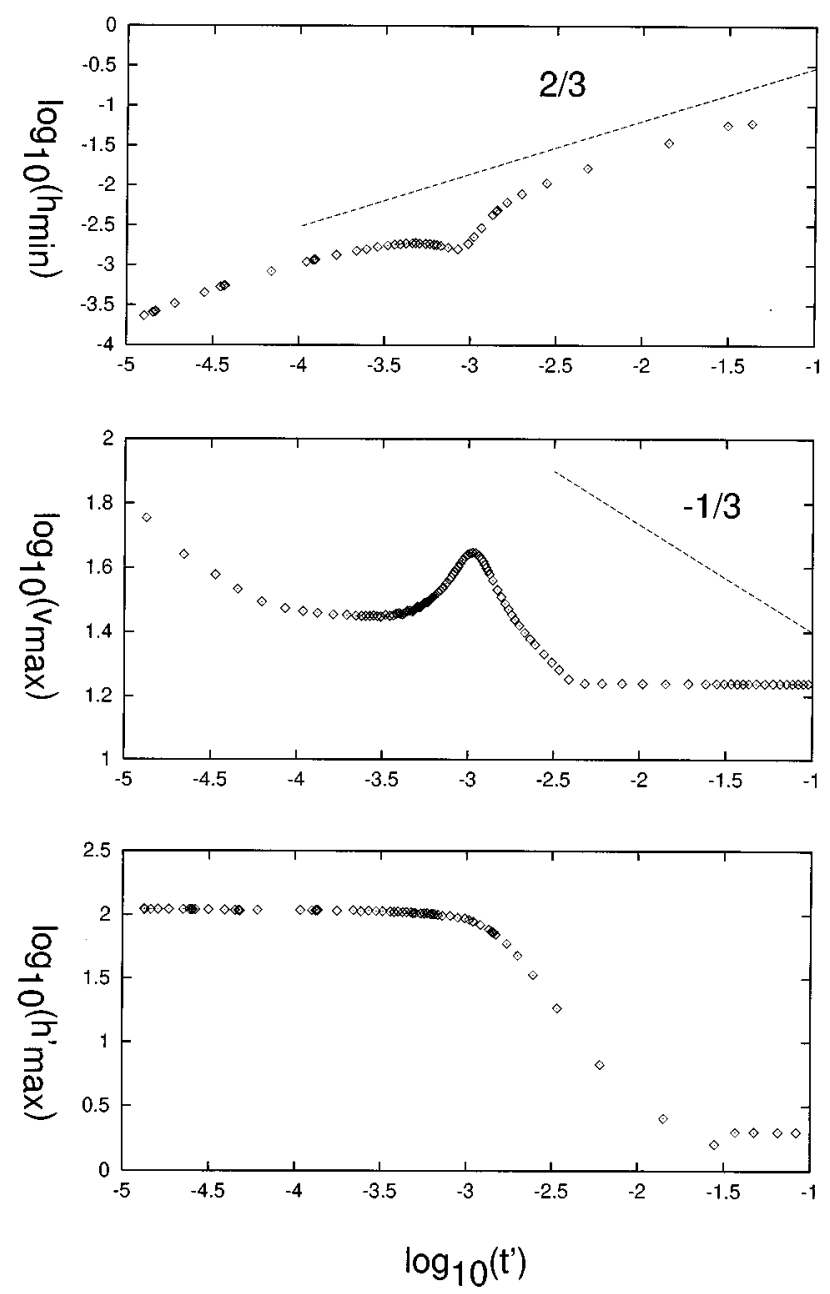

FIG. 33. Numerical simulation of a dripping faucet, for the same parameter values as in experiment (see Fig. 31). Shown are the minimum radius, the maximum velocity, and the slope at corresponding times. At $t^{\prime} \approx 10^{-3}$ a sharp dip occurs, which corresponds to the slope's saturating at its maximum value. The dotted lines are the predictions of the inviscid theory.

than three orders of magnitude below the smallest $h_{\text {min }}$. For times down to the experimentally resolved ones, the $2 / 3$ law is confirmed, in agreement with experiment; see Fig. 31.

On scales slightly smaller, a drastic departure occurs and $h_{\min }$ even increases, forming a dip in the time dependence of $h_{\min }$. This increase was not seen in the earlier simulations at higher viscosities, where it was smoothed out by viscosity. We also show in Fig. 33 the maximum velocity, which should scale like $t^{\prime-1 / 3}$ according to the inviscid scaling law. Clearly, the observed scaling of the velocity is not even close to this prediction. The departure from $2 / 3$ scaling in the minimum height coincides with the slope's reaching its maximum value at $t^{\prime} \approx 10^{-3}$, as can also be seen in Fig. 33. Before that time, starting from $t^{\prime} \approx 10^{-2}$, a rapid increase occurs. The same increase is observed again in the rescaled profiles of Fig. 34, which correspond to the experimental pictures of Fig. 32. This suggests that the inviscid equations have a singularity at finite time $t_{0}-t$ away from breakup, 


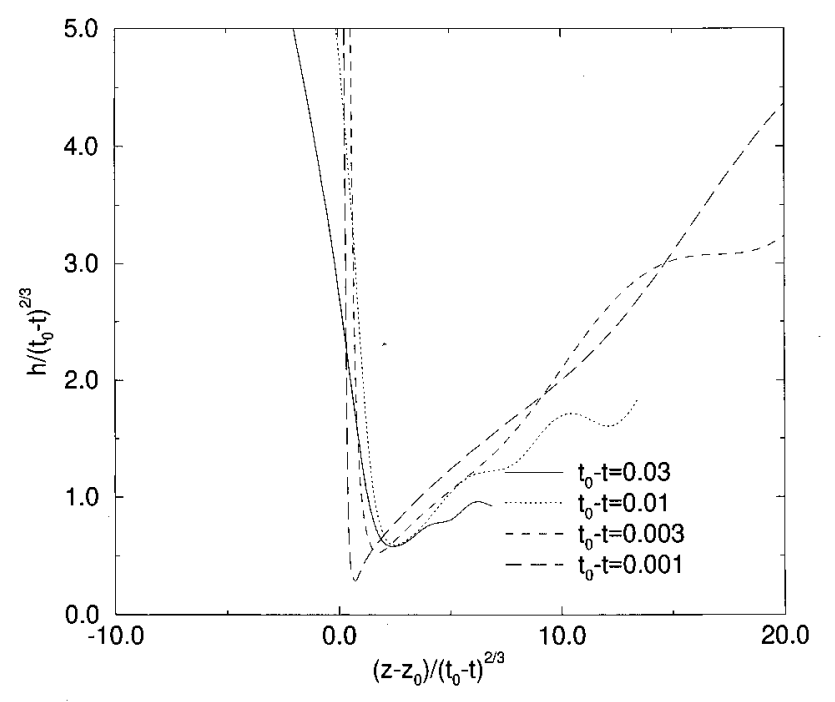

FIG. 34. Rescaled profiles $\phi_{\mathrm{Eu}}$ from numerical simulations, corresponding to the experimental data of Fig. 32. The longdashed line is for a time closer to breakup than shown experimentally.

at which the maximum slope goes to infinity.

To test for the existence of this singularity in more detail, a series of simulations with decreasing viscosity, but for the same initial conditions, was performed by Brenner et al. (1997). The result of these simulations for the liquid bridge geometry without gravity is shown in Fig. 35, which gives the maximum slope as a function of the time distance from the pinching singularity. The change in viscosity between the lowest and the highest Reynolds number is four orders of magnitude. While the initial evolution is almost unaffected by viscosity, the maximum slope increases drastically with Reynolds number. The time at which the maximum slope is

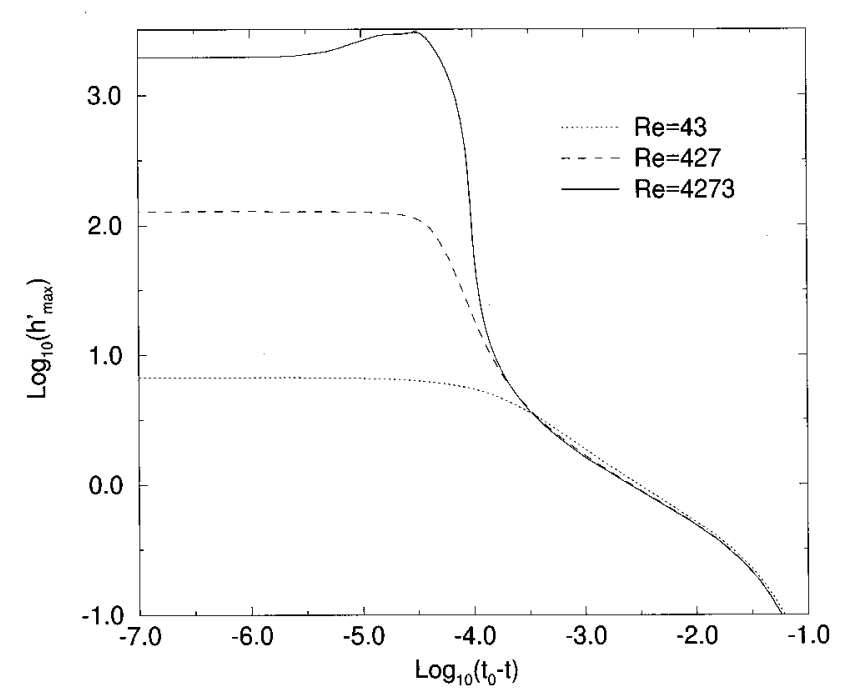

FIG. 35. Maximum slope as a function of time for various Reynolds numbers in a simulation of the liquid-bridge geometry. A singularity of the inviscid equations can be estimated to occur at $t^{\prime} \approx 10^{-4}$. reached remains almost constant, since the rise gets sharper. Extrapolating to zero viscosity one confirms that the inviscid equations have a singularity at a finite time away from breakup. As the viscosity is reduced, the range of validity of the inviscid equations does not expand. Thus, at least within the framework of the lubrication equations, the Keller-Miksis theory can at best be valid over a small range of scales.

Recently, the structure of the inviscid singularity of the lubrication equations with full curvature term has been cleared up in more detail (Eggers, 1997). The solution

$$
\begin{aligned}
& h(\bar{z}, \bar{t})=H+\bar{t}^{-1 / 2} f\left(\frac{\bar{z}+V_{s} \bar{t}}{\bar{t}^{-3 / 2}}\right), \\
& v(\bar{z}, \bar{t})=V+\bar{t}^{-1 / 2} g\left(\frac{\bar{z}+V_{s} \bar{t}}{\bar{t}^{3 / 2}}\right)
\end{aligned}
$$

explicitly incorporates the fact that both the height and the velocity remain finite at the singularity. The spatial and the temporal distances from this singularity, termed "curvature" singularity in Brenner et al. (1997), are measured by

$$
\bar{z}=z-z_{c}, \quad \bar{t}=t_{c}-t .
$$

Since the power in the denominator of

$$
\eta=\frac{\bar{z}+V_{s} \bar{t}}{\bar{t}^{-3 / 2}}
$$

is greater than the power $t^{1 / 2}$ in front of $f$ and $g$, the slope of $h$ and $v$ goes to infinity asymptotically and the pressure is dominated by the highest derivatives in Eq. (82).

When we analyze the similarity equations corresponding to Eq. (165), an analytical solution for the derivative $f^{\prime}$ of the similarity function of the height can be found, which has the form

$$
f^{\prime}(\eta)=\left(\frac{2\left(V_{s}-V\right)^{2}}{H}\right)^{1 / 3} \phi_{0} F\left(\phi_{0}^{3 / 2}\left(\eta-\eta_{0}\right)\right) .
$$

Here $\phi_{0}$ and $\eta_{0}$ are free constants and $F$ is given implicitly by

$$
\xi=\frac{1}{8 F^{3 / 2}}(1+2 F)(1-F)^{1 / 2} .
$$

The maximum of the function $F$, which is shown in Fig. 36 as the solid line, has been chosen to be at $\eta=0$ with the half-width normalized to one. In the same figure, the analytical result (169) is compared with a simulation at very high Reynolds number, which ensures that viscosity is not yet important.

After the curvature singularity is regularized by viscosity, the structure of the solution is more complicated: there must be a thin layer around the position of maximum slope where viscosity is important. In other parts of the solution, where the profile remains relatively flat, the dynamics will be governed by inviscid equations. A more detailed characterization of this boundary-layer structure or its possible scaling properties remains an 


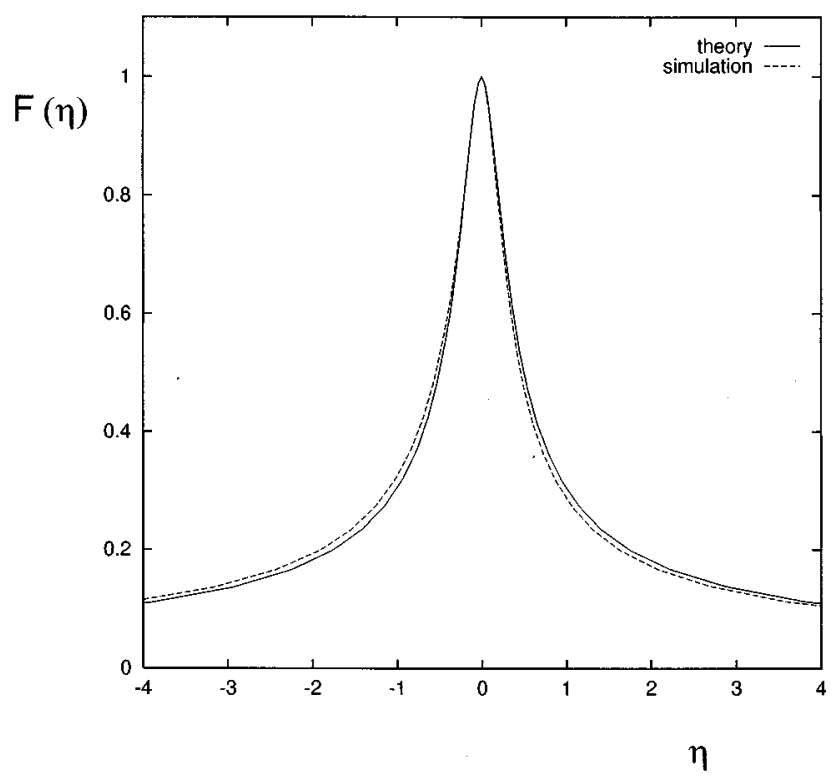

FIG. 36. The similarity function (169) for the inviscid singularity of the lubrication equations. Theory is compared with a numerical simulation at $\operatorname{Re}=5 \times 10^{9}$, at a time when the maximum slope has reached $10^{4}$.

open problem. Likewise, the reason for the appearance of the exponent $\alpha=2 / 3$, which seems to be selected over some range of scales, has not been understood on a deeper level.

\section{Satellite drops}

The understanding of satellite drops and their possible control has been the driving force behind much of the research on drop formation. It seems they were first observed by Plateau (1849) in his experiments with an unstable cylinder of viscous fluid suspended in another (see Fig. 37).

In air, where drops are not held in place by the outer fluid, they are much harder to observe, but still did not escape the attentive eyes of Guthrie (1864). He noticed tiny drops being thrown upward towards the nozzle in a dripping-faucet experiment and conjectured that they resulted from the profile's not being spherical at the point of breakup. Rayleigh (1882), using stroboscopic methods, saw small drops appearing between main drops in jet experiments and called them "spherules." For engineering purposes, jets are of particular importance, the ink-jet printer playing the role of a prototypical application (Pimbley and Lee, 1977; Chaudhary and Maxworthy, 1980b). As the drops separate from the ink jet, they are individually charged and deflected onto their target by an electric field. The smaller satellites are deflected differently, producing an undesirable "halo" around the intended point. So the goal is to avoid satellite formation altogether or to avoid having the satellites charged.

Under normal circumstances, satellite drops are a universal feature of breakup, quite independent of the ex-
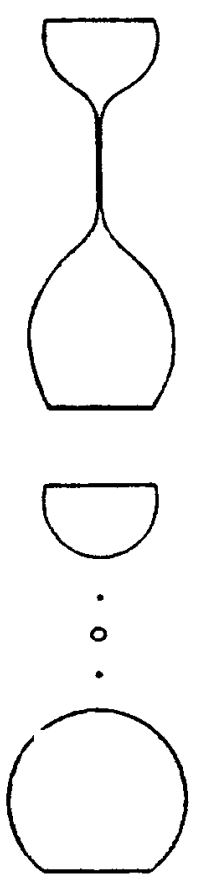

FIG. 37. The final stages of the breakup of a column of olive oil in a mixture of alcohol and water, as observed by Plateau (1849).

perimental configuration. Qualitatively, they come about through two properties of the pinching singularity:

(i) The singularity is localized, producing pinchoff at a point. Since only a small amount of fluid is involved, it acts on time scales much shorter than the growth of disturbances on the jet, once it sets in.

(ii) The singularity is asymmetrical, so the only way it can be matched onto an outer solution is by pinching next to a drop, the steep part forming on the side of the drop, the flat part merging into the flat neck.

Hence the neck is pinched off from either side and eventually contracts into a satellite drop. By looking back at the sequence of pictures in Fig. 6, one can see this very clearly. Although the neck is initially thinnest in its middle section, localized pinching sets in only at the time of the last picture of the first row, after a wellseparated drop has begun to form. From this point to breakup in the next picture the outer part of the solution has hardly moved, illustrating the separation of time scales. Before the neck can retract, it pinches on its upper end, and thus a satellite is formed. The same arguments of course apply to jet decay, so within one period two drops are produced, a main drop with volume $V_{d}$ and a satellite drop with a much smaller volume $V_{s}$. At long wavelengths the neck becomes very long and is subject to secondary breakings, which we shall disregard here. We are going to focus on flow at low viscosities, but similar mechanisms are present at higher viscosities, as can be seen in the pictures of "micro-threading" (Fig. 30).

The total volume of both main drop and satellite is controlled by mass conservation. That is $V_{d}+V_{s}$ is equal to the volume of fluid leaving the orifice during one period, and thus 


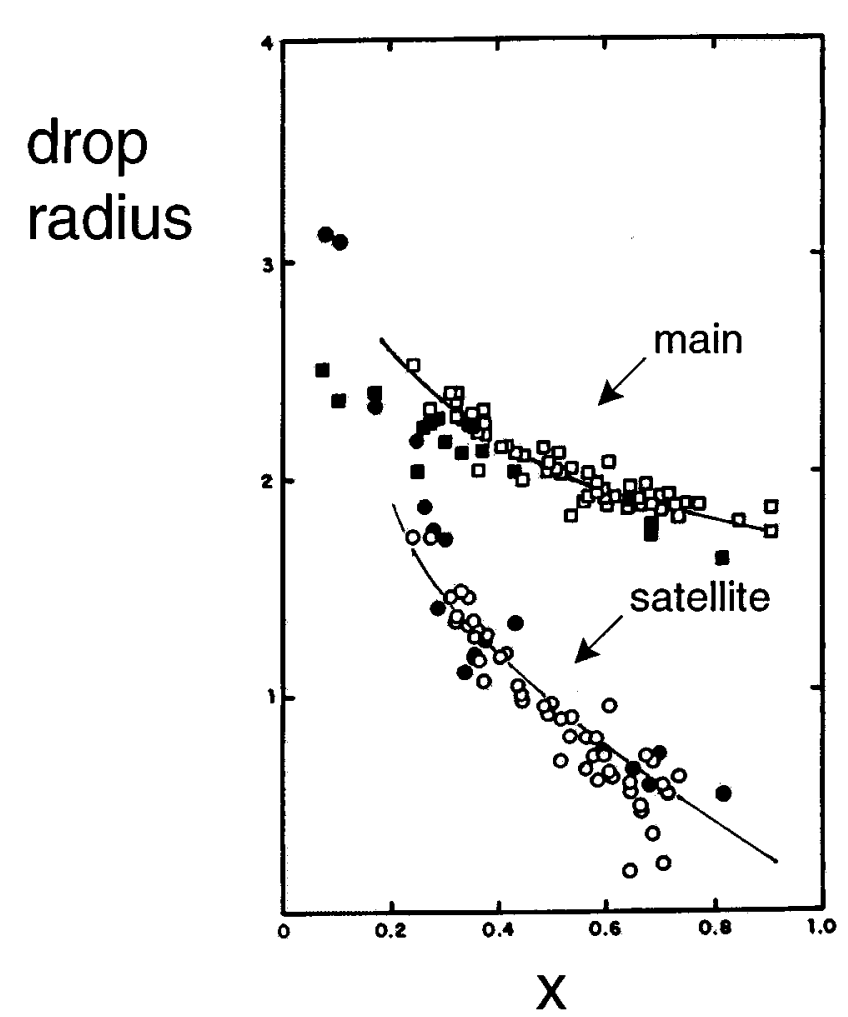

FIG. 38. Radius of main and satellite drop as a function of the reduced wave number. Experimental data by Rutland and Jameson (1970, solid symbols) and Lafrance (1975, open symbols). The lines represent the radius of the main and the satellite drop according to the simulations by Ashgriz and Mashayek (1995).

$$
\left(\frac{r_{d}}{r_{0}}\right)^{3}+\left(\frac{r_{s}}{r_{0}}\right)^{3}=\frac{3 \pi}{2 x} .
$$

Here $r_{d}$ and $r_{s}$ are the equilibrium radii of drop and satellite, respectively. Measurements of $r_{d}$ and $r_{s}$ were performed by Rutland and Jameson (1970) and Lafrance (1975). As shown in Fig. 38, the volume of the satellite drop decreases disproportionally as $x$ is increased. Very close to $x=1$ measurements become less controlled as one is approaching the regime of random breakup. For $x \leqq 0.2$, satellite and main drops become similar in size. Drop sizes are very robust, as no significant dependence on the perturbation amplitude or the Weber number has been reported. This statement is amplified by the fact that simulations with idealized boundary conditions and even perturbation theory have been quite successful in predicting the correct drop sizes. The first comparison was carried out by Rutland and Jameson (1970), using Yuen's (1968) theory. The third-order theory was applied up to the point where the local radius first goes to zero-way beyond the range of applicability of the theory. The other breaking point was extrapolated from this profile. Mass conservation [Eq. (170)] was usually valid to within $10 \%$. The fact that this crude theory gave qualitatively correct results indicates that the drop volume is set early in the evolution of the surface. Once some local minimum is produced, pinch- ing sets in before the drop volumes can change appreciably. Thus only the position of the initial indentations are needed. An even better agreement between perturbation theory and experiment was reported by Lafrance (1975). However, as we discussed at length in Sec. IV.Cं, this solution is erroneous and we feel unable to judge the significance of this agreement.

Simulations have been even more successful in predicting drop volumes. Mansour and Lundgren (1990) employed a simulation of inviscid, irrotational flow, while Ashgriz and Mashayek (1995) simulated the Navier-Stokes equation at $\mathrm{Re}=200$. The results of the latter group are included as solid lines in Fig. 38 and can be seen to agree with experiment to within experimental scatter. Two significant simplifications were made in the simulations: First, the perturbations were uniform and did not grow in space. Second, the simulation did not represent the stationary state of a decaying jet, whose downstream side has a free-boundary condition. Both simplifications considerably influenced the profiles, but had little effect on the total volume within a neck. Note also that Eq. (170) was obeyed exactly by the fully nonlinear calculation.

Apart from the volumes $V_{d}$ and $V_{s}$, the most relevant parameters are the velocities $v_{d}$ and $v_{s}$ of drop and satellite. They determine whether the satellite will merge with the drop leading it ("forward merge," $v_{s}>v_{d}$ ) or with the one following it ("rear merge," $v_{s}<v_{d}$ ). In the limiting case $v_{s}=v_{d}$ ("no merge" or "infinity condition"), the satellite will not merge at all, which is particularly undesirable, as satellite drops will persist far downstream. Analogous to Eq. (170), there is an exact relation involving $v_{d}$ and $v_{s}$, which represents momentum conservation. In it, the momentum $\rho\left(v_{d} V_{d}+v_{s} V_{s}\right)$ carried by the drop and the satellite equals the momentum flux of the unperturbed jet, integrated over one period. In addition to the momentum carried by the fluid, there is a contribution coming from surface tension, which pulls the fluid back towards the nozzle. In the case of an unperturbed jet, this is just the excess pressure $-p=-\gamma / r_{0}$, integrated over the cross section of the jet. Here we have assumed that the initial perturbations on the jet are small. This leads to the second exact balance,

$$
v_{j}\left(r_{d}^{3} v_{d}+r_{s}^{3} v_{s}\right)=\frac{3 \pi}{2 x}\left(v_{j}^{2} r_{0}^{3}-\frac{\gamma}{\rho} r_{0}^{2}\right) .
$$

As expected, the main drop will be slowed down relative to the jet velocity. In fact, neglecting the contribution from the satellite drop, one finds from Eqs. (170) and (171) that

$$
v_{d} \approx v_{j}-\frac{\gamma}{\rho} \frac{1}{r_{0} v_{j}} .
$$

This result was also obtained by Schneider et al. (1967), except that they had an erroneous factor of two in front of the second term on the right. If this is corrected, agreement with their measurements of the drop velocity improves substantially. 
(a)
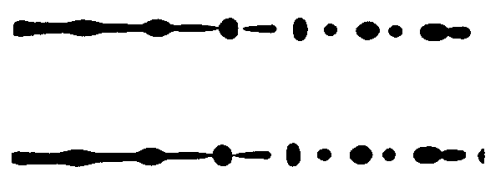

(b)

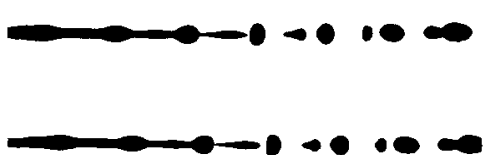

(c)

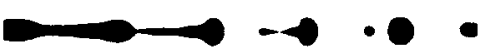

FIG. 39. Photographs of satellite formation at three different driving amplitudes, increasing from (a) to (c), at a reduced wave number $x=0.4312$ (Chaudhary and Maxworthy, 1980b). In (a) the satellite breaks first at its front and then at its rear. In (b) the situation is the same, but the time in between the two events is much shorter. In (c) the satellite breaks first at its rear.

The velocity of the satellite, however, can vary greatly owing to its small mass. Its value will depend on the details of the breakup. The most extensive experimental investigation of satellite formation has been performed by Chaudhary and Maxworthy (1980a,1980b). Figure 39(a)-(c) shows their photographs of jets with varying disturbance amplitudes. At low driving [Fig. 39(a)], the neck breaks first on its front, while at the highest driving [Fig. 39(c)] it breaks on its rear. The latter case means that drop and satellite detach as a single unit, and only later do the two separate. At intermediate driving amplitudes, there is a transition region where the pinching becomes more and more symmetric, causing the breakup in front and behind the neck to occur almost simultaneously, as can be seen in Fig. 39(b). This gradual transition can best be characterized by the time distance $t_{i}$ between the two breakings, which has been called the "satellite interaction time" by Pimbley and Lee (1977). Its significance lies in the fact that it is a measure of the momentum transferred between the two breakings. In case (a), there is a strong backward pull on the satellite, causing it to rear merge, while in (c) it merges with the drop leading it. If $t_{i}$ is very small, as in (b), the momentum transfer is also small and the total momentum of the satellite is given mostly by the velocity distribution at the first breaking. Accordingly, it may either rear merge or forward merge, as in Fig. 39(b), and a prediction of the merging characteristics is difficult.

The focus of Pimbley and Lee's (1977) and Chaudhary and Maxworthy's (1980b) work is on the dependence of the breakup characteristics on the reduced wave number $x$ and on the driving amplitude. Their results agree only qualitatively in that (i) there is a transition from rear merging to forward merging as the driving amplitude is increased, corresponding to a transition

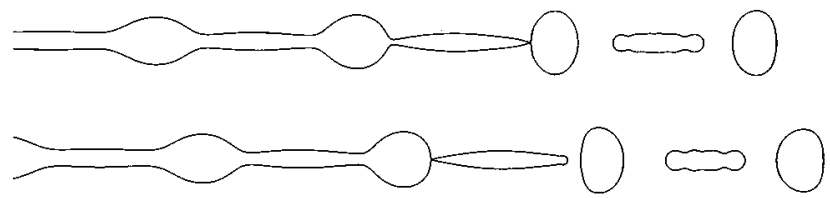

FIG. 40. Simulation of satellite formation in the stationary state of a decaying jet. The dimensionless wave number is $x=0.4312$, the Reynolds number $\mathrm{Re}=39$, and the Weber number $\beta^{2}=239$. The satellite first separates at its front, but almost simultaneously at its rear.

from separation in front of the neck to separation on its rear, and (ii) the critical amplitude that corresponds to the no-merge condition increases with wave number. Left out of their investigation was the Weber number, which has been shown by Vasallo and Ashgriz (1991) to have a significant effect as well.

No accepted explanation exists of the observed transition from front to rear pinching. It was suggested by Pimbley and Lee that the transition is connected to the growth of the second harmonic component or octave above the fundamental input, which they also tried to confirm within a second-order perturbation calculation. Indeed, if a component with a shorter wavelength has had a chance to grow sufficiently with respect to the fundamental, which will be the case at low driving, it will produce an additional growth of surface perturbations across the neck. Thus the neck will break first on its front.

This idea was tested within the one-dimensional model (79), (80), (82) by Eggers (1995c). The jet was broken as described in more detail by Brenner et al. (1997) whenever the radius reached a minimum height of $10^{-3} r_{0}$. After a number of breakings (typically 50100 ), the jet reached a stationary state in which the entire breaking process had the same periodicity $T=1 / f$ as the driving. This was the first time such a stationary state had been established in a numerical simulation. Figure 40 shows stationary jet breakup at a reduced wave number $x=0.4312$ and at weak driving $\epsilon=0.04$, which led to breakup 16 wavelengths away from the nozzle, similar to Fig. 39(a). The Reynolds and Weber numbers were the same as in the experiment performed by Chaudhary and Maxworthy. The neck formed symmetrically, causing it to pinch at both ends at almost the same time. In disagreement with experiment, the simulated breakup remained almost identical as the driving amplitude was increased. This was because the amplitude of harmonics generated by the nonlinear interaction was very small, so it could not affect the breakup.

This situation changes if a small component of a harmonic frequency is added to the driving. The existence of higher harmonic components in the driving by realworld nozzles has been recognized and measured before (Taub, 1976; Chaudhary and Maxworthy, 1980b; Torpey, 1988). They are produced by nonlinearities of the piezocrystal and by the fact that the velocity profile coming out of the nozzle is not constant, but is more or less parabolic, depending on the length of the nozzle. This 
(a)

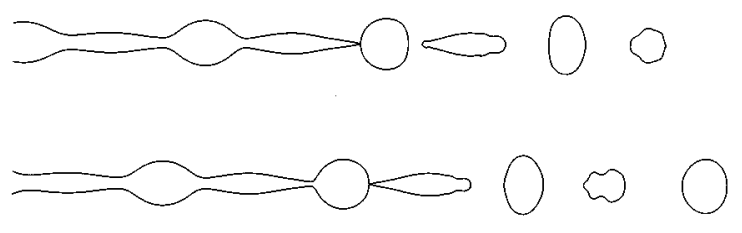

(b)

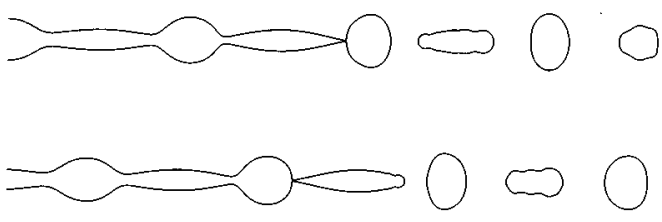

FIG. 41. Same as in Fig. 40, but with the second harmonic component with $1 / 10$ of the amplitude of the fundamental added. Part (a) shows the case of low driving, (b) the case of higher driving. A transition to almost symmetrical pinching is observed.

inevitably translates into nonsinusoidal modulations of the jet. The effect of a nontrivial velocity profile on the jet evolution has been studied directly in an interesting paper by Razumovskiǔ (1993).

The effect of the second harmonic with $1 / 10$ of the amplitude of the fundamental is shown in Fig. 41(a). At a reduced wave number of 0.4312 the growth of the second harmonic is faster than that of the fundamental according to linear theory. Thus at low driving, when there is sufficient time for the harmonic to grow, it may have an appreciable effect. Indeed, the profiles look remarkably similar to the experimental ones of Fig. 39(a), which are also at low driving. There is considerable growth of surface perturbations across the neck, causing it to separate at its front, while there is not much indentation yet on its rear. Note that according to linear theory the amplitude of the second harmonic should still be $1 / 6$ of the fundamental at breakup, so nonlinear effects must also play an important role. If the input is stronger, but the amplitude ratio of the second harmonic to the fundamental is kept constant [see Fig. 41(b)], the harmonic has much less time to grow. Thus the breakup resembles the one without higher harmonic driving (Fig. 40), being much more symmetric [see Fig. 41(b)]. This transition to symmetric pinching with increased driving amplitude is similar to experimental observation [see Fig. 39(a),(b)]. Increasing the driving amplitude still further does not produce any change in the simulations, as the second harmonic has already become irrelevant. Accordingly, no breakup was found corresponding to Fig. 39(c), which represents the highest experimental driving amplitude. It seems as if the jet profile is severely distorted even two wavelengths before breakup, indicating a strong increase in nonharmonic input, which was not accounted for in the simulations.

Thus we have seen that even small changes in the driving have a significant effect on breakup. Increasing the driving amplitude leads to a transition to symmetric pinching in both simulation and experiment. However, too little is known about the actual experimental driving to draw more definite conclusions.

\section{RELATED PROBLEMS}

For the last section we have selected three more general problems, which stand out not only for their fascinating physics, but also for their technological importance. The dynamics of two-fluid systems governs mixing processes. Here the assumption of a free boundary is abandoned. Shearing forces act on the piece of fluid about to break up, and the dynamics of the outer fluid have to be considered as well. Electrically driven jets are being used for the production of sprays of very small drops of uniform size, and of charged macromolecules in the gas phase. These jets generically appear when a polarizable fluid is placed in a strong electric field. Finally, liquids containing macromolecules are ubiquitous in industrial processing such as fiber spinning. Macromolecules have long time scales associated with their distortion and relaxation, which interfere with the time scales relevant for breakup. This and their highly anisotropic viscosity fundamentally alters the behavior near breakup.

\section{A. Two-fluid systems}

Here we consider the dynamics and breakup of a drop of fluid in another fluid, which is considered to be unbounded. In the case of breakup in air, both the viscosity and the density of the gas are usually negligible, but for fluids the dynamics change. First, the outer fluid has to move with the inner fluid, and second it exerts a force through its shear stresses. This is true even if the outer fluid is at rest, but if in addition there is a nontrivial outer flow, it acts as an external forcing that deforms the drop.

The main interest in the subject comes from the problem of mixing. Figure 42 shows how a single drop is quickly dispersed into a large number of small droplets, which are distributed almost evenly throughout the container. First the drop is stretched into a long filament. Even though it becomes very thin, it remains remarkably stable against capillary instability. Owing to the complicated structure of Lagrangian trajectories even in simple flows, the filament is folded into an intricate structure. In certain parts of the flow the filament finally breaks up into drops under the action of surface tension, and the container is soon filled with tiny drops.

It was G. I. Taylor's idea to break up this extremely complicated phenomenon into a number of simpler ones, two of which he hoped would depend only on the local properties of the flow field, namely, the deformation and stretching of drops and the breakup of long filaments. Only the folding depends on the global structure of the flow, and indeed the chaotic motion of Lagrangian paths is a research subject in its own right, not 

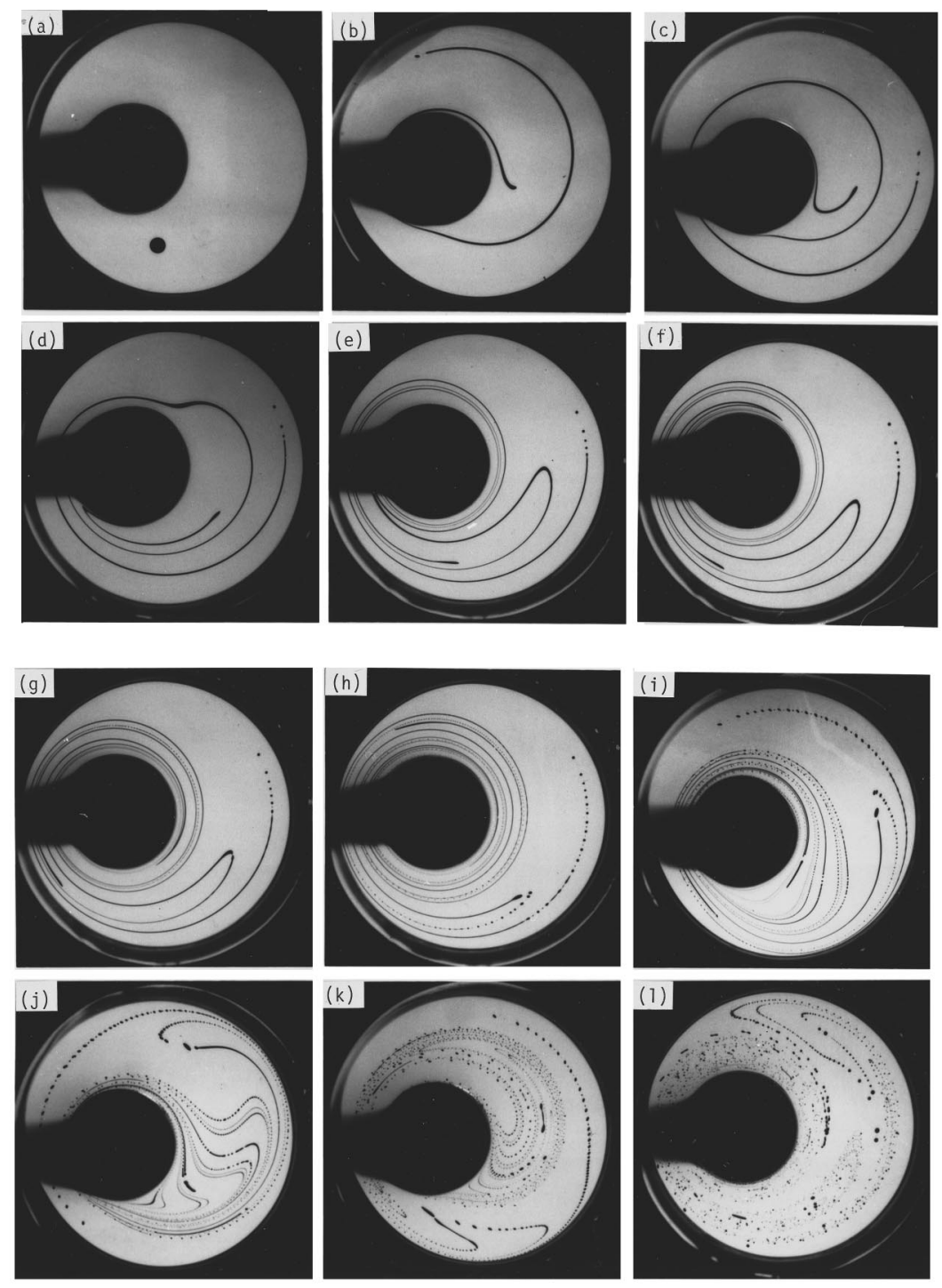

FIG. 42. Dynamics of elongation, folding, and breakup of a drop placed in the globally chaotic flow produced in a time-periodically modulated eccentric journal bearing. The drop is less viscous than the surrounding fluid by a factor of $\lambda=0.067$ (Tjahjadi and Ottino, 1991). Reprinted with permission of Cambridge University Press.

to be considered here. The fundamental approach in all of the following is to look at a single drop or filament and to assume its dimensions to be small compared with the typical scale of the outer flow. Thus the outer flow can be treated in a linear approximation

$$
\mathbf{v}=\boldsymbol{\Gamma} \cdot \mathbf{x}=\frac{1}{2} \boldsymbol{\omega} \times \mathbf{x}+\mathbf{D} \cdot \mathbf{x},
$$

where $\boldsymbol{\omega}$ is the vorticity, $\mathbf{D}$ the deformation tensor, and $\boldsymbol{\Gamma}^{T}$ the velocity gradient tensor. Moreover, in almost all of the work on the problem to date the drop is assumed to be small enough for the Reynolds number to be small in both fluids, so that the Stokes approximation is applicable.

In his first two papers Taylor $(1932,1934)$ looked at the stationary shape and the stability of drops in two prototypical flows. One is a purely extensional flow with zero vorticity, the other a simple shear flow. At a critical flow rate, which depends on the viscosity ratio between the two fluids, the stationary drop becomes unstable and starts to stretch. This situation was investigated by Tomotika (1936), who explained the surprising stability of cylindrical filaments in the process of stretching. When the flow is turned off, or in the context of mixing, if the filament is convected into a region of low strain rate, it is prone to capillary instability, which was investigated in an earlier paper (Tomotika, 1935). The capillary instability and the subsequent nonlinear evolution were investigated both experimentally and numerically by Leal and co-workers, and are summarized in the review paper by Stone (1994).

\section{Stationary shapes}

As suggested by Taylor, we are looking at the case of a linear flow field, either two-dimensional extensional flow

$$
\boldsymbol{\Gamma}=G\left[\begin{array}{ccc}
1 & 0 & 0 \\
0 & -1 & 0 \\
0 & 0 & 0
\end{array}\right],
$$

or simple shear flow 


$$
\boldsymbol{\Gamma}=\frac{G}{2}\left[\begin{array}{lll}
0 & 1 & 0 \\
0 & 0 & 0 \\
0 & 0 & 0
\end{array}\right] .
$$

If the undisturbed drop radius is $a$, then the static problem is governed by the viscosity contrast

$$
\lambda=\frac{\eta^{\prime}}{\eta}
$$

which measures the viscosity of the drop $\eta^{\prime}$ relative to that of the outer fluid $\eta$, and the capillary number

$$
C a=\frac{G \eta a}{\gamma},
$$

which measures the strength of the external shearing forces relative to the surface tension forces. The Reynolds number is assumed to be small and the drop fluid is chosen to be neutrally buoyant, $\rho^{\prime}=\rho$.

For increasingly large capillary numbers, the drop deforms more and more, as measured by the ratio of the half-widths, $L / B$. The outer flow stretches small disturbances on the surface, so the drop is stable against capillary instability even if it is long and thin. The strain rate at which drops can exist in equilibrium is limited rather by the pressure, which builds up at the end of the drop. Here, fluid is being pushed toward the tip, and a pressure gradient maintains the necessary backflow. At some critical capillary number the pressure at the end of the drop overcomes capillary pressure, the drop becomes unstable, and it starts to stretch with the flow. The smaller the viscosity of the drop, the smaller the pressure needed to maintain the backflow, and the more extended the drops become before they start to extend. In extensional flow, for any viscosity ratio there is a critical capillary number that breaks the drop. If vorticity is present, as in the flow (174), drops of high viscosity behave in radically different ways, as discovered by Taylor (1934). For $\lambda \gtrsim 4$ the drop performs a solid-body rotation, thus evading the shearing forces, and no longer breaks up even at the highest capillary numbers. Thus a drop of a high viscosity fluid must be mixed by entirely different means from those of Fig. 42.

Figure 43 shows drops at the critical capillary number for two different viscosity ratios in an extensional flow. At the higher viscosity the drop is still close to spherical at the point of instability, so it can be described in an expansion around this shape. On the other hand, for very long drops of low viscosity ratio, Taylor (1966) proposed an expansion for small $B / L$, very similar to the lubrication expansions of Sec. V. Taylor's ideas were subsequently developed into a fairly complete theory of equilibrium shapes by A. Acrivos and his associates (Barthès-Biesel and Acrivos, 1973; Acrivos and Lo, 1978; Rallison and Acrivos, 1978; Hinch and Acrivos, 1979, 1980).

Barthès-Biesel and Acrivos (1973) carried the expansion for small deformation to second order and used it to predict both drop shapes and critical capillary number at large $\lambda$. Buckmaster (1972) investigated the opposite
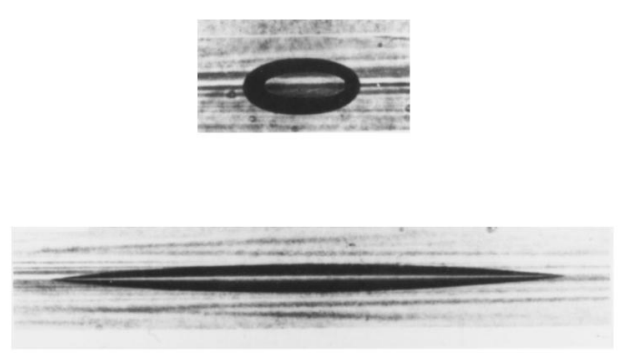

FIG. 43. Two examples of drops in an extensional flow at the critical capillary number. The top picture shows a highly viscous drop $(\lambda=24.5)$, which is still ellipsoidal at the critical capillary number of $C a_{\mathrm{cr}}=0.106$. The bottom picture shows a highly deformed drop with $\lambda=1.05 \times 10^{-3}$ at $C a_{\mathrm{cr}}=0.457$. Note the pointed ends. From Bentley and Leal, 1986b. Reprinted with permission of Cambridge University Press.

limit of an inviscid bubble and found a family of elongated shapes with pointed ends by using slender-body theory. Later, Acrivos and Lo (1978) singled out one shape,

$$
h(z)=\frac{a}{4 C a}\left[1-\left(\frac{z}{20 a C a^{2}}\right)^{2}\right]
$$

as the only stable solution. They also generalized the previous results to viscous drops, which allowed them to find the critical capillary number as a function of $\lambda \ll 1$ :

$$
C a_{\mathrm{cr}} \lambda^{1 / 6}=0.148 .
$$

In particular, this means that an inviscid drop never becomes unstable and can be stretched to arbitrary lengths. Hinch and Acrivos $(1979,1980)$ removed the simplifying assumption of axisymmetry in these calculations, but found the results to be fundamentally the same. The theoretical results were checked against numerical calculations by Rallison and Acrivos (1978) and against experiments by Bentley and Leal (1986a, 1986b). Good agreement was found both in the shapes and in the critical capillary number, as long as the deformation was either small or very large.

The major unsolved problem connected with the theory of stationary shapes concerns the solution (177), valid for very elongated and almost inviscid bubbles. Equation (177) predicts a pointed conical tip at the end of the bubble, in agreement with experiment (see Fig. 43). Thus one would have a singularity of the curvature at the end. However, Buckmaster (1972) used a local analysis to show that conical tips are in fact not a solution of the equations, so the expansion leading to Eq. (177) has to break down near the tip. In light of our analysis of a receding tip in Sec. VI.D this may not be surprising, as lubrication equations should be expected to break down near a tip. Buckmaster (1972) estimated the size of the region where lubrication theory breaks down to be of the order of $\exp \left[-C a^{6}\right]$. In this breakdown lies a tantalizing analogy to two-dimensional Stokes flow, which produces an apparent cusp of a free surface (Joseph et al., 1991). Here the shear needed to form the cusp is produced by two rollers submerged under the surface of a tank of viscous fluid. An exact solu- 


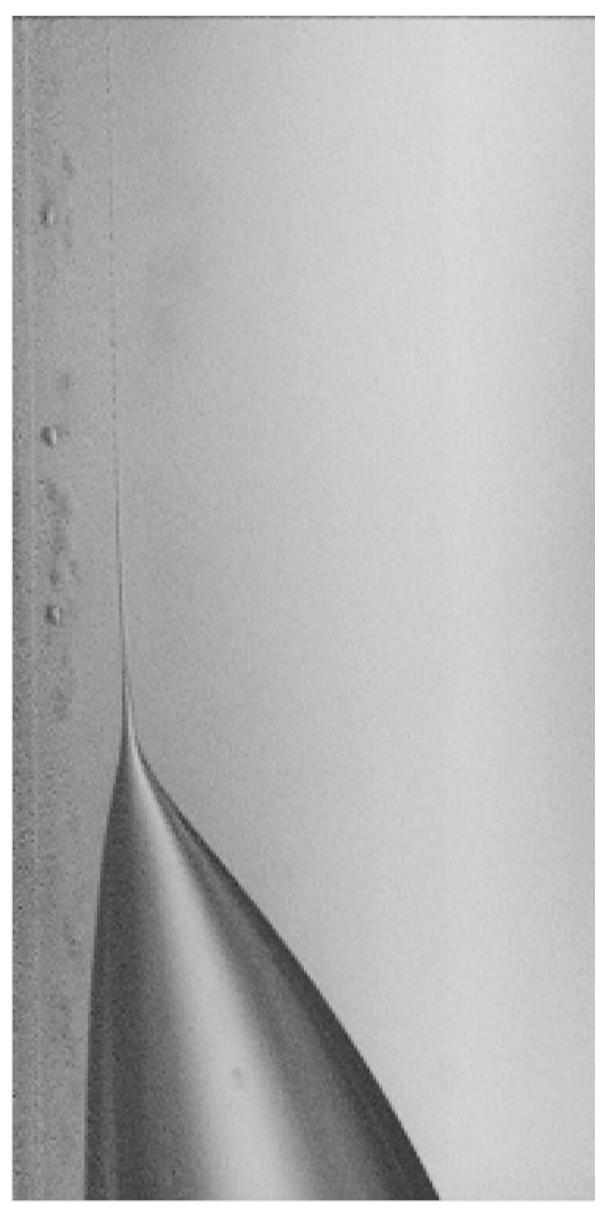

FIG. 44. Tip streaming in a Couette device at a shear rate of $19.5 \mathrm{~s}^{-1}$. Shown is a drop of water in silicone oil which is 1000 times as viscous. To initiate tip streaming, $200 \mathrm{ppm}$ of surfactant has been added. The image width is $0.5 \mathrm{~mm}$ (Leonhard, 1996).

tion of a slightly idealized problem was found (Jeong and Moffatt, 1992), which shows that the cusp singularity is actually regularized by surface tension close to the tip, forming a rounded end with a radius of curvature exponentially small in the capillary number. The same has been suspected by Sherwood (1981) to happen in the present problem, but no solution has been found yet.

But the analogy does not stop here. As the capillary number is increased in the two-roller apparatus, a thin sheet of air emanates from the cusp (Moffatt, 1995), thus entraining air into the fluid. A very similar phenomenon had already been observed by Taylor (1934) in the axisymmetric case and goes by the name of "tip streaming." A thin jet of fluid is ejected from the tip, which soon breaks up into droplets due to the Rayleigh instability, as can be seen in Fig. 44. A theoretical explanation of this phenomenon does not exist. Sherwood (1984) suggests that the tip is unstable owing to nonlinearities in the outer flow, and gives some very clear experimental pictures. de Bruijn (1993) experimentally investigates the influence of surfactants and finds that the appearance of tip streaming crucially depends on the type of fluid used. Most importantly, he finds that tip streaming ceases after a certain period of time, possibly after a surfactant is depleted from the tip. If the drop is left at rest for a while, tip streaming commences. Adding a surfactant provokes tip streaming in fluids that ordinarily show no tip streaming. de Bruijn also observes a variety of hysteresis effects as the shear rate is varied. From this it is clear that surfactants have an important if not essential effect on tip streaming. However, it is not obvious that they are really needed to explain the phenomenon, or whether they just enhance the effect. For example, without surfactant the jet might be too thin to be observed or it only appears at much higher shear rates. In any case, a more complete analysis of the tip region even before the onset of tip streaming seems like an obvious first step towards an understanding.

\section{Breakup}

As mentioned before, the growth of perturbations on a fluid filament that stretches at a uniform rate was investigated by Tomotika (1936). As the filament is stretched, the wavelength of the perturbation increases. Its amplitude relative to the radius of the filament increases by only a finite factor, even as the radius goes to zero. However, this factor may be large if the perturbation is introduced at a later stage of the stretching, where the filament is already thinner. Thus, although the relative growth remains finite in the linear approximation, this may be enough to break the filament. But only when the flow is turned off or the flow rate is substantially reduced does exponential growth set in (Tomotika, 1935; Mikami, Cox, and Mason, 1975). Growth rates and maximum unstable wavelength depend on the viscosity ratio $\lambda$.

As the amplitude of perturbations increases, nonlinear effects become important. They have been studied in a series of papers (Stone, Bentley, and Leal, 1986; Stone and Leal, 1989a, 1989b; Tjahjadi, Stone, and Ottino, 1992) experimentally and in parallel simulations. In addition to breakup driven by the Rayleigh instability, a new type of breakup was observed, called "end pinching." As depicted in Fig. 45, as the filament contracts, two bulbous heads form at the end of the filament; they then separate while the rest of the filament remains intact. Only later does the filament break into many small drops under the action of the Rayleigh instability. For moderately extended filaments, $L / a<20$, end pinching is in fact the dominant effect and the filament contracts to a drop before capillary breakup sets in.

Therefore, to observe capillary breakup without interference from finite size effects, filaments have to be stretched considerably. This was done by Tjahjadi, Stone, and Ottino (1992) using a flow between two concentric cylinders. If the viscosity ratio $\lambda$ is high, then the outer fluid can be neglected and one is back at the physical situation of the previous sections. The final state after the breakup of such a filament is shown in the bottom picture of Fig. 46, with matching simulations of Stokes flow. Note the agreement with Fig. 37, published 140 years earlier. Apart from the main satellite formed by the bulbous neck, another much smaller satellite is 


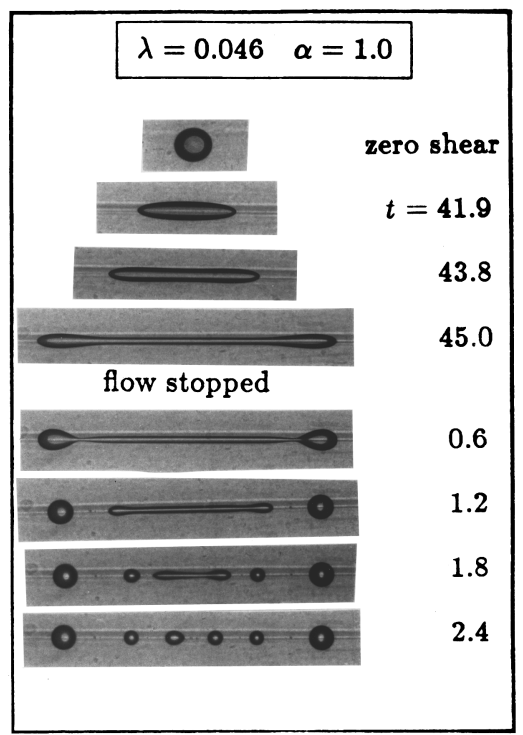

FIG. 45. Extension and subsequent relaxation of a drop at $\lambda=0.046$. After the flow is stopped, the filament first undergoes end pinching and then decays into equally spaced drops corresponding to the most unstable wavelength (Stone, Bentley, and Leal, 1986). Reprinted with permission of Cambridge University Press.

found on either side of it. The reason is that as the fluid pinches at the main drop, a conical neck is left behind. As this neck retracts, it tends to form a bulbous part, which again is prone to pinching.
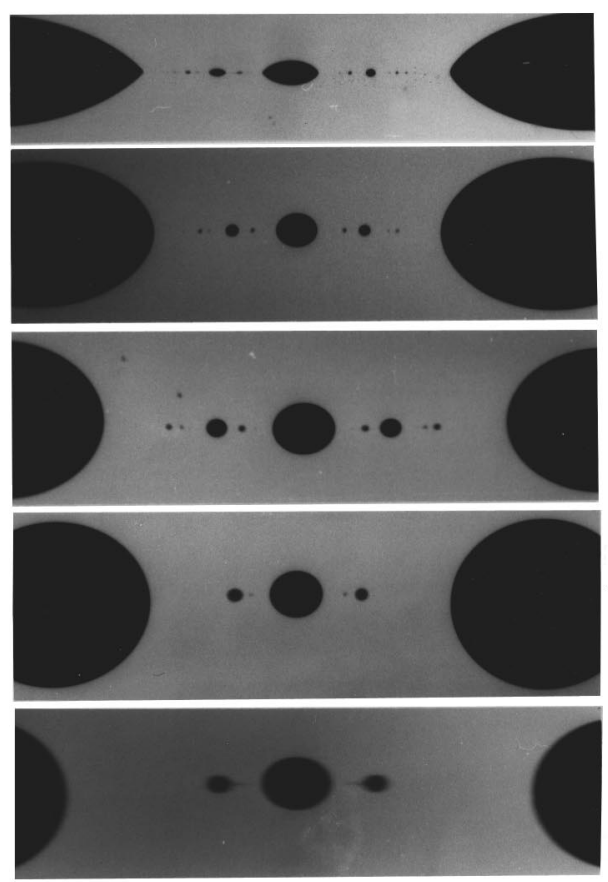

Experiment
As the viscosity ratio is decreased, the final structure becomes ever more intricate, as can be seen by going towards the top of Fig. 46. Even on very small scales, a receding neck has a chance to form a bulbous section before it can completely retract, and the process of pinching becomes self-repeating. For the smallest $\lambda$, satellite drops lie on an intricate fractal structure, the smallest drops observed experimentally having a size of $10 \mu \mathrm{m}$. It seems likely that for finite $\lambda$ there is a cutoff below which no further pinching takes place, but an estimate is not known.

Up to now we have considered only the nonlinear behavior away from the pinch point. As the neck becomes thinner, one expects scale-invariant solutions to appear. However, the force balance cannot be the same as in the free boundary case, and new power laws appear. The reason is that, in the case of a free boundary, viscous stresses are given by $\eta \Delta v / \ell_{z}^{2}$, where $\Delta v$ is a typical velocity scale and $\ell_{z}$ an axial scale. If an outer fluid is present, there must be a boundary layer of the outer fluid over which the velocity at the interface falls off to zero. This is essentially a radial scale, so viscous stresses are now $\eta^{\prime} \Delta v / \ell_{r}^{2}$. Since in the asymptotic solutions with free boundary $\ell_{r}$ goes to zero faster than $\ell_{z}$, viscous stresses due to the outer fluid will eventually dominate, thus changing the force balance. Inevitably, the exponents describing this new solution will also be different. Thus, strictly speaking, even for an outer fluid with very small viscosity $\eta^{\prime}$, the scaling laws will eventually cross over to this new type of scaling.
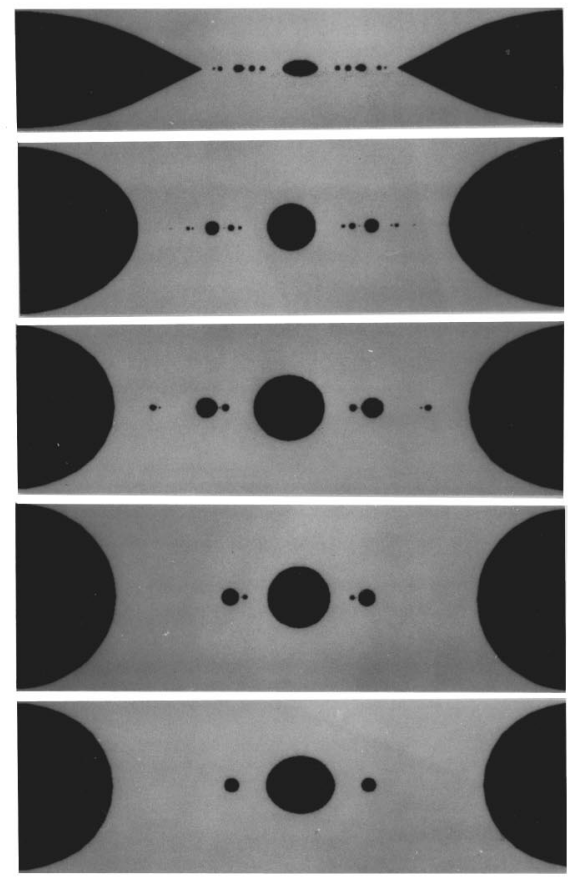

Computation

FIG. 46. Breakup of a filament into satellite and subsatellite drops. As the viscosity ratio $\lambda$ is decreased, the final state of the system has an increasingly complicated fractal structure (Tjahjadi, Stone, and Ottino, 1992). The pictures are, from top to bottom, for $\lambda=0.01,0.067,0.4,1$, and 2.8. Reprinted with permission of Cambridge University Press. 

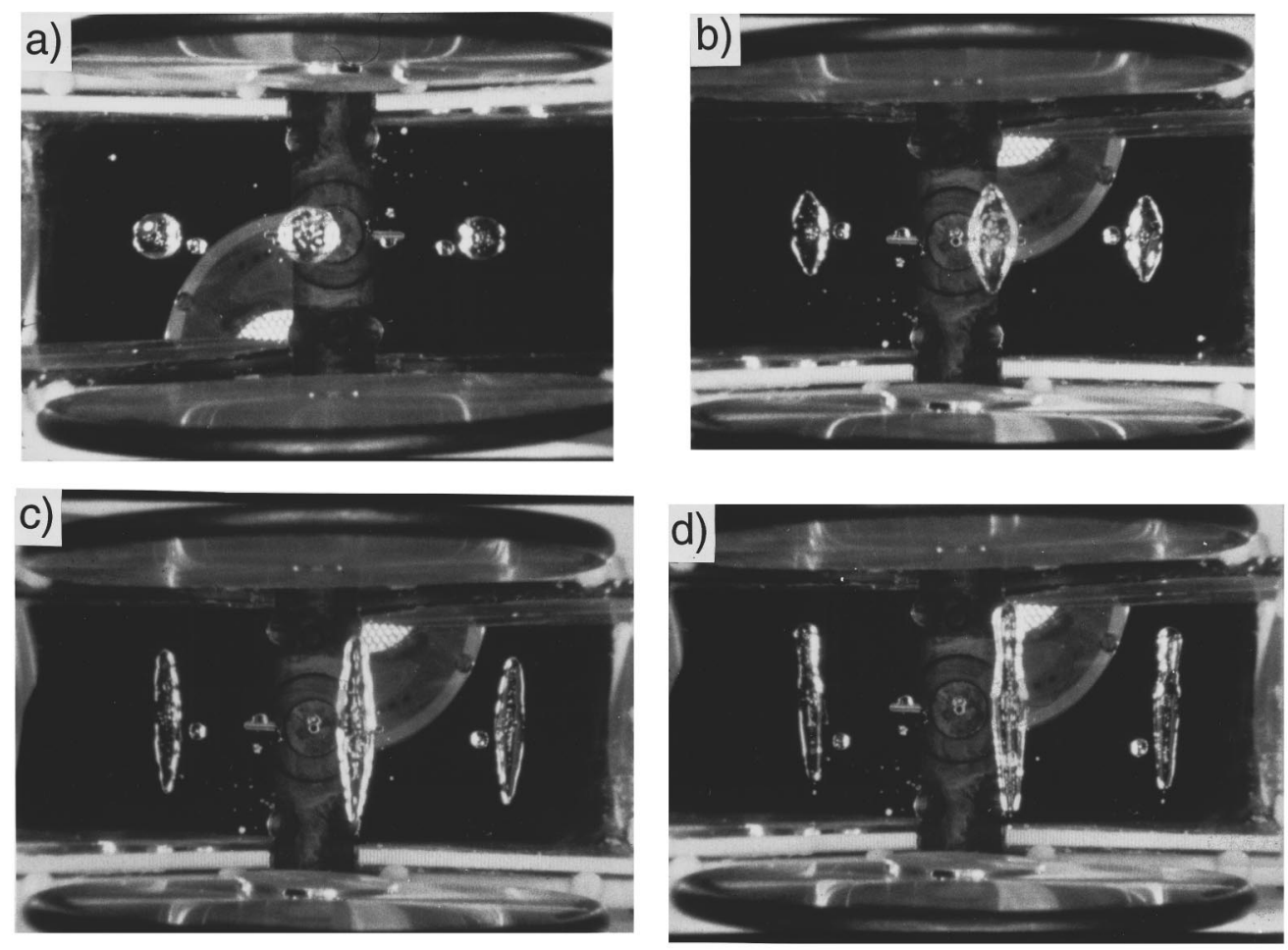

FIG. 47. A drop of water, $1.5 \mathrm{~cm}$ in diameter, subject to a strong electric field of $6 \mathrm{kvolts} / \mathrm{cm}$ in a zero-gravity environment. In (a) the free-floating drop is shown, while the other frames show the drop 3,6, and $9 \mathrm{~ms}$ after the electric field has been turned on (Inculet and Floryan, 1990).

The difficulty in setting up asymptotic equations of motion for this problem lies in the fact that the flow is unbounded. Therefore there is no obvious expansion in a small parameter like the slenderness ratio of the thread. Rather, further knowledge of the boundary layer around the filament is required. One can expect, however, that close to the singularity the contribution from the outer fluid is local as well, in that only flow from close to the point of breaking has to be taken into account. In the case of Stokes flow, some steps have been taken in the direction of "localizing" the contribution from the outer flow (Lister and Stone, 1996; Lister, Stone, and Brenner, 1997), but a self-similar description of the singularity remains a challenging problem.

\section{B. Electrically driven jets}

When a dielectric drop is placed in an electric field, it becomes polarized and the induced surface charges are pulled apart. Thus a more elongated equilibrium shape is reached, which depends on the field strength. Zeleny $(1914,1915,1917)$ discovered that when the electric field strength is raised above a critical value, these shapes become unstable and a conical tip appears at the end of the drop, from which a tiny jet is ejected. Taylor (1964) showed that a cone is a local equilibrium solution in which surface tension and electric forces are balanced. These cones, which have a definite opening angle of $98.6^{\circ}$, are known as Taylor cones. Thus, once more we find a phenomenon in which a surface singularity is formed under the influence of external forces and which then gives birth to a jet or a channel. Similar stretching of a drop and the formation of pointed spikes is observed with ferrofluids in a magnetic field (Cowley and Rosensweig, 1967; Bacri and Salin, 1982).

Two different experimental setups are commonly used. In one, the dielectric fluid is released from a metal capillary tube, which is placed opposite an electrode on a fixed potential relative to the tube. This configuration is used in engineering applications. Gravity can be compensated by the pressure in the tube, and new fluid can be introduced at will. This setup, which readily allows for the formation of electrically driven jets, has gained vast importance in the production of sprays (Bailey, 1988). Recently, the same technique has been used to produce ions of macromolecules in the gas phase and has revolutionized mass spectrometry of such molecules (Fenn et al., 1989).

Despite its great importance, little is known theoretically about the formation of such electrically driven jets or the parameters controlling its properties or stability. For this reason, it is preferable to look at a simpler setup, in which parameters like the geometry of the metal tube, flow rates, or contact angles do not appear. This second common experimental setup consists of an isolated drop placed between two capacitor plates, which produce a uniform electric field. Gravity is eliminated either by using a neutrally buoyant drop (Taylor, 1964) or by placing the drop in a zero-gravity environment (Inculet and Floryan, 1990). Figure 47 shows a se- 


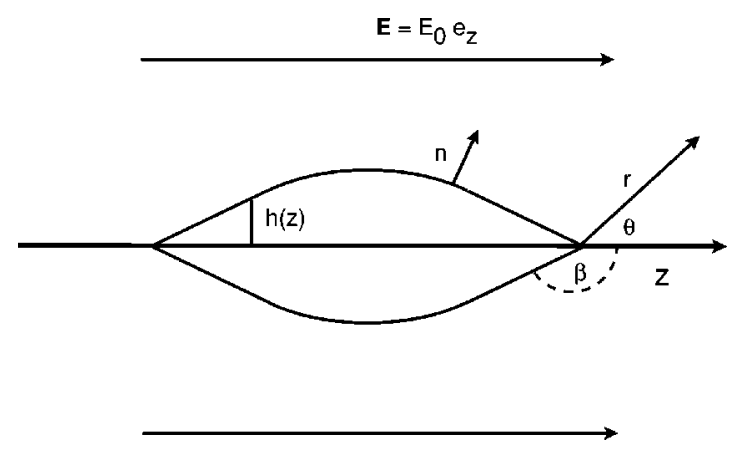

FIG. 48. A cartoon of a fluid drop in a uniform electric field of strength $E_{0}$. At the end the geometry of a cone-shaped tip is shown.

quence of pictures of a water drop in a zero-gravity environment. Frames (b) and (c) show the formation of a Taylor cone after the field has been turned on. In (d) tiny droplets are ejected from the drop, which are visible at the lower end of the drop.

To outline a framework for a possible theoretical description, we set down the corresponding equations for the simplest case of a perfectly conducting fluid, which could serve as a starting point for a more complete dynamical description. Such a dynamic theory, however, has yet to be worked out. For the case of a perfectly conducting fluid, the electric field inside the drop is zero and the outer field $\mathbf{E}$ has only a normal component $E_{n}$ on the surface; see Fig. 48. This leads to an additional normal stress, so the boundary condition for the normal component of the stress tensor is now (Landau and Lifshitz, 1984b)

$$
-\mathbf{n} \boldsymbol{\sigma n}=\gamma\left(\frac{1}{R_{1}}+\frac{1}{R_{2}}\right)+\frac{\epsilon_{0}}{2} E_{n}^{2},
$$

where $\mathbf{n}$ is the outward normal.

The difficulty of the problem resides in calculating the electric field on the surface, which depends nonlocally on the shape of the interface. In particular, using Green's representation theorem, the integral equation for $E_{n}$ is (Miksis, 1981)

$$
2 \pi z=\int_{\partial \Omega} \frac{1}{r}\left[E_{n} / E_{0}-\frac{\partial z}{\partial n}\right] d \sigma+\int_{\partial \Omega} z \partial_{n}\left(\frac{1}{r}\right) d \sigma,
$$

where $E_{0}$ is the applied field strength. To calculate equilibrium shapes, this equation has to be combined with the condition of equilibrium from Eq. (179), namely,

$$
p=\gamma\left(\frac{1}{R_{1}}+\frac{1}{R_{2}}\right)+\frac{\epsilon_{0}}{2} E_{n}^{2},
$$

where $p$ is the pressure in the fluid. Shapes can be calculated by using the additional constraint that the volume of the fluid is conserved as $E_{n}$ is increased:

$$
\pi \int_{z_{-}}^{z_{+}} h^{2}(z) d z=\frac{4 \pi}{3} a^{3},
$$

where $a$ is the radius without electric field and $z_{ \pm}$denote the two ends of the drop.
Equations (180)-(182) uniquely specify the equilibrium shape of the drop. Clearly this shape can depend only on the electric capillary number

$$
C a^{(e l)}=\frac{\epsilon_{0} E_{0}^{2} a}{\gamma} .
$$

Taylor (1964) approximately calculates the elongation of the drop and the critical capillary number necessary to destabilize it. His calculation is based on the approximation that the shape of the drop is spheroidal, in which case the electric field can be calculated exactly. In the opposite limit of strongly deformed drops, Sherwood (1991) performed a slender-body analysis akin to the one used for a drop in an extensional flow (Acrivos and Lo, 1978). The aspect ratio can be calculated as a function of the electric field, but unfortunately the slenderbody approximation is still quite poor at an aspect ratio of about 10 when compared with numerical simulations. A more refined slender-body analysis has recently been performed by Stone, Lister, and Brenner (1997).

Numerical solutions for drop shapes in an electric field were first computed by Miksis (1981), who solved the integral equation (180) numerically and found good agreement with Taylor's results for the elongation of the drop. More recent numerical studies (Sherwood, 1988; Harris and Basaran, 1993) have extended the numerical analysis to higher field strengths, where pointed tips may form. Sherwood (1988) also includes the dynamics of the problem in the Stokes approximation. However, a major drawback of the reported schemes is that they break down close to the tip. Hence they are not able to distinguish reliably between rounded and pointed ends.

Taylor's approach to the problem of a possible coneshaped solution was rather different in that it focused on a local solution with such singular behavior. Regular solutions of the Laplace equation near a cone of opening angle $2(\pi-\beta)$ have the form, using spherical coordinates (Jackson, 1975),

$$
\phi=A r^{\nu} P_{\nu}(\cos \theta)
$$

where $P_{\nu}$ is a Legendre function of order $\nu$ (see Fig. 48). From this potential, the tangential- and normal-field components are

$$
\begin{aligned}
& E_{t}=-\nu A r^{\nu-1} P_{\nu}(\cos \beta), \\
& E_{n}=A r^{\nu-1} \sin \theta P_{\nu}^{\prime}(\cos \beta) .
\end{aligned}
$$

In a conducting fluid the tangential component must vanish, implying $P_{\nu}(\cos \beta)=0$. At the same time, $E_{n}$ must balance surface-tension forces, and the singular part of Eq. (181) becomes

$$
-\frac{\gamma}{r \tan \beta}=\frac{\epsilon_{0}}{2} E_{n}^{2}
$$

so that from Eq. (185b) we have $\nu=1 / 2$. This means that the condition of equilibrium becomes

$$
P_{1 / 2}(\cos \beta)=0,
$$

whose solution is $\beta=130.71^{\circ}$. 


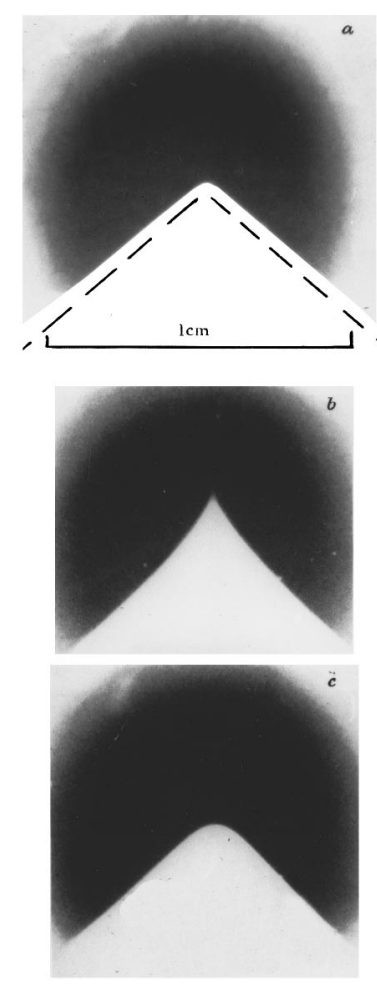

FIG. 49. Three successive frames (in intervals of 1/64 s) showing a drop of water (which appears bright) forming a Taylor cone surrounded by oil. The first frame shows a comparison with the theoretical prediction of the cone angle; the other frames show the drop in two phases of its oscillation around this cone (Taylor, 1964). Reprinted with permission of the Royal Society.

Thus one ends up with a very definite answer, which was confirmed by Taylor in his experiments with a conducting fluid [see Fig. 49(a)], but using electrodes with a shape adjusted to the form of the local solution. Indeed, Eq. (186) requires a definite value of the electric field strength at a given distance from the vertex, and it is not clear under what conditions this local field is consistent with the applied field $E_{0}$ and the shape of the drop. A first step to investigate this problem was taken by $\mathrm{Li}$, Halsey, and Lobkovsky (1994), who computed the energy of a drop with a trial shape, having two pointed ends, in a homogeneous electric field and found it to be lower than that of the corresponding ellipsoidal shape. However, there is no reason to believe that their trial shapes are actually solutions of the equations. The local cone-shaped solutions were incorporated in a numerical scheme for solving Eqs. (180)-(182) by Pantano, Gã̃ ánCalvo, and Barrero (1994). Using the geometry of a capillary tube facing a capacitor plate, they found stationary solutions with pointed tips for a range of values of $C a^{(e l)}$ and $a$.

Li, Halsey, and Lobkovsky (1994) also advance a simple argument that a drop with pointed ends could not correspond to a stable equilibrium. That is, a reduction of the cone angle would increase the field strength, elongating the end even more. Conversely, an increase of the angle would reduce the field strength, so perturbations of that sort would grow as well. This instability is consistent with experimental observations, in which periodic oscillations of the cone angle are observed, the tip being more pointed than the static solution in one phase, and more rounded in the other, see Fig. 49(b),(c). In the pointed phase a jet is ejected from the tip, which is too thin to be resolved in the photograph of Fig. 49.

For other fluids or field strengths, the combined system of a cone and the jet issuing from its tip is stable. Yet it is not known which parameters are controlling this behavior and what dynamical mechanism is responsible for the stability. It has been suggested that a finite conductivity of the fluid is essential for stability (Hayati, Bailey, and Tadros, 1986, 1987). A finite tangential electric field produces shear stresses on the surface that drive a circulatory motion in the fluid, which might be important for stability. Shear stresses on the surface have also been discussed in connection with the stability of the jet away from the apex of the cone (Mestel, 1994). In fact it is another open question originally addressed by Taylor (1969) why an electrically driven jet is far more stable than estimates based on the Rayleigh instability would suggest. Taylor investigated the influence of surface charges and found them to have stabilizing as well as destabilizing effects, depending on wave number. Thus the presence of surface charges alone cannot account for the observed stability.

So far it is not even known what sets the scale of the jet radius. As conductivity increases, meaning that charge relaxation becomes faster relative to the time scale of the fluid motion, the radius of the jet decreases. In the extreme case of liquid metals, the size of the jet decreases to the point where single ions are emitted (Gabovich, 1983). Some phenomenological theories, relating, for example, the mass flux and the current through an electrical jet, have been proposed by Fernández de la Mora and Loscertales (1994). The gas surrounding the Taylor cone is also known to have an important effect (Aguirre-de-Carcer and Fernández de la Mora, 1995).

Nevertheless, despite some advances, the understanding of electrically driven jets remains quite rudimentary. Given the success of one-dimensional models in the description of free jets, it seems promising to include an outer electric field to formulate a minimal dynamic model. Interesting questions are both the approach to the Taylor cone in a strong electric field and the dynamics of the cone-jet system. Again we restrict ourselves to the case of a perfectly conducting fluid, where no model for charge transport has to be considered. The electric field is given solely by electrostatics. A comparison between Eqs. (179) and (19) shows that in that case the pressure as given by Eq. (82) has to be replaced by

$$
p=\gamma\left(\frac{1}{h\left(1+h^{\prime 2}\right)^{1 / 2}}-\frac{h^{\prime \prime}}{\left(1+h^{\prime 2}\right)^{3 / 2}}\right)+\frac{\epsilon_{0}}{2} E_{n}^{2} .
$$

The normal-field component has to be calculated from Eq. (180). Thus in the resulting system (79), (80) all points are nonlocally coupled. Even if this model proves too simple to describe all the experimental effects, and 


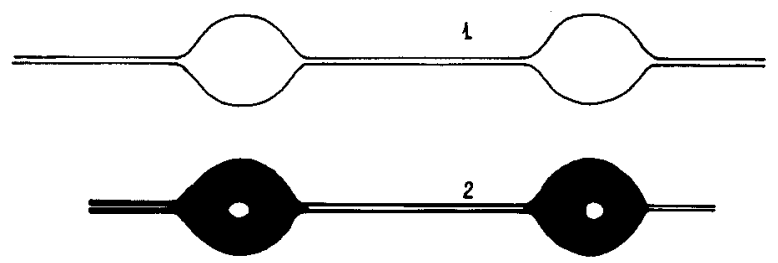

FIG. 50. Beads-on-a-string structure of a jet of $0.02 \%$ aqueous polyoxyethylene WSR-301 solution. The unperturbed radius is $r_{0}=0.027 \mathrm{~cm}$. The theoretical calculation by Yarin (1) is compared with the experiment (2) by A. V. Bazilevskii (Yarin, 1993). Reprinted with permission of Addison Wesley Longman Ltd.

we have mentioned plenty of evidence for this, it will provide a simple starting point for the exploration of the dynamics. Numerical investigations of Eqs. (79), (80), (180), and (188) are under way (Brenner, 1997).

\section{Polymeric liquids}

In this final subsection we turn to liquids that have rheological properties different from the Newtonian fluids described by the Navier-Stokes equation. This happens whenever the fluid contains macromolecules, even if they are present in only minute concentrations. It goes without saying that such fluids are ubiquitous in chemical processing, so it is essential to understand their nonlinear dynamics, which are fundamentally different from those of the Newtonian fluids discussed before. Two properties are responsible for this: First, polymers change the viscosity of the suspension by changing their shape depending on the type of flow. Second, polymers have long relaxation times associated with them, which are easily of the same order as the time scale of the flow and allow the polymers to respond to the flow with a corresponding time delay. Since the polymers are convected with the flow, this makes the equations of motion inherently nonlinear, even if the flow without polymer would be described by linear laws.

The most significant effect in the context of filament breaking is the dramatic increase in viscosity in an extensional flow, which is the typical flow near a pinch singularity, as fluid is pushed away to either side of the pinch point. Specifically, polymers, which are random coils in the absence of flow, are pulled apart and may increase their length by three orders of magnitude in the direction of the extension (Spiegelberg and McKinley, 1996). As a result, the suspension can sustain much greater stresses, and pinching is stopped in regions where polymers are stretched. This "extensional thickening" leads to the characteristic "beads-on-a-string" shape of polymeric jets, as seen in Fig. 50. Some other experimental pictures of polymeric jets are contained in Goldin et al. (1969).

Phenomenological theories incorporating the influence of polymers have been ubiquitous since the early 1950s. In addition, microscopic interpretations and models have been supplied by calculations based on the
Boltzmann equation. Both developments are reviewed extensively in the two-volume monograph by Bird, Armstrong, and Hassager (1987). The validity of these models has been verified in many circumstances of flow regimes where linear approximations of the equations are valid. However, their predictive power in strong flows, where nonlinear effects produce corrections of the same order as that of the basic flow, are a subject of debate. The question is not easy to settle, since the equations are usually very hard to solve even by numerical means, owing to the added nonlinearity and wide range of time scales. Thus for free-surface flows, a quantitative comparison with experiment has never been performed in a regime where the polymer time scale is much longer than the time scale of the flow. The jet flows that are the subject of this review are ideal in this respect, because their extensional character selectively probes the nonlinear properties, and their almost onedimensional behavior makes them both conceptually and computationally simple. In fact, one-dimensional models including viscoelastic effects have been developed in parallel to the Newtonian case (Renardy, 1982; Forest and Wang, 1990, 1994). We shall directly use the one-dimensional description to illustrate the new physics that comes into play.

To this end we add a polymer contribution to Eq. (79) for the velocity field:

$$
\begin{aligned}
\partial_{t} v+v v^{\prime}= & -p^{\prime} / \rho+3 \nu_{s} \frac{\left(h^{2} v^{\prime}\right)^{\prime}}{h^{2}} \\
& +\frac{1}{h^{2}}\left[\left(\sigma_{z} h^{2}\right)^{\prime}-\left(\sigma_{r} h^{2}\right)^{\prime}\right] .
\end{aligned}
$$

Here $\sigma_{z}$ and $\sigma_{r}$ are the lowest-order coefficients in a radial expansion of the diagonal elements of the stress tensor. As the contribution from the solvent has been included explicitly, $\sigma_{z}$ and $\sigma_{r}$ describe only the state of the polymer. The dynamics of this state are accounted for by the equations of motion (Bechtel, Bolinger, Cao, and Forest, 1995),

$$
\begin{gathered}
\partial_{t} \sigma_{z}+v \sigma_{z}^{\prime}-2 v^{\prime} \sigma_{z}=\frac{2 \nu_{p}}{\lambda} v^{\prime}-\frac{\sigma_{z}}{\lambda}-\frac{\alpha}{\nu_{p}} \sigma_{z}^{2}, \\
\partial_{t} \sigma_{r}+v \sigma_{r}^{\prime}+v^{\prime} \sigma_{r}=-\frac{\nu_{p}}{\lambda} v^{\prime}-\frac{\sigma_{r}}{\lambda}-\frac{\alpha}{\nu_{p}} \sigma_{r}^{2},
\end{gathered}
$$

which describe polymers that contribute a Newtonian viscosity $\nu_{p}$ if the fluid moves very slowly. They respond with a time delay of $\lambda$ to the motion of the liquid, as represented by the relaxation term $\sigma / \lambda$ on the right.

It should be noted that this assumption of a single relaxation time $\lambda$ is grossly simplifying, even if the polymers are monodisperse. Rather, one would expect a long chain to have a continuous distribution of time scales, corresponding to various subchains that compose the polymer. In principle, there is no problem in incorporating such a distribution of time scales in the model, but it would violate our fundamental desideratum of simplicity. Usually, one chooses $\lambda$ to be some average of those time scales, but perhaps it is more reasonable to 
assume that strong flows will be dominated by the longest time scale of the system. Recently, other types of viscoelastic fluids have been designed that well approximate the assumption of a single time scale (Rehage and Hoffmann, 1991). Further testing of their properties in strong flows seems highly desirable.

The crucial term in Eq. (190) for $\sigma_{z}$ and $\sigma_{r}$ is the third on the left, which describes the stretching of the polymer as the fluid element containing it elongates. In extensional flow $v^{\prime}$ is positive, so the stress in the axial direction grows, while it decays in the radial direction. Comparing the stretching term with the relaxation term, one finds that exponential growth is possible if

$$
v^{\prime}>\frac{1}{2 \lambda} \text {. }
$$

The dimensionless quantity

$$
\mathrm{De}=\lambda \dot{\gamma}
$$

where $\dot{\gamma}$ is a typical strain rate of the system, is usually called a Deborah number. Thus if De $>1 / 2$, viscoelastic effects will become important, qualifying the problem as a "strong flow."

The most controversial terms are the quadratic ones on the right, whose physical meaning is to limit the polymeric viscosity as the polymer is stretched to its full length. This particular nonlinearity was proposed by Giesekus (1982), and the three-dimensional version of Eqs. (189) and (190) is referred to as the Giesekus model. Some nonlinear term is necessary to avoid the unphysical assumption of polymers stretching to arbitrary lengths. In fact Renardy (1994) shows that the model (189), (190), with $\alpha=0$ and in the absence of inertia, will show no thread breakup at all, which is clearly unphysical. The problem of the quadratic term is that it gives polymer extensions proportional to the strain rate, while in reality it should be limited by the chain length. In the absence of an obvious better choice, we shall not dwell on the subject here. Some other possibilities are discussed by Hinch (1977). To demonstrate that these one-dimensional models make sense, the experimental picture (see Fig. 50) of a polymeric jet is compared with a calculation by Yarin (1993). His one-dimensional model is very similar to Eqs. (189) and (190), except that the nonlinear terms in (190) are based on the theory by Hinch (1977). The almost cylindrical portions of the jet are quite well reproduced.

The mathematical properties of one-dimensional models have been studied extensively (Forest and Wang, 1990; Bechtel, Forest, and Lin, 1992; Renardy, 1994; Bechtel, Bolinger, Cao, and Forest, 1995) and simulations under idealized conditions have been performed as well (Forest and Wang, 1994; Renardy, 1995). By using only the leading-order curvature contribution, and for $\alpha=\nu_{s}=0$, a linearization reveals that the model may enter a regime where it is ill posed (Forest and Wang, 1990). Since surface gradients become quite large, this may well be the result of neglecting regularizing terms in the curvature (Renardy, 1995). The ab- sence of a solvent and the infinite extensibility of the polymer are also unphysical assumptions, which may cause mathematical problems. Clearly there is a need for a better understanding of the mathematical significance of the different terms in Eq. (190). In a sense, there is no reason why the one-dimensional approximation in itself should be a cause for catastrophic instability, while no problem occurs in the full equations.

Clearly, it is most desirable to perform some quantitative test of viscoelastic models in strong flows. Sridhar et al. (1991) report an experiment in the liquid-bridge geometry described in Sec. II.C, where one plate moves with a velocity increasing exponentially in time and gravity points in the direction of the axis. This ensures a constant strain rate, thus selectively probing polymer stretching over a given time scale. However, the strain rates were not high enough to qualify for a strong flow. Shipman, Denn, and Keunings (1991) performed a finite-element study of the same experiment, but used only the minimum radius of the bridge for comparison.

Recently, the same experiment has been performed at much higher Deborah numbers of up to 13 (Spiegelberg, Ables, and McKinley, 1996). Figure 51 shows a comparison of a Newtonian liquid with a polymeric liquid at a Deborah number of 3.6. At low shear rates, the properties of both fluids are the same. The viscosity is very high, so that inertial effects and even surface tension forces are small during most of the experiment. The Hencky strain $\epsilon$ shown below each frame is the natural logarithm of the factor by which the plate spacing has increased. Initially, the evolution of the profiles of both liquids are very similar. But as the plate spacing increases by more than a factor of 8 , polymers are sufficiently stretched to have a significant effect. As a result, the profile of the polymeric fluid develops the typical columnar shape already observed in the case of purely surface-tension-driven breakup. At $\epsilon=4.5$, the column is resting on an almost flat surface, which has even developed a small dip. The last frame shows a new instability, in which the fluid starts to break up into individual threads.

This set of experiments is ideally suited for a comparison with the lubrication models explained above. The fluids are well characterized and thus allow for a quantitative test of different viscoelastic models in the strongflow regime. Their simplicity nurtures hope for an understanding of the fundamental physics of viscoelastic liquids, such as the columnar structure. A host of experimental data are available, most notably the surface profiles and the force on the endplates. The latter is a measure of the stress supported by the polymers and thus directly probes nonlinear terms in Eq. (190).

The elastic instability seen in the last frame of Fig. 51 is, of course, beyond the description possible with a onedimensional model. It has been studied in more detail by Spiegelberg and McKinley (1996). Even the dip appearing in the next-to-last frame signals trouble, as the model proposed here is not able to describe overturning. At best, the model will be able to describe the experiment up to the point where the dip occurs. One should 

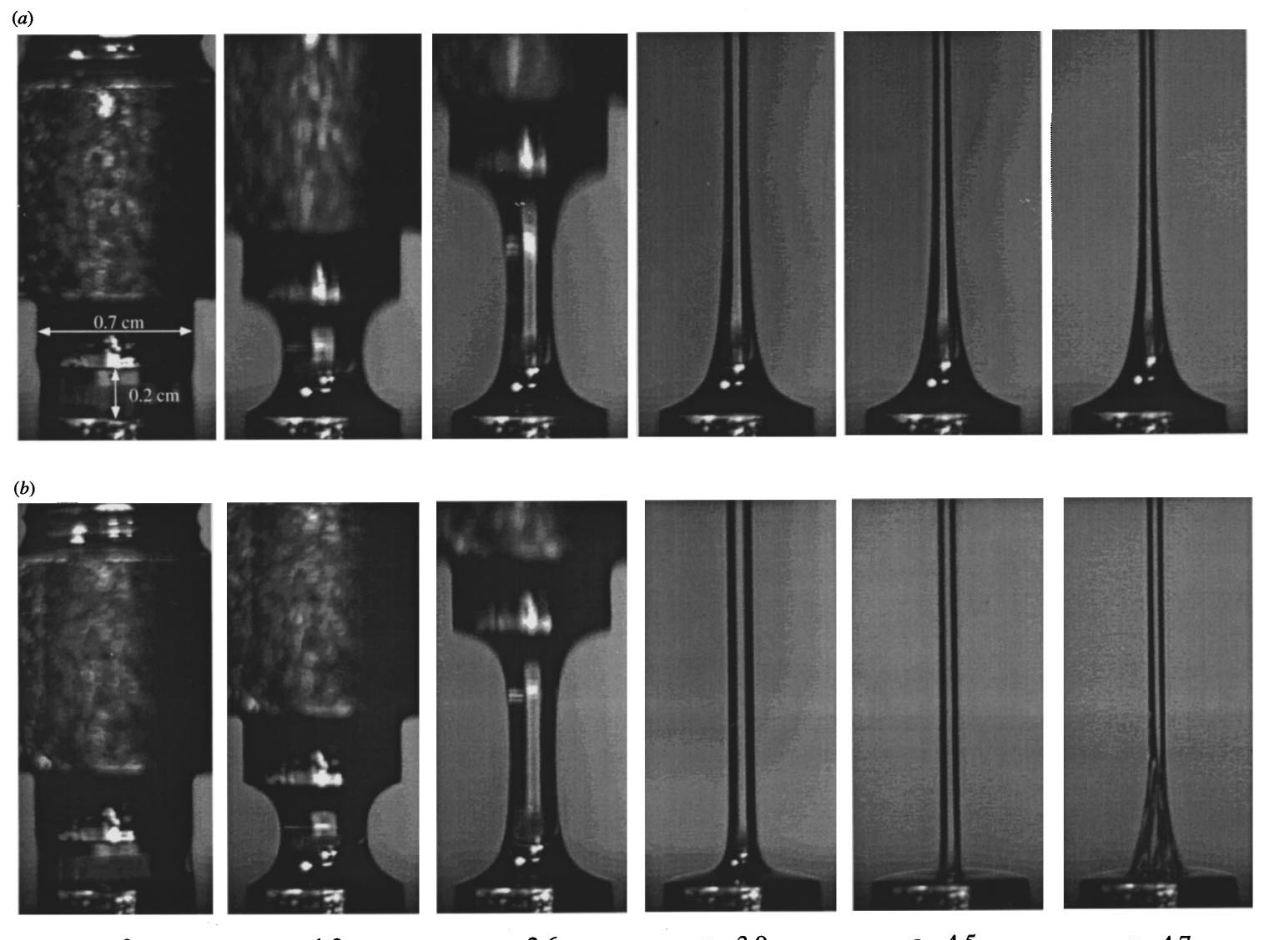

$\varepsilon=2.6$

$\varepsilon=3.9$

$\varepsilon=4.5$

$\varepsilon=4.7$

FIG. 51. Two fluid filaments undergoing an exponential elongational stretch. Corresponding Hencky strains are shown. A Newtonian polybutene oil (a) is compared to a polymeric fluid (b). The ratio of the polymeric time scale and the time scale of the flow is $\mathrm{De}=3.6$.

remember, however, that the polymer is subjected to quite extreme conditions in this particular run. Nevertheless, it would be extremely desirable to extend lubrication models so as to describe overturning. This is a subject that has never been addressed in the literature, in spite of its obvious significance. It remains one of the most fundamental unresolved issues of the field.

\section{OUTLOOK}

One-dimensional approximations of the NavierStokes equation driven by surface tension have led to a fairly complete description of the neighborhood of the point of breakup. It is among the great surprises of this research that the same simple models also yield quantitatively correct predictions far away from breakup. The potential of this observation has only begun to be explored, as it will make fine-scale detail of flow problems accessible by numerical means, which are too complicated to be computed from direct simulations of the Navier-Stokes equation. However, to make full use of the technological applications of one-dimensional modeling, the theory has to be extended in several directions. One example is the inclusion of an outer fluid, which will be important for jet atomization and mixing processes. Non-Newtonian fluids and additional types of forcing, like electric or magnetic fields, mentioned in the previous section, are other examples.

In all applications, seemingly simple flow configurations may occur in which the lubrication approach fails. Such configurations occur in the neighborhood of points where the surface profile is not unique or near tips, where the slope becomes infinite. This remains one of the most fundamental problems to be addressed by theory, and it is of the greatest practical importance as well. A possible solution lies in the choice of appropriate coordinate systems, which adapt to the flow. In a slightly different context, this idea has already been used by Yarin (1993) to generalize the slender-jet approach to nonaxisymmetric situations. The centerline of the jet is allowed to be an arbitrary curve in space, around which the slender jet expansion is performed. An equation of motion for the position of the centerline closes the system. Thus the jet may be bending in response to aerodynamic forces or buckling when it falls onto a flat surface.

Complementary to further development of onedimensional models, one needs more accurate experimental and numerical tests of their validity, in particular regarding the flow field. Marschall's (1985) snapshots of material lines of the flow indicate that complicated circular motions may occur, while the surface hardly changes. This suggests that it is more fruitful to think of the velocity field in one-dimensional models as the average over the cross section, which is the point of view adopted in Sec. V.B. In cases where more accurate information on the flow field is needed, one could combine full Navier-Stokes simulations with lubrication approximations in regions where a full simulation is too costly. As indicated in Sec. V, higher-order expansions are available to improve on the error systematically. 
Very close to breakup, when the bridge between the two halves of fluid is of the order of molecules, one needs to go beyond the approximation of continuum mechanics used throughout this review. If the viscosity is high, fluctuations need to be included while the fluid thread is still macroscopic, and breakup patterns become very complicated. In either case moleculardynamics (MD) simulations may provide useful information complementary to hydrodynamic calculations. While in the case of wall-bounded flows layers of 10 molecules are sufficient for a fully quantitative comparison between MD simulations and hydrodynamics (Koplik, Banavar, and Willemsen, 1989), the minimum number of molecules needed for hydrodynamics to apply is not clear for free surfaces. Unfortunately, even the most recent simulations (Koplik and Banavar, 1993) are not quite large enough to show a complete transition from a single cylinder of fluid to two separate pieces. Using effective interactions between water molecules, Greenspan (1993) shows pictures of the bifurcation of a water drop falling from a ceiling under gravity. But clearly, in view of rapidly developing computer capabilities, the gap between MD simulations and hydrodynamics of free surfaces will soon narrow considerably.

Although the problem of inviscid or almost inviscid flow stood at the beginning of both the theoretical (Keller and Miksis, 1983) and experimental (Peregrine, Shoker, and Symon, 1990) study of the pinch singularity, it remains the least understood. The reason lies in the subtle interplay between the pinch singularity and another singularity of the inviscid equations, which causes infinitely steep gradients to occur, as explained in Sec. VII.B. This makes viscosity become important even if it is arbitrarily small, and may cause a breakdown of hydrodynamics before breakup occurs (Brenner et al. 1997). Only in the last stage of pinching, when the minimum height is of the order of the viscous scale, does the universal scaling solution set in, where viscous forces are balanced by surface tension. Because of the practical importance of low-viscosity fluids like water, a description of the almost inviscid transient region is particularly desirable, for example, to understand the formation of satellite drops. In view of its simplicity, low-viscosity pinching may also serve as a paradigm for other highReynolds-number flows such as turbulence (Frisch, 1995), which generically show a great disparity between the length scales on which they are driven and the scales where the flow is smoothed out by viscosity. A particularly interesting possibility is the emergence of anomalous scaling exponents for pinching at low viscosities, which replace the dimensional scaling (Barenblatt, 1996) eventually observed on scales where viscosity becomes dominant.

In many other problems of hydrodynamics singularities develop, and only a few of them have been understood in detail. Examples are the tips of drops in electric fields or in shear flows, mentioned in Sec. VIII. Singularities of the three-dimensional Euler equation may be regarded as the breakup of vortex lines or sheets (Pomeau, 1994). In the case of two-dimensional breakup, as in a Hele-Shaw cell, the form of the singularity depends sensitively on the initial condition (Almgren, Bertozzi, and Brenner, 1996). It is not known under which circumstances a singularity is "universal," as in the case of three-dimensional breakup, or when large-scale information is still present at arbitrarily small scales. What is needed most is a more unified description of these different types of singularities, of which the subject of this review is only one example.

In the context of free-surface flows, another singularity that has never been looked at occurs when two pieces of fluids merge. Some experimental work (Bradley and Stow, 1978) and numerical work (Greenspan and Heath, 1991; Koplik and Banavar, 1992; Lafaurie et al., 1994; Nobari, Jan, and Tryggvason, 1996) provides some hints, but the resolution is insufficient to observe much of the extremely rapid motion after two surfaces touch. The surface is singular initially, so everything is again dominated by surface-tension forces, and the motion can be expected to be universal. A solution of this problem would complete the description of singularities of freesurface flows, being able to separate a piece of fluid into two and join the two pieces together again.

\section{ACKNOWLEDGMENTS}

I was introduced to the subject of drop formation by my thesis advisor, Siegfried Grossmann. But only after some more admonitions by Leo Kadanoff did I take up my own research on the subject. This research got off to a good start due to my collaboration with Todd Dupont, who also taught me most of what I know about numerical methods, and continues to be an inexhaustible source of advice. Over the years I enjoyed collaboration and a constant and fruitful exchange with Michael Brenner. Tomasz Kowalewski designed experiments to test my theory and contributed many interesting discussions and critical remarks. A happy coincidence brought about by this research is also the collaboration with my friend Jens Hoppe. Among the many other people I was fortunate enough to interact with in the course of this work are Hartwig Brand, Peter Constantin, Gareth McKinley, Keith Moffatt, Sidney Nagel, Mike Shelley, Martin Smock, Howard Stone, and Stephane Zaleski. Many other collaborations were made possible by the hospitality of the Department of Mathematics and the James Franck Institute of the University of Chicago during summer months. I am also grateful to the theoretical physics people at the University of Essen, and in particular to Robert Graham, for their support and their patience. Michael Brenner and Howard Stone gave me invaluable advice on the manuscript, and Reuben Schulkes made helpful comments on the section on numerical methods. Finally, this review has been enlivened by many pictures illustrating the intricacy and beauty of free-surface flows. R. Schulkes and S. Zaleski contributed pictures of their simulations. Since experiments are what keeps physics alive, I am particularly happy about the photographs contributed by R. Donnelly, J. Floryan, 
G. Jameson, T. Kowalewski, G. Leal, H. Leonhard, S. Nagel, J. Ottino, S. Spiegelberg, H. Stone, and A. Yarin.

\section{REFERENCES}

Acrivos, A., and T. S. Lo, 1978, J. Fluid Mech. 86, 641.

Aguirre-de-Carcer, I., and J. Fernández de la Mora, 1995, J. Colloid Interface Sci. 171, 512.

Almgren, R., A. Bertozzi, and M. P. Brenner, 1996, Phys. Fluids 8, 1356.

Altschuler, S., S. B. Angenent, and Y. Giga, 1995, J. Geom. Anal. 5, 293.

Amit, D. J., 1978, Field Theory, The Renormalization Group and Critical Phenomena (McGraw-Hill, New York).

Ashgriz, N., and F. Mashayek, 1995, J. Fluid Mech. 291, 163.

Bacri, J. C., and D. Salin, 1982, J. Phys. (France) Lett. 43, L649.

Bailey, A. G., 1988, Electrostatic Spraying of Liquids (Wiley, New York).

Baker, G. R., D. I. Meiron, and S. A. Orszag, 1980, Phys. Fluids 23, 1485.

Barenblatt, G. I., 1996, Scaling, Self-Similarity, and Intermediate Asymptotics (Cambridge University, Cambridge, England).

Barthès-Biesel, D., and A. Acrivos, 1973, J. Fluid Mech. 61, 1. Bar-Ziv, R., and E. Moses, 1994, Phys. Rev. Lett. 73, 1392.

Bechtel, S. E., K. Bolinger, G. Cao, and M. G. Forest, 1995, SIAM (Soc. Ind. Appl. Math.) J. Appl. Math. 55, 58.

Bechtel, S. E., C. D. Carlson, and M. G. Forest, 1995, Phys. Fluids 7, 2957.

Bechtel, S. E., M. G. Forest, and K. J. Lin, 1992, SAACM 2 , 59.

Becker, E., W. J. Hiller, and T. A. Kowalewski, 1991, J. Fluid Mech. 231, 189.

Becker, E., W. J. Hiller, and T. A. Kowalewski, 1994, J. Fluid Mech. 258, 191.

Bender, C. M., and S. A. Orszag, 1978, Advanced Mathematical Methods for Scientists and Engineers (McGraw-Hill, New York).

Bensimon, D., L. P. Kadanoff, S. Liang, B. I. Shraiman, and C. Tang, 1986, Rev. Mod. Phys. 58, 977.

Bentley, B. J., and L. G. Leal, 1986a, J. Fluid Mech. 167, 219. Bentley, B. J., and L. G. Leal, 1986b, J. Fluid Mech. 167, 241. Berger S. A., 1988, SIAM (Soc. Ind. Appl. Math.) J. Appl. Math. 48, 973.

Bertozzi, A. L., M. P. Brenner, T. F. Dupont, and L. P. Kadanoff, 1994, in Trends and Perspectives in Applied Mathematics, Applied Mathematics Series, Vol. 100, edited by L. Sirovich (Springer, New York), p. 155.

Bidone, G., 1823, Mem. Accad. Sci. di Torino 27, 83.

Bird, R. B., R. C. Armstrong, and O. Hassager, 1987, Dynamics of Polymeric Liquids, Volume I: Fluid Mechanics; Volume II: Kinetic Theory (Wiley, New York).

Bogy, D. B., 1978a, J. Appl. Mech. 45, 469.

Bogy, D. B., 1978b, Phys. Fluids 21, 190.

Bogy, D. B., 1979a, Phys. Fluids 22, 224; 23, 421.

Bogy, D. B., 1979b, Annu. Rev. Fluid Mech. 11, 207.

Bogy, D. B., S. J. Shine, and F. E. Talke, 1980, J. Comput. Phys. 38, 294.

Bohr, N., 1909, Philos. Trans. R. Soc. London, Ser. A 209, 281. Bousfield, D. W., R. Keunigs, G. Marrucci, and M. M. Denn, 1986, J. Non-Newton. Fluid Mech. 21, 79.
Bradley, S. G., and C. D. Stow, 1978, Philos. Trans. R. Soc. London, Ser. A 287, 635.

Brenner, M. P., 1997, private communication.

Brenner, M. P., J. Eggers, K. Joseph, S. R. Nagel, and X. D. Shi, 1997, Phys. Fluids 9, 1573.

Brenner, M. P., J. R. Lister, and H. A. Stone, 1996, Phys. Fluids 8, 2827.

Brenner, M. P., X. D. Shi, and S. R. Nagel, 1994, Phys. Rev. Lett. 73, 3391.

Brosa, U., S. Grossmann, and A. Müller, 1990, Phys. Rep. 197, 167.

Buckmaster, J. D., 1972, J. Fluid Mech. 55, 385.

Chandrasekhar, S., 1961, Hydrodynamic and Hydromagnetic Stability (Oxford University, New York/London). Reprinted 1981 (Dover, New York).

Chaudhary, K. C., and T. Maxworthy, 1980a, J. Fluid Mech. 96, 275.

Chaudhary, K. C., and T. Maxworthy, 1980b, J. Fluid Mech. 96, 287.

Chaudhary, K. C., and L. G. Redekopp, 1980, J. Fluid Mech. 96, 257.

Cline, H. E., and T. R. Anthony, 1978, J. Appl. Phys. 49, 3203. Constantin, P., 1992, private communication.

Constantin, P., T. F. Dupont, R. E. Goldstein, L. P. Kadanoff, M. J. Shelley, and S. M. Zhou, 1993, Phys. Rev. E 47, 4169.

Cowley, M. D., and R. E. Rosensweig, 1967, J. Fluid Mech. 30, 671.

Crane, L. S., S. Birch, and P. O. McCormack, 1964, Brit. J. Appl. Phys. 15, 743.

Dafermos C. M., 1987, SIAM (Soc. Ind. Appl. Math.) J. Math. Anal. 18, 409.

Da-Riva, I., 1981, in Applications of Space Development, XXXI International Astronautical Congress, edited by L. G. Napolitano (Pergamon, New York), p. 69.

de Bruijn, R. A., 1993, Chem. Eng. Sci. 48, 277.

de Laplace, P. S., 1805, Méchanique Celeste, Supplément au Xieme Livre (Courcier, Paris).

Deckelnick, K., 1997, "Weak Solutions of the Curve Shortening Flow," Calc. Var., in press.

Dewynne, J. N., P. D. Howell, and P. Wilmott, 1994, Q. J. Mech. Appl. Math. 47, 541.

Dold, J. W., 1992, J. Comput. Phys. 103, 90.

Dommermuth, D. G., and D. K. P. Yue, 1987, J. Fluid Mech. 178, 195.

Donnelly, R. J., and W. Glaberson, 1966, Proc. R. Soc. London, Ser. A $290,547$.

Dupont, T. F., 1993, private communication.

Edgerton, H. E., 1977, in Stopping Time: The Photographs of Harold Edgerton, edited by Gus Kayafas (Abrams, New York, 1987).

Edgerton, H. E., E. A. Hauser, and W. B. Tucker, 1937, J. Phys. Chem. 41, 1029.

Eggers, J., 1993, Phys. Rev. Lett. 71, 3458.

Eggers, J., 1995a, Phys. Fluids 7, 941.

Eggers, J., 1995b, "Nonlinear stability in one-dimensional models," unpublished.

Eggers, J., 1995c, "Satellite formation in one-dimensional models," unpublished.

Eggers, J., 1997, "Singularities in droplet pinching without viscosity," Los Alamos preprint No. chao-dyn/9705006.

Eggers, J., and T. F. Dupont, 1994, J. Fluid Mech. 262, 205.

Entov, V. M., and A. L. Yarin, 1984, J. Fluid Mech. 140, 91. 
Fenn, J. B., M. Mann, C. K. Meng, S. F. Wong, and C. M. Whitehouse, 1989, Science 246, 64.

Fernández de la Mora, J., and I. G. Loscertales, 1994, J. Fluid Mech. 260, 155.

Forest, M. G., and Q. Wang, 1990, J. Theor. Comp. Fluid Dyn. 2, 1.

Forest, M. G., and Q. Wang, 1994, SIAM (Soc. Ind. Appl. Math.) J. Appl. Math. 54, 996.

Frankel, I., and D. Weihs, 1985, J. Fluid Mech. 155, 289.

Frisch, U., 1995, Turbulence (Cambridge University, Cambridge, England).

Fromm, J. E., 1984, IBM J. Res. Dev. 28, 322.

Gabovich, M. D., 1983, Usp. Fiz. Nauk 140, 137 [Sov. Phys. Usp. 26, 447 (1983)].

García, F. J., and A. Castellanos, 1994, Phys. Fluids 6, 2676.

Gaudet, S., G. H. McKinley, and H. A. Stone, 1996, Phys. Fluids 8, 2568.

Giesekus, H., 1982, J. Non-Newton. Fluid Mech. 11, 69.

Giga, Y., and R. V. Kohn, 1989, Commun. Pure Appl. Math. 42, 845 .

Gillette, R. D., and D. C. Dyson, 1971, Chem. Eng. 2, 44.

Goedde, E. F., and M. C. Yuen, 1970, J. Fluid Mech. 40, 495.

Goldin, M., J. Yerushalmi, R. Pfeffer, and R. Shinnar, 1969, J.

Fluid Mech. 38, 689.

Gradshteyn, I. S., and I. M. Ryzhik, 1980, Table of Integrals, Series, and Products (Academic, New York).

Grauer, R., and T. C. Sideris, 1991, Phys. Rev. Lett. 67, 3511. Green, A. E., 1976, Int. J. Eng. Sci. 14, 49.

Green, A. E., and N. Laws, 1966, Proc. R. Soc. London, Ser. A 293, 145.

Greenspan, D., 1993, System Analysis Modelling Simulation, Vol. 11 (Gordon and Breach, New York), p. 87.

Greenspan, D., and L. F. Heath, 1991, J. Phys. D 24, 2121.

Grossmann, S., and A. Müller, 1984, Z. Phys. B 57, 161.

Grundy, R. E., 1983, IMA J. Appl. Math. 30, 209.

Grundy, R. E., and V. A. Bell, 1993, Proc. R. Soc. London, Ser. A 441, 641.

Guderley, A., 1942, Luftfahrt-Forsch. 19, 302.

Gueyffier, D., and S. Zaleski, 1997, "Simulations of colliding drops with SURFER," unpublished.

Guthrie, F., 1864, Proc. R. Soc. London 13, 444.

Haenlein, A., 1931, Forsch. Geb. Ingenieurwes. 2, 139.

Hagen, G., 1849, Bericht über die zur Bekanntmachung geeigneten Verhandlungen der preussischen Akademie der Wissenschaften (Akademie-Verlag, Berlin), p. 281.

Happel, J., and H. Brenner, 1983, Low Reynolds Number Hydrodynamics (Nijhoff, The Hague).

Harkins, W. D., and F. E. Brown, 1919, J. Am. Chem. Soc. 41, 499.

Harris, M. T., and O. A. Basaran, 1993, J. Colloid Interface Sci. 161, 389.

Hauser, E. A., H. E. Edgerton, B. M. Holt, and J. T. Cox, Jr., 1936, J. Phys. Chem. 40, 973.

Hayati, I., A. I. Bailey, and T. F. Tadros, 1986, Nature (London) 319, 41.

Hayati, I., A. I. Bailey, and T. F. Tadros, 1987, J. Colloid Interface Sci. 117, 205.

Henderson, D. M., W. G. Pritchard, and L. B. Smolka, 1997, "On the pinch-off of a pendant drop of a viscous fluid," Pennsylvania, State University preprint.

Hiller, W. J., and T. A. Kowalewski, 1989, Opt. Eng. (Bellingham) 28, 197.

Hinch, E. J., 1977, Phys. Fluids 20, S22.
Hinch, E. J., and A. Acrivos, 1979, J. Fluid Mech. 91, 401.

Hinch, E. J., and A. Acrivos, 1980, J. Fluid Mech. 98, 305.

Hirt, C. W., and B. D. Nichols, 1981, J. Comput. Phys. 39, 201.

Hoppe, J., 1992, Lectures on Integrable Systems (Springer,

Berlin).

Hoppe, J., 1995, private communication.

Huisken, G., 1991, J. Diff. Geom. 31, 285.

Inculet, I. I., and J. M. Floryan, 1990, Int. J. Microgr. Res. Appl. 3, 148.

Jackson, J. D., 1975, Classical Electrodynamics, 2nd edition (Wiley, New York).

Jeong, J.-T., and H. K. Moffatt, 1992, J. Fluid Mech. 241, 1.

Johnson, G. R., E. Marschall, and J. H. Esdorn, 1985, Rev. Sci. Instrum. 56, 264.

Johnson, M., R. D. Kamm, L. W. Ho, A. Shapiro, and T. J. Pedley, 1991, J. Fluid Mech. 233, 141.

Joseph, D. D., J. Nelson, M. Renardy, and Y. Renardy, 1991, J. Fluid Mech. 223, 383.

Keller, J. B., 1983, Phys. Fluids 26, 3451.

Keller, J. B., A. King, and L. Ting, 1995, Phys. Fluids 7, 226.

Keller, J. B., and M. J. Miksis, 1983, SIAM (Soc. Ind. Appl. Math.) J. Appl. Math. 43, 268.

Keller, J. B., S. I. Rubinow, and Y. O. Tu, 1973, Phys. Fluids 16, 2052.

Kessler, D. A., J. Koplik, and H. Levine, 1988, Adv. Phys. 37, 255.

Keunings, R., 1986, J. Comput. Phys. 62, 199.

Koplik, J., and J. R. Banavar, 1992, Science 257, 1664.

Koplik, J., and J. R. Banavar, 1993, Phys. Fluids A 5, 521.

Koplik, J., J. R. Banavar, and J. F. Willemsen, 1989, Phys. Fluids A 1, 781.

Kowalewski, T. A., 1996, Fluid Dyn. Res. 17, 121.

Ladyzhenskaya, O., 1969, The Mathematical Theory of Viscous Incompressible Flow, 2nd ed. (Gordon and Breach, New York).

Lafaurie, B., C. Nardone, R. Scardovelli, S. Zaleski, and G. Zanetti, 1994, J. Comput. Phys. 113, 134.

Lafrance, P., 1975, Phys. Fluids 18, 428.

Landau, L. D., and E. M. Lifshitz, 1984a, Fluid Mechanics (Pergamon, Oxford).

Landau, L. D., and E. M. Lifshitz, 1984b, Electrodynamics of Continuous Media (Pergamon, Oxford).

Lee, H. C., 1974, IBM J. Res. Dev. 18, 364.

Leib, S. J., and M. E. Goldstein, 1986, J. Fluid Mech. 168, 479.

Leonhard, H., 1996, "Experiments on tipstreaming," unpublished.

Li, H., T. C. Halsey, and A. Lobkovsky, 1994, Europhys. Lett. 27, 575.

Lin, S. P., and D. J. Kang, 1987, Phys. Fluids 30, 2000.

Lin, S. P., and R. D. Webb, 1994, Phys. Fluids 6, 2671.

Lister, J. R., and H. A. Stone, 1996, J. Fluid Mech. 317, 275.

Lister, J. R., H. Stone, and M. P. Brenner, 1997 unpublished.

Longuet-Higgins, M. S., and E. D. Cokelet, 1976, Proc. R. Soc. London, Ser. A 350, 1.

Magnus, G., 1855, Ann. Phys. Chem. 95, 1.

Majda, A. J., 1991, SIAM (Soc. Ind. Appl. Math.) Rev. 39, 349. Mansour, N. N., and T. S. Lundgren, 1990, Phys. Fluids A 2, 1141.

Manwell, A. R., 1971, The Hodograph Equations (Hafner, Darien, Connecticut).

Mariotte, E., 1686, Traité du mouvement des eaux et des autres corps fluides (E. Michallet, Paris). 
Marschall, E., 1985, Verh. Deutsch. Ingen. Forschungsheft 632, 13.

Matovitch, M. A., and J. R. A. Pearson, 1969, Ind. Eng. Chem. Fundamentals 8, 512.

Maxwell, J. C., 1875, in Encyclopedia Britannica, 9th edition. Reprinted 1965 in The Scientific Papers of James Clerk Maxwell, edited by W. D. Niven (Dover, New York), Vol. II, p. 541.

McCarthy, M. J., and N. A. Malloy, 1974, Chem. Eng. J. 7, 1. Merle, F., 1992, Commun. Pure Appl. Math. 45, 203.

Meseguer, J., 1983, J. Fluid Mech. 130, 123.

Meseguer, J., and A. Sanz, 1985, J. Fluid Mech. 153, 83.

Meseguer, J., A. Sanz, and J. Lopez, 1986, J. Cryst. Growth 78, 325.

Mestel, A. J., 1994, J. Fluid Mech. 274, 93.

Michael, D. H., and P. G. Williams, 1976, Proc. R. Soc. London, Ser. A 351, 117.

Middleman, S., 1995, Modeling Axisymmetric Flows (Academic, New York).

Mikami, T., R. G. Cox, and S. G. Mason, 1975, J. Multiphase Flow 2, 113.

Miksis, M. J., 1981, Phys. Fluids 24, 1967.

Moffatt, H. K., 1995, private communication.

Moore, D. W., 1983, IMA J. Appl. Math. 31, 1.

Müller, A., and S. Grossmann, 1985, Z. Naturforsch. Teil A 40, 968.

Nayfeh, A. H., 1970, Phys. Fluids 14, 841.

Nobari, M. R., Y.-J. Jan, and G. Tryggvason, 1996, Phys. Fluids $\mathbf{8}, 29$.

O $\widetilde{g}$ uz, H. N., and A. Prosperetti, 1990, J. Fluid Mech. 219, 143.

Olver, P. J., and Y. Nutku, 1988, J. Math. Phys. (N.Y.) 29, 1610.

Pantano, C., A. M. Gañ án-Calvo, and A. Barrero, 1994, J. Aerosol Sci. 25, 1065.

Papageorgiou, D. T., 1995, Phys. Fluids 7, 1529.

Pearson, J. R. A., and M. A. Matovitch, 1969, Ind. Eng. Chem. Fundamentals 8, 605.

Pelekasis, N. A., J. A. Tsamopoulos, and G. D. Manolis, 1992, J. Comput. Phys. 101, 231.

Perales, J. M., and J. Meseguer, 1992, Phys. Fluids 4, 1110.

Peregrine, D. H., 1972, in Waves on Beaches, edited by R. E. Meyer (Academic, New York), p. 95.

Peregrine, D. H., G. Shoker, and A. Symon, 1990, J. Fluid Mech. 212, 25.

Pimbley, W. T., 1976, IBM J. Res. Dev. 20, 148.

Pimbley, W. T., and H. C. Lee, 1977, IBM J. Res. Dev. 21, 21.

Plateau, J., 1843, Acad. Sci. Bruxelles Mém. 16, 3.

Plateau, J., 1849, Acad. Sci. Bruxelles Mém. 23, 5.

Plateau, J., 1857, Acad. Sci. Bruxelles Mém. 30, 3.

Plateau, J., 1873, Statique Expérimentale et Théoretique des Liquides Soumis aux Seules Forces Moléculaires (Gauthier Villars, Paris), Vol. II, p. 319.

Pomeau, Y., 1994, C. R. Acad. Sci. Paris 318, 865.

Press, W. H., S. A. Teukolski, W. T. Vetterling, and B. P. Flannery, 1992, Numerical Recipes, 2nd ed. (Cambridge University, Cambridge, England).

Pumir, A., and E. D. Siggia, 1992, Phys. Fluids A 4, 1472.

Rallison, J. M., and A. Acrivos, 1978, J. Fluid Mech. 89, 191.

Rallison, J. M., 1984, Annu. Rev. Fluid Mech. 16, 45.

Rayleigh, Lord, J. W. S., 1879a, Proc. London Math. Soc. 10, 4 (appeared in the volume of 1878).

Rayleigh, Lord, J. W. S., 1879b, Proc. R. Soc. London, Ser. A 29, 94 .
Rayleigh, Lord, J. W. S., 1882, Proc. R. Soc. London, Ser. A 34, 130.

Rayleigh, Lord, J. W. S., 1891, Nature (London) 44, 249.

Rayleigh, Lord, J. W. S., 1892, Philos. Mag. 34, 145.

Rayleigh, Lord, J. W. S., 1896, The Theory of Sound (Macmillan, London). Reprinted 1945, 2nd ed. (Dover, New York), Vol. II, p. 351.

Rayleigh, Lord, J. W. S., 1899, Philos. Mag. 48, 321.

Razumovskiǔ, N. A., 1993, Zh. Tekh. Fiz. 63, 26 [Tech. Phys. 38, 752 (1993)].

Rehage, H., and H. Hoffmann, 1991, Mol. Phys. 74, 933.

Renardy, M., 1982, SIAM (Soc. Ind. Appl. Math.) J. Math. Anal. 13, 226.

Renardy, M., 1994, J. Non-Newton. Fluid Mech. 51, 97.

Renardy, M., 1995, J. Non-Newton. Fluid Mech. 59, 267.

Reynolds, O., 1886, Philos. Trans. R. Soc. London 177, 157.

Richards, J. R., A. M. Lenhoff, and A. N. Beris, 1994, Phys.

Fluids 6, 2640.

Rutland, D. F., and G. J. Jameson, 1970, Chem. Eng. Sci. 25, 1689.

Rutland, D. F., and G. J. Jameson, 1971, J. Fluid Mech. 46, 267.

Savart, F., 1833, Annal. Chim. 53, 337, plates in Vol. 54.

Schneider, J. M., N. R. Lindblatt, C. D. Hendricks, Jr., and J.

M. Crowley, 1967, J. Appl. Phys. 38, 2599.

Schulkes, R. M. S. M., 1993, Phys. Fluids A 5, 2121.

Schulkes, R. M. S. M., 1994a, J. Fluid Mech. 261, 223.

Schulkes, R. M. S. M., 1994b, J. Fluid Mech. 278, 83.

Schultz, W. W., and S. H. Davis, 1982, J. Rheol. 26, 331.

Sedov, L. I., 1946, Prikl. Mat. Mekh. 10, 241.

Sherwood, J. D., 1981, Math. Proc. Camb. Philos. Soc. 90, 529.

Sherwood, J. D., 1984, J. Fluid Mech. 144, 281.

Sherwood, J. D., 1988, J. Fluid Mech. 188, 133.

Sherwood, J. D., 1991, J. Phys. A 24, 4047.

Shi, X. D., M. P. Brenner, and S. R. Nagel, 1994, Science 265, 157.

Shield, T. W., D. B. Bogy, and F. E. Talke, 1986, J. Comput. Phys. 67, 327.

Shipman, R. W. G., M. M. Denn, and R. Keunings, 1991, J. Non-Newton. Fluid Mech. 40, 281.

Shokoohi, F., and H. G. Elrod, 1987, J. Comput. Phys. 71, 324. Shokoohi, F., and H. G. Elrod, 1990, J. Comput. Phys. 89, 483. Spangler, C. A., J. H. Hilbing, and S. D. Heister, 1995, Phys. Fluids 7, 964.

Spiegelberg, S. H., D. C. Ables, and G. H. McKinley, 1996, J. Non-Newton. Fluid Mech. 64, 229.

Spiegelberg, S. H., S. Gaudet, and G. H. McKinley, 1994, in Extensional Deformation of Non-Newtonian Materials: Liquid Bridge Studies, 2nd Microgravity Fluid Physics Conference, Cleveland, Ohio, Vol. CP-3276 (U.S. Govt. Printing Office 1994-553-454), p. 311.

Spiegelberg, S. H., and G. H. McKinley, 1996, J. Non-Newton. Fluid Mech. 67, 49.

Sridhar, T. S., V. Tirtaatmadja, D. A. Nguyen, and R. K. Gupta, 1991, J. Non-Newton. Fluid Mech. 40, 271.

Stone, H. A., 1994, Annu. Rev. Fluid Mech. 26, 65.

Stone, H. A., B. J. Bentley, and L. G. Leal, 1986, J. Fluid Mech. 173, 131.

Stone, H. A., and M. P. Brenner, 1996, J. Fluid Mech. 318, 373. Stone, H. A., and L. G. Leal, 1989a, J. Fluid Mech. 198, 399. Stone, H. A., and L. G. Leal, 1989b, J. Fluid Mech. 206, 223. Stone, H. A., J. R. Lister, and M. P. Brenner, 1997, "Drops with conical ends in electric and magnetic fields," Harvard University preprint. 
Tate, T., 1864, Philos. Mag. 27, 176.

Taub, H. H., 1976, Phys. Fluids 19, 1124.

Taylor, G. I., 1932, Proc. R. Soc. London, Ser. A 138, 41. Taylor, G. I., 1934, Proc. R. Soc. London, Ser. A 146, 501.

Taylor, G. I., 1964, Proc. R. Soc. London, Ser. A 280, 383.

Taylor, G. I., 1966, in Proceedings of 11th International Congress of Applied Mechanics, Munich, 1964 (Springer, Heidelberg). Reprinted 1971 in The Scientific Papers of Sir Geoffry Ingram Taylor, edited by G. K. Batchelor (Cambridge University, Cambridge, England), Vol. IV.

Taylor, G. I., 1969, Proc. R. Soc. London, Ser. A 313, 453.

Ting, L., and J. B. Keller, 1990, SIAM (Soc. Ind. Appl. Math.) J. Appl. Math. 50, 1533.

Tjahjadi, M., and J. M. Ottino, 1991, J. Fluid Mech. 232, 191. Tjahjadi, M., H. A. Stone, and J. M. Ottino, 1992, J. Fluid Mech. 243, 297.

Tomotika, S., 1935, Proc. R. Soc. London, Ser. A 150, 322.

Tomotika, S., 1936, Proc. R. Soc. London, Ser. A 153, 302.

Torpey, P. A., 1988, Phys. Fluids A 1, 661.

Trouton, F. T., 1906, Proc. R. Soc. London, Ser. A 77, 426.

Tsai, W.-T., and D. K. P. Yue, 1996, Annu. Rev. Fluid Mech. 28, 249.

Tur, A. V., and V. V. Yanovsky, 1993, J. Fluid Mech. 248, 67. Van Dyke, M., 1975, Perturbation Methods in Fluid Mechanics, second Ed. (Parabolic, Stanford, California).

Vassallo, P., and N. Ashgriz, 1991, Proc. R. Soc. London, Ser. A 433, 269.
Walzel, P., 1988, "Spraying and Atomizing of Liquids," in $U l$ lmann's Encyclopedia of Industrial Chemistry, 5th Ed., Vol. B2 (VCH, Weinheim).

Wang, D. P., 1968, J. Fluid Mech. 34, 299.

Weast, R. C., 1978, Ed., Handbook of Chemistry and Physics (CRC Press, Cleveland, Ohio).

Weber, C., 1931, Z. Angew. Math. Mech. 11, 136.

Wilson, S. D. R., 1988, J. Fluid Mech. 190, 561.

Worthington, A. M., 1881, Proc. R. Soc. London, Ser. A 32, 362.

Worthington, A. M., 1908, A Study of Splashes (Longmans, London).

Yang, H. Q., 1992, Phys. Fluids A 4, 681.

Yarin, A. L., 1993, Free Liquid Jets and Films: Hydrodynamics and Rheology (Wiley, New York).

Yarin, A. L., and D. A. Weiss, 1995, J. Fluid Mech. 283, 141. Young, T., 1805, Philos. Trans. R. Soc. London 95, 65.

Youngren, G. K., and A. Acrivos, 1975, J. Fluid Mech. 69, 377. Yuen, M.-C., 1968, J. Fluid Mech. 33, 151.

Zeleny, J., 1914, Phys. Rev. 3, 69.

Zeleny, J., 1915, Proc. Camb. Philos. Soc. 18, 71.

Zeleny, J., 1917, Phys. Rev. 10, 1.

Zhang, X., and O. A. Basaran, 1995, Phys. Fluids 7, 1184.

Zhang, X., R. S. Padgett, and O. A. Basaran, 1996, J. Fluid Mech. 329, 207. 